NBER WORKING PAPER SERIES

\title{
ORGANIZATIONAL BARRIERS TO TECHNOLOGY ADOPTION: EVIDENCE FROM SOCCER-BALL PRODUCERS IN PAKISTAN
}

\author{
David Atkin \\ Azam Chaudhry \\ Shamyla Chaudry \\ Amit K. Khandelwal \\ Eric Verhoogen \\ Working Paper 21417 \\ http://www.nber.org/papers/w21417 \\ NATIONAL BUREAU OF ECONOMIC RESEARCH \\ 1050 Massachusetts Avenue \\ Cambridge, MA 02138 \\ July 2015
}

We are grateful to Tariq Raza, our project manager, and to Research Consultants (RCONS), our local survey firm, in particular Irfan Ahmad, Kashif Abid and Syed Hassan Raza Rizvi, for tireless work in carrying out the firm surveys; to Fatima Aqeel, Muhammad Haseeb Ashraf, Sabyasachi Das, Golvine de Rochambeau, Abdul Rehman Khan, Meng-han Xu, and Daniel Rappoport for excellent research assistance; to Laura Alfaro, Charles Angelucci, Simon Board, Esther Duflo, Florian Ederer, Cecilia Fieler, Dean Karlan, Navin Kartik, Asim Khwaja, Rocco Macchiavello, David McKenzie, Moritz Meyer-ter-Vehn, Ben Olken, Ralph Ossa, Anja Sautmann, Chris Udry, Johannes Van Biesebroeck, Rodrigo Wagner, Reed Walker, Chris Woodruff, Daniel $\mathrm{Xu}$ and many seminar audiences for helpful discussions; and to the International Growth Centre, especially Ijaz Nabi and Naved Hamid, for generous research support. We are particularly indebted to Annalisa Guzzini, who shares credit for the invention of the new technology described in the text, and to Naved Hamid, who first suggested we study the soccer-ball sector in Sialkot. All errors are ours. The views expressed herein are those of the authors and do not necessarily reflect the views of the National Bureau of Economic Research.

At least one co-author has disclosed a financial relationship of potential relevance for this research. Further information is available online at http://www.nber.org/papers/w21417.ack

NBER working papers are circulated for discussion and comment purposes. They have not been peer-reviewed or been subject to the review by the NBER Board of Directors that accompanies official NBER publications.

(C) 2015 by David Atkin, Azam Chaudhry, Shamyla Chaudry, Amit K. Khandelwal, and Eric Verhoogen. All rights reserved. Short sections of text, not to exceed two paragraphs, may be quoted without explicit permission provided that full credit, including (C) notice, is given to the source. 
Organizational Barriers to Technology Adoption: Evidence from Soccer-Ball Producers in

Pakistan

David Atkin, Azam Chaudhry, Shamyla Chaudry, Amit K. Khandelwal, and Eric Verhoogen

NBER Working Paper No. 21417

July 2015, Revised July 2016

JEL No. D2,L2,O1,O3

\section{ABSTRACT}

This paper studies technology adoption in a cluster of soccer-ball producers in Sialkot, Pakistan. We invented a new cutting technology that reduces waste of the primary raw material and gave the technology to a random subset of producers. Despite the clear net benefits for nearly all firms, after 15 months take-up remained puzzlingly low. We hypothesize that an important reason for the lack of adoption is a misalignment of incentives within firms: the key employees (cutters and printers) are typically paid piece rates, with no incentive to reduce waste, and the new technology slows them down, at least initially. Fearing reductions in their effective wage, employees resist adoption in various ways, including by misinforming owners about the value of the technology. To investigate this hypothesis, we implemented a second experiment among the firms that originally received the technology: we offered one cutter and one printer per firm a lump-sum payment, approximately a month's earnings, conditional on demonstrating competence in using the technology in the presence of the owner. This incentive payment, small from the point of view of the firm, had a significant positive effect on adoption. The results suggest that misalignment of incentives within firms is an important barrier to technology adoption in our setting.

David Atkin

Department of Economics, E52-550

MIT

77 Massachusetts Avenue

Cambridge, MA 02139

and NBER

atkin@mit.edu

Azam Chaudhry

Department of Economics

Lahore School of Economics

azam@lahoreschool.edu.pk

Shamyla Chaudry

Department of Economics

Lahore School of Economics

shamyla@lahoreschool.edu.pk
Amit K. Khandelwal

Graduate School of Business

Columbia University

Uris Hall 606, 3022 Broadway

New York, NY 10027

and NBER

ak2796@columbia.edu

Eric Verhoogen

Department of Economics

School of International \& Public Affairs

Columbia University

420 W. 118th Street

New York, NY 10027

and NBER

eric.verhoogen@columbia.edu 


\section{Introduction}

Observers of the process of technological diffusion have been struck by how slow it is for many technologies. ${ }^{1}$ A number of the best-known studies have focused on agriculture or medicine ${ }^{2}$ but diffusion has also been observed to be slow among large firms in manufacturing. In a classic study of major industrial technologies, Mansfield (1961) found that it took more than 10 years for half of major U.S. iron and steel firms to adopt by-product coke ovens or continuous annealing lines, technologies that were eventually adopted by all major firms. ${ }^{3}$ More recently, Bloom, Eifert, Mahajan, McKenzie, and Roberts (2013) found that many Indian textile firms are not using standard (and apparently cheap to implement) management practices that have diffused widely elsewhere. The surveys by Hall and Khan (2003) and Hall (2005) contain many more examples.

Why is adoption so slow for so many technologies? The question is key to understanding the process of economic development and growth. It is also a difficult one to study empirically, especially among manufacturing firms. It is rare to be able to observe firms' technology use directly, and rarer still to have direct measures of the costs and benefits of adoption, or of what information firms have about a given technology. As a consequence, it is difficult to distinguish between various possible explanations for low adoption rates.

In this paper, we present evidence from a cluster of soccer-ball producers in Sialkot, Pakistan, that a conflict of interest between employees and owners within firms is an important barrier to adoption. The cluster is economically significant, producing 30 million soccer balls per year, including for the largest global brands (and the 2014 World Cup). The setting has two main advantages for understanding the adoption process. The first is that the industry is populated by a substantial number of firms - 135 by our initial count — producing a relatively standardized product, using largely the same, simple production process. The technology we focus on is applicable at a large enough number of firms to conduct statistical inference. The second, and perhaps more important, advantage is that our research team, through a series of fortuitous events, discovered a useful innovation: we invented a new technology that represents, we argue, a clear increase in technical efficiency for nearly all firms in the sector. The most common soccer-ball design combines 20 hexagonal and 12 pentagonal panels. The panels are cut from rectangular sheets of an artificial leather called rexine, typically by bringing a hydraulic press down on a hand-held metal die. Our new technology, described in more detail below, is a die that increases the number of pentagons that can be cut from a rectangular sheet, by implementing the densest packing of pentagons in a plane known to mathematicians. A conservative estimate is that the new die reduces rexine cost per pentagon by 6.76 percent and total costs by approximately 1 percent - a modest reduction

\footnotetext{
${ }^{1}$ In a well-cited review article, Geroski (2000, p.604) writes: "The central feature of most discussions of technology diffusion is the apparently slow speed at which firms adopt new technologies." Perhaps the foremost economic historian of technology, Nathan Rosenberg, writes "[I]f one examines the history of the diffusion of many inventions, one cannot help being struck by two characteristics of the diffusion process: its apparent overall slowness on the one hand and the wide variations in the rates of acceptance of different inventions on the other" (Rosenberg (1972, p. 6)).

${ }^{2}$ See, for instance, Ryan and Gross (1943), Griliches (1957), Coleman and Menzel (1966), Foster and Rosenzweig (1995), and Conley and Udry (2010).

${ }^{3}$ See also the summary in Table 2 of Mansfield (1989).
} 
but a non-trivial one in an industry where mean profit margins are 8 percent. The new die requires minimal adjustments to other aspects of the production process. Importantly, we observe adoption of the new die very accurately, in contrast to studies that must infer technology adoption from changes in residual-based measures of productivity such as those reviewed in Syverson (2011).

We randomly allocated the new technology to a subset of 35 firms (which we refer to as the "tech drop" group) in May 2012. To a second group of 18 firms (the "cash drop" group) we gave cash equal to the value of the new die (US\$300), and to a third group of 79 firms (the "no drop" group) we gave nothing. We expected the technology to be adopted quickly by the tech-drop firms, and we planned to focus on spillovers to the cash-drop and no-drop firms; we are pursuing this line of inquiry in a companion project. In the first 15 months of the experiment, however, the most striking fact was how few firms had adopted, even among the tech-drop group. As of August 2013, five firms from the tech-drop group and one from the no-drop group had used the new die to produce more than 1,000 balls, our preferred measure of adoption. ${ }^{4}$ The experiences of the adopters indicated that the technology was working as expected; we were reassured, for instance, by the fact that the one no-drop adopter was one of the largest firms in the cluster, and had purchased a total of 40 dies on 15 separate occasions. Overall, however, adoption remained puzzlingly low.

In our March-April 2013 survey round, we asked non-adopters in the tech-drop group why they had not adopted. Of a large number of possible responses, the leading answer was resistance from employees. Anecdotal evidence from a number of firms also suggested that workers were resisting the new die, in part by misinforming owners about the productivity benefits of the die. Moreover, we noticed that the pay scheme of the large adopter (purchaser of the 40 dies) differed from the local norm: while more than 90 percent of firms pay a pure piece rate, it pays a fixed monthly salary plus a performance bonus.

The qualitative evidence led us to hypothesize that a misalignment of incentives within the firm is an important reason for the lack of adoption. The new die slows cutters down, certainly in the initial period when they are learning how to use it, and possibly in the longer run. ${ }^{5}$ If the piece rate is not adjusted, the cutters' effective wage falls. Unless owners modify the payment scheme, the benefits of using the new technology accrue to owners and the costs are borne by the cutters. Realizing this, the workers resist adoption, including by misinforming owners about the value of the technology. We formalize this intuition in a simple model of strategic communication between an imperfectly informed principal (the owner) and a perfectly informed agent (the cutter). When principals are limited to standard piece-rate contracts that must be set ex-ante, there is an equilibrium in which the agent seeks to discourage the principal from adopting a technology like ours and influences the principal not to adopt. ${ }^{6}$ A simple modification to the labor contract, conditioning

\footnotetext{
${ }^{4}$ In this introduction, we use our "liberal" measure of adoption; see Section 3 below for a discussion of our "liberal" and "conservative" adoption measures.

${ }^{5}$ Our data suggest that the long-run speed is only a little slower than for the existing die, although the workers did not know this prior to adoption and may plausibly have been worried that they would be substantially slower in the long run as well as the short.

${ }^{6}$ If we assume, following Crawford and Sobel (1982), that players are able to coordinate on the "most informative" equilibrium in every cheap-talk subgame, then this equilibrium is unique.
} 
the wage contract on marginal cost, an ex-post-revealed characteristic of the technology, induces the agent to encourage adoption of the technology and the principal to adopt it.

To investigate the misalignment-of-incentives hypothesis, we implemented a second experiment. In September 2013, we randomly divided the set of 31 tech-drop firms that were still in business into two subgroups, a treatment subgroup (which we call Group A) and a control subgroup (Group B). To Group B, we simply gave a reminder about the benefits of the die and an offer of another demonstration of the cutting pattern. To Group A, we gave the reminder but also explained the issue of misaligned incentives to the owner and offered an incentive-payment treatment: we offered to pay one cutter and one printer a lump-sum bonus roughly equivalent to a month's earnings (US $\$ 150$ and US\$120, respectively), conditional on demonstrating competence with the new technology in the presence of the owner. This bonus was designed to mimic (as closely as possible, given firms' reluctance to participate) the "conditional" wage contracts we model in the theory. (We also paid printers a bonus because the new die requires a slight modification to the printing process, and printers face a similar but weaker disincentive to adopt.) The one-time bonus payments were small relative both to revenues from soccer-ball sales for the firms, approximately US $\$ 57,000$ per month at the median, and to the variable-cost reductions from adopting of our technology, approximately US\$413 per month at the median.

Fifteen firms were assigned to Group A and 16 to Group B. Of the 13 Group A firms that had not already adopted the new die, 8 accepted the incentive-payment intervention, and five adopted the new die within six months of the second experiment. Of the 13 Group B firms that had not already adopted the new die, none adopted within six months of the experiment and one adopted in the next six-month period. Although the sample size is small, we find a positive, statistically significant effect of the incentive intervention on adoption, with the probability of adoption increasing by 0.27-0.32 from a baseline adoption rate of 0.16 in our intent-to-treat specifications. Our results remain significant when using permutation tests that are robust to small sample sizes. The fact that such small payments had a significant effect on adoption suggests that the misalignment of incentives is indeed an important barrier to adoption in this setting.

After the second experiment, we asked owners and their employees a number of survey questions about wage-setting and communication within the firm, and the results are supportive of the hypotheses that piece-rate contracts are sticky and that cutters have misinformed owners about the technology. We argue that the results do not support a leading alternative hypothesis, that our second experiment mechanically induced adoption by subsidizing the fixed costs of adoption, because it cannot quantitatively explain both low initial adoption rates and an increase in adoption of the magnitude we find in response to the second experiment. We also argue against the hypothesis that the second experiment merely increased the salience of the technology to owners, and that this alone led to increased adoption.

A natural question is why the firms themselves did not adjust their payment schemes to incentivize their employees to adopt the technology. Our model suggests two possible explanations. The first is that owners simply did not realize that such an alternative payment scheme was possible or 
desirable, just as the technical innovation had not occurred to them. The second is that there was a transaction cost involved in changing payment schemes that exceeded the expected benefits in this case. In the end, the two hypotheses have similar observable implications and are difficult to distinguish empirically. The important point is that many firms did not in fact adjust the payment scheme and as a consequence there was scope for our modest payment intervention to have an effect on adoption.

To our knowledge, this is the first study to randomly allocate a new technology to manufacturing firms to examine the adoption process. ${ }^{7}$ But the paper is related to several different literatures. There is a long literature, largely descriptive, on how conflicts of interest in the workplace may affect productivity. One strand, dating back at least to the work of Taylor $(1896,1911)$ on scientific management, and including classic studies by Mathewson (1931), Roy (1952), Edwards (1979) and Clawson (1980), has focused on how employees in piece-rate systems may conceal information about how quickly they are capable of working or about productivity improvements they have discovered. ${ }^{8}$ A distinctive aspect of our study, relative to this strand, is the focus on workers dissuading owners from using a technology from outside the firm. Another strand of literature, much of it focusing on historical cases, has emphasized worker resistance to the adoption of such externally-developed technologies (Lazonick, 1979, 1990; Mokyr, 1990). But the most commonly cited cases are of resistance to labor-saving technologies by groups of skilled artisans whose skills were in danger of being rendered obsolete. By contrast, our technology is labor-using: the new die simply slows cutters down while they are learning to use it, and possibly in the longer run; we would expect demand for skilled cutters to increase marginally. There appears to have been little previous empirical research on worker resistance to such technologies.

A number of other explanations for slow technology adoption by manufacturing firms have been advanced. Bloom and Van Reenen (2007, 2010) and Bloom et al. (2013) suggest that a lack of product-market competition may be responsible for the failure to adopt beneficial management practices. Rosenberg (1982), David (1990), Bresnahan and Trajtenberg (1995) and others have emphasized that new technologies often require changes in complementary technologies, which take time to implement. In our setting, firms sell almost all output on international export markets that appear to be quite competitive, and our technology requires extremely modest changes to other aspects of production, so these common explanations do not appear to be directly applicable.

There is an active literature in development economics on technology adoption in agriculture, where measures of technology use are more readily available than in manufacturing (e.g. Foster and Rosenzweig (1995), Munshi (2004), Bandiera and Rasul (2006), Conley and Udry (2010), Duflo, Kremer, and Robinson (2011), Suri (2011), Hanna, Mullainathan, and Schwartzstein (2014), BenYishay and Mobarak (2014), Beaman, Ben-Yishay, Magruder, and Mobarak (2015), Emerick, de Janvry, Sadoulet, and Dar (2016)). In addition to being important for development in their

\footnotetext{
${ }^{7}$ A recent study by Hardy and McCasland (2016), which appeared after our paper was widely circulated, randomly allocated a weaving technology to textile producers in Ghana, but focuses on diffusion rather than barriers to adoption.

${ }^{8} \mathrm{~A}$ more recent precursor is a case study by Freeman and Kleiner (2005), who provide descriptive evidence that an American shoe company's shift away from piece rates helped it to increase productivity.
} 
own right, manufacturing firms raise issues of organizational conflict that do not arise when the decision-makers are individual smallholder farmers. Also, risk arguably plays a less important role among manufacturing firms, both because there is a lower degree of production risk (making inference about the benefits of a technology more straightforward) and because factory owners are typically richer and presumably less risk-averse than smallholder farmers. ${ }^{9}$

Our paper is also related to a small but growing literature on field experiments in firms, including the experiments with fruit-pickers by Bandiera, Barankay, and Rasul (2005, 2007, 2009) and with microenterprises by de Mel, McKenzie, and Woodruff (2008). ${ }^{10}$ The Bloom et al. (2013) study, mentioned above, randomly allocated consulting services among Indian textile plants and argues that this generated exogenous variation in management practices (arguably a form of technology) which can be used to estimate the effect of those practices on plant outcomes. The authors suggest that "informational constraints" are an important factor leading firms not to adopt simple, apparently beneficial, practices that are widespread elsewhere. Our study investigates how conflicts of interest within firms can impede the flow of information to managers and provides a possible micro-foundation for such informational constraints. ${ }^{11}$

The theoretical model we develop combines ideas from the literature on strategic communication following Crawford and Sobel (1982) and the voluminous literature on principal-agent models of the employment relationship (reviewed for instance by Lazear and Oyer (2013) and Gibbons and Roberts (2013)). Gibbons (1987) formalizes the argument that (assuming employers cannot commit to future contracts) workers paid piece rates may hide information about labor-saving productivity improvements from their employers, to prevent employers from reducing rates. ${ }^{12}$ Carmichael and MacLeod (2000) explore the contexts in which firms will commit to fixing piece rates in order to alleviate these "ratchet" effects. Holmstrom and Milgrom (1991) show that high-powered incentives such as piece rates may induce employees to focus too much on the incentivized task to the detriment of other tasks, which could include reporting accurately on the value of a technology. Our study supports the argument of Milgrom and Roberts (1995) that piece rates may need to be combined with other incentives (in our case higher pay conditional on adopting the new technology) in order to achieve high performance. Perhaps the closest theoretical antecedent is Dow and Perotti (2013), in which losers within the firm may block the adoption of a new technology

\footnotetext{
${ }^{9}$ Also related are recent papers on adoption of health technologies in the presence of externalities (Miguel and Kremer, 2004; Cohen and Dupas, 2010; Dupas, 2014), on adoption of change-holding behavior by Kenyan retail micro-enterprises (Beaman, Magruder, and Robinson, 2014), and on adoption of energy-efficient technologies by households and firms in the US (Anderson and Newell, 2004; Fowlie, Greenstone, and Wolfram, 2015a,b). Again, organizational conflict does not appear to play an important role in these settings.

${ }^{10}$ See Bandiera, Barankay, and Rasul (2011) for a review of the literature on field experiments in firms.

${ }^{11}$ Our study is also related to the "insider econometrics" literature reviewed by Ichniowski and Shaw (2013), which focuses on relationships between management practices and productivity, typically in a cross-sectional context, and generally does not focus on technology adoption. A recent experimental study by Khan, Khwaja, and Olken (2016) of tax collectors in Pakistan focuses on a related issue: the effect of altering wage contracts on employee performance.

${ }^{12}$ Lazear (1986) also notes that workers under a piece-rate scheme may have an incentive to restrict output to avoid such a ratchet effect, but argues that (assuming employers can commit to future contracts) this can be counteracted by a suitable set of piece rates in all periods. The key difference between Lazear (1986) and Gibbons (1987) is the assumption about the employer's ability to commit.
} 
because it reduces their share of quasi-rents (but do not communicate strategically). ${ }^{13}$ Garicano and Rayo (2016) review a number of models of "organizational failures" that may lead firms not to adopt surplus-enhancing innovations. We view our model primarily as an application of insights from the organizational-economics literature to our setting, although we are not aware of a previous theoretical treatment of the specific idea that workers on piece-rate contracts may dissuade owners from adopting surplus-enhancing technologies from outside the firm. Our main contribution is empirical: while the organizational-economics literature has had to rely almost entirely on case studies for supporting evidence on organizational failures, we are able to document - and explore the reasons for — such a failure in an experimental setting.

The paper is organized as follows. Section 2 provides background on the Sialkot cluster and describes the new cutting technology. Section 3 describes our surveys and presents summary statistics. Section 4 details the roll-out of the new technology and documents rates of early adoption. Section 5 discusses qualitative evidence on organizational barriers and summarizes our model of strategic communication in a principal-agent context, the full exposition of which has been relegated to Appendix B. Section 6 describes the incentive-payment experiment and evaluates the results. Section 7 presents additional evidence on the hypothesized theoretical mechanisms. Section 8 considers the leading alternative interpretations of our findings, and Section 9 concludes.

\section{Background and Description of the New Technology}

\subsection{The industry}

Sialkot is a city of 1.6 million people in the province of Punjab, Pakistan. The soccer-ball cluster dates to British colonial rule. In 1889, a British sergeant asked a Sialkoti saddle-maker to repair a damaged ball, and then to make a new ball made from scratch. The industry expanded through spinoffs and new entrants. ${ }^{14}$ By the 1970s, the city had become a center of offshore production for many European soccer-ball companies. In 1982, a firm in Sialkot manufactured the FIFA World Cup ball for the first time. Virtually all of Pakistan's soccer ball production is concentrated in Sialkot and exported to foreign markets. While in recent years the industry has faced increasing competition from East Asian, especially Chinese, suppliers, ${ }^{15}$ Sialkot remains the major source for the world's hand-stitched soccer balls. It provided, for example, the hand-stitched balls used in the 2012 Olympic Games. ${ }^{16}$

To the best of our knowledge, there were 135 manufacturing firms producing soccer balls in Sialkot as of November 2011. The firms themselves employ approximately 12,000 workers, and outsourced employment of stitchers in stitching centers and households is estimated to be more

\footnotetext{
${ }^{13}$ This idea has parallels in the work of Marglin (1974) and Stole and Zwiebel (1996), who argue that owners may prefer technically less-efficient technologies that shift the distribution of rents in their favor. Also related, with very different emphases, are Dearden, Ickes, and Samuelson (1990), Aghion and Tirole (1997), Dessein (2002) and Krishna and Morgan (2008).

${ }^{14}$ The history of the industry is reviewed in more detail in Atkin et al. (2016).

${ }^{15}$ The evolution of soccer-ball imports to the U.S. (which tracks soccer balls specifically, unlike other major importers) is shown in Figure A.2.

${ }^{16}$ The ball for the 2014 World Cup, also produced in Sialkot, used a new thermo-molding technology.
} 
than twice that number (Khan, Munir, and Willmott, 2007). The largest firms have hundreds of employees and produce for international brands such as Nike and Adidas, among others. These firms manufacture both high-quality "match" and medium-quality "training" balls, often with a brand or team logo, as well as lower quality "promotional" balls, often with an advertiser's logo. The remaining producers are small- and medium-size firms (the median firm size is 25 employees) which typically produce promotional balls either for clients they meet through industry fairs and online markets or under subcontract to larger firms.

\subsection{The production process}

Before presenting our new technology, we briefly explain the standard production process. As mentioned above, most soccer balls (approximately 90 percent in our sample) are of a standard design combining 20 hexagons and 12 pentagons (see Figure A.1), often referred to as the "buckyball" design. ${ }^{17}$ There are four stages of production. In the first stage, shown in Figure A.3, layers of cloth (cotton and/or polyester) are glued to an artificial leather called rexine using a latex-based adhesive, to form what is called a laminated rexine sheet (henceforth "laminated rexine"). The rexine, cloth and latex are the most expensive inputs to production, together accounting for approximately 46 percent of the total cost of each soccer ball (or more if higher-quality rexine, which tends to be imported, is used). In the second stage, shown in Figure A.4, a skilled cutter uses a metal die and a hydraulic press to cut the hexagonal and pentagonal panels from the laminated rexine. The cutter positions the die by hand before activating the press with a foot-pedal. He then slides the rexine along and places the die again to make the next cut. ${ }^{18}$ In the third stage, shown in Figure A.5, logos or other insignia are printed on the panels. This requires a "screen," held in a wooden frame, that allows ink to pass through to create the desired design. Typically the dies cut pairs of hexagons or pentagons, making an indentation between them but leaving them attached to be printed as a pair, using one swipe of ink. In the fourth stage, shown in Figure A.6, the panels are stitched together around an inflatable bladder. Unlike the previous three stages, this stage is often outsourced to specialized stitching centers or stitchers' homes. This stage is the most labor-intensive part of the production process, accounting for approximately 71 percent of total labor costs.

The production process is remarkably similar across the range of firms in Sialkot. A few larger firms have automated the cutting process, cutting half or full laminated rexine sheets at once, or attaching a die to a press that moves on its own, but even these firms typically continue to do handcutting for a substantial share of their production. A few firms in the cluster have implemented machine-stitching, but this has no implications for the first three stages of production.

Prior to our study, the most commonly used dies cut two panels at a time with the panels sharing an entire edge (Figure 1). Hexagons tessellate (i.e. completely cover a plane), and experienced cutters are able to cut with a small amount of waste - approximately 8 percent of a laminated rexine sheet, mostly around the edges. (See the laminated rexine "net" remaining after cutting hexagons in Figure 2.) By contrast, pentagons do not tessellate, and using the traditional

\footnotetext{
${ }^{17}$ The buckyball design is based on a geodesic dome designed by R. Buckminster Fuller.

${ }^{18}$ We use the male pronoun because all of the cutters, printers and owners we have encountered have been men.
} 
two-pentagon die even experienced cutters typically waste 20-24 percent of the laminated rexine sheet (Figure 3). The leftover laminated rexine has little value; typically it is sold to brickmakers who burn it to fire their kilns.

\subsection{The innovation}

In June 2011, as we were first exploring the possibility of studying the soccer-ball sector, we sought out a consultant who could recommend a beneficial new technique or practice that had not yet diffused in the industry. We found a Pakistan-based consultant who appears to have been responsible for introducing the existing two-hexagon and two-pentagon dies many years ago. (Previously, firms had used single-panel dies.) We offered the consultant US $\$ 4,125$ to develop a cost-saving innovation for us. The consultant spent several days in Sialkot but was unable to improve on the existing technology. After this setback, a co-author on this project, Eric Verhoogen, happened to watch a YouTube video of a Chinese firm producing the Adidas "Jabulani" thermo-molded soccer ball used in the 2010 FIFA World Cup. The video showed an automated press cutting pentagons for an interior lining of the Jabulani ball, using a pattern different from the one we knew was being used in Sialkot (Figure A.7). Based on the pattern in the video, Verhoogen and Annalisa Guzzini, an architect (who is also his wife), developed a blueprint for a four-pentagon die (Figure 4). ${ }^{19}$ Through an intermediary, we contracted with a diemaker in Sialkot to produce the die (Figure A.8). It was only after we had received the first die and piloted it with a firm in Sialkot that we discovered that the cutting pattern is well known to mathematicians. The pattern appeared in a 1990 paper in Discrete $\&$ Computational Geometry (Kuperberg and Kuperberg, 1990). ${ }^{20}$ It also appears, conveniently enough, on the Wikipedia "Pentagon" page (Figure A.9).

The pentagons in the new die are offset, with the two leftmost pentagons sharing half an edge, rather than a full edge. For this reason, we refer to the new die as the "offset" die, and treat other dies with pentagons sharing half an edge as variations on our technology. A two-pentagon variant of our design can be made using the specifications in the blueprint (with the two leftmost and two rightmost pentagons in the blueprint on the right side of Figure 4 cut separately). This version is easier to maneuver with one hand and can be used with the same cutting rhythm as the traditional two-pentagon die. It is the version that has proven more popular with firms.

\footnotetext{
${ }^{19}$ One might wonder whether firms in Sialkot also observed the production process in the Chinese firm producing for Adidas, since it was so easy for us to do so. We found one owner, of one of the larger firms in Sialkot, who said that he had been to China and observed the offset cutting pattern (illustrated in the left panel in Figure 4) and was planning to implement it on a new large cutting press to cut half a laminated rexine sheet at once, a process known as "table cutting". As of May 2012, he had not yet implemented the new pattern, however, and he had not developed a hand-held offset die. It is also important to note that two of the largest firms in Sialkot have not allowed us to see their production processes. As these two firms are known to produce for Adidas, we suspect that they were aware of the offset cutting pattern before we arrived. What is clear, however, is that neither the offset cutting pattern nor the offset die were in any other firm we visited as of the beginning of our experiment in May 2012.

${ }^{20}$ The cutting pattern represents the densest known packing of regular pentagons into a plane. Kuperberg and Kuperberg (1990) conjecture that the pattern represents the densest possible packing, but this is not a theorem.
} 


\subsection{Benefits and costs}

In order to quantify the various components of benefits and costs of using the offset die, we draw on several rounds of survey data that we describe in more detail in Section 3 below. We start by comparing the typical number of pentagons per sheet using the traditional die with the number using the offset die. The dimensions of pentagons and hexagons vary slightly across orders, even for balls of a given official size (e.g. size 5, the standard size for adults), depending on the thickness and quality of the laminated rexine sheet used. The most commonly used pentagons have edge-length $43.5 \mathrm{~mm}, 43.75 \mathrm{~mm}, 44 \mathrm{~mm}$ or $44.25 \mathrm{~mm}$ after stitching. The first two columns of Table 1 report the means and standard deviations of the numbers of pentagons per sheet for each size, using a standard (39 in. by 54 in.) sheet of laminated rexine. Column 1 uses information from owner self-reports; we elicited the information in more than one round, and here we pool observations across rounds. Column 2 reports direct observations by our survey team, during the implementation of our first experiment. In the fifth row, we have multiplied the raw measures by the ratio of means for size $44 \mathrm{~mm}$ and the corresponding size; the rescaled measure provides an estimate of the number of pentagons per sheet the firm would obtain using a $44 \mathrm{~mm}$ die. The owner reports and direct observations correspond reasonably closely, with owners slightly overestimating pentagons per sheet relative to our direct observations. Both measures suggest that cutters obtain approximately 250 pentagons per sheet using the traditional die.

Using the offset die and cutting $44 \mathrm{~mm}$ pentagons, it is possible to achieve 272 pentagons per sheet, as illustrated in Figure $4 .{ }^{21}$ For $43.5 \mathrm{~mm}$ pentagons, it is possible to achieve 280 pentagons. Columns 3-4 of Table 1 report the means and standard deviations of pentagons per sheet using the offset die. As discussed in more detail below, relatively few firms have adopted the offset die, and therefore we have few observations. But even with this caveat, we can say with a high level of confidence that more pentagons can be obtained per sheet using the offset die. The directly observed mean is 275.4 , and the standard errors indicate that difference from the mean for the traditional die (either owner reports or direct observations) is significant at greater than the 99 percent level.

In order to convert these figures into cost savings we need to know the proportion of costs that materials and cutting labor account for. Table A.1 provides a cost breakdown for a promotional ball obtained from our baseline survey. ${ }^{22}$ The table shows that the laminated rexine (rexine plus cotton/polyester plus latex) accounts for roughly half of the unit cost of production: 46 percent on average. The inflatable bladder is the second most important material input, accounting for 21 percent of the unit cost. Labor of all types accounts for 28 percent, but labor for cutting makes up less than 1 percent of the unit cost. In the second column, we report the input cost in rupees; the mean cost of a two-layer promotional ball is Rs 211 (US\$2.11). ${ }^{23}$

\footnotetext{
${ }^{21}$ If a cutter reduces the margin between cuts, or if the laminated rexine sheet is slightly larger than 39 in. by 54 in., it is possible to cut more than 272 pentagons with a size $44 \mathrm{~mm}$ die.

${ }^{22}$ In the baseline survey, firms were asked for a cost breakdown of a size- 5 promotional ball with two layers (one cotton and one polyester), the rexine they most commonly use on a two-layer size- 5 promotional ball, a glue comprised of 50 percent latex and 50 percent chemical substitute (a cheaper alternative), and a 60-65 gram inflatable latex bladder.

${ }^{23}$ The exchange rate has varied from $90 \mathrm{Rs} / \mathrm{US} \$$ to $105 \mathrm{Rs} / \mathrm{US} \$$ over the period of the study. To make calculations easy, we will use an exchange rate of $100 \mathrm{Rs} / \mathrm{US} \$$ throughout the paper.
} 
The cost savings from the offset die vary across firms, depending in part on the type of rexine used and the number of layers of cloth glued to it, which themselves depend on a firm's mix of promotional, training and match balls. In Table 2, we present estimates of the distribution of the benefits and costs of adopting the offset die for firms. Not all firms were willing to provide a cost breakdown by input in the baseline survey, and only a subset of firms have adopted the offset die. To compute the distributions, we adopt a hot-deck imputation procedure that replaces a firm's missing value for a particular cost component with a draw from the empirical distribution (i.e. non-missing values) within the firm's size stratum. ${ }^{24}$ Following Andridge and Little (2010), we bootstrap this entire procedure 1,000 times and calculate the quantiles and mean of each variable in each iteration, with the table reporting the mean and standard deviation for each statistic over the 1,000 iterations.

In Row 1 of Table 2, we report the distribution of the percentage reduction in the cost of laminated rexine used to cut pentagons when moving to the offset die. The pentagon cost reduction is 6.76 percent at the median and ranges from 4.24 percent at the $10^{\text {th }}$ percentile to 10.15 percent at the $90^{t h}$ percentile. Combining these figures with the laminated rexine share of total unit costs (which has the distribution reported in Row 2) and multiplying by 33 percent (the share of pentagons in total laminated rexine costs, since a standard ball uses more hexagons than pentagons and the hexagons are bigger) yields the percentage reduction in variable material costs reported in Row 3. The reduction in variable material costs is 0.98 percent at the median and ranges from 0.59 percent at the $10^{\text {th }}$ percentile to 1.62 percent at the $90^{\text {th }}$ percentile. ${ }^{25}$

The offset die requires cutters to be more careful in the placement of the die when cutting, at least while they are learning how to use it. A conservative estimate of the increase in labor time for cutters (for the preferred two-pentagon variant) is 100 percent. ${ }^{26}$ (In Section 6.2 below we discuss why the 100 percent number is very conservative.) If firms compensate workers for this extra labor time, labor costs will increase. The fourth row of Table 2 reports the distribution of the cutter's wage as a share of unit costs across firms. As noted earlier, the cutter's share of cost is quite low. ${ }^{27}$ Multiplying the cutter share by 33 percent (assuming that pentagons take up one third of cutting time, equivalent to their share of laminated rexine cost) and then by 100 percent (our conservative estimate of the increase in labor time) yields the percentage increase in variable labor costs from

\footnotetext{
${ }^{24}$ As discussed below, in our first experiment firms were stratified according to total monthly output (measured in number of balls) at baseline. One stratum (the "late-responder" stratum) is composed of firms that did not respond to the baseline survey. Because information on laminated rexine shares was collected only at baseline, we draw laminated rexine shares for late responders from the empirical distribution that pools the other strata. (We do not pool for the other variables, for which we have information on the late responders from later rounds.)

${ }^{25}$ Note that because a firm at a given percentile of the distribution of rexine cost reductions is not necessarily the same as the firm at that percentile of the distribution of rexine as a share of cost, the numbers should not be multiplied across rows for a particular percentile.

${ }^{26}$ As noted above, the two-pentagon version of the offset die has proven more popular with firms. As discussed in more detail in Section 4 below, we offered firms the ability to trade in the four-pentagon die, and all firms that traded in requested the less-expensive two-pentagon version. This suggests that any potential speed benefits from cutting four pentagons at a time with the four-pentagon die are negated by the fact it is heavier and so more difficult to maneuver.

${ }^{27}$ The cutter wage as a share of costs reported here is lower than in Table A.1 because that table reports input components as a share of the cost of a promotional ball. In Table 2, we explicitly account for firms' product mixes across promotional, training and match balls. To get the firm's average ball cost, we divide its reported price by one plus its reported profit margin for each ball type and then construct a weighted-average unit cost using output weights for each type. The cutter share of cost is calculated as the per-ball payment divided by this weighted-average unit cost.
} 
adopting the offset die if the firms were to compensate workers (Row 5). Although the offset die is slower and requires more care to use, our surveys suggest that firms were not concerned that adoption would lead them to miss more deadlines or increase defect rates. ${ }^{28}$

Although the proportional increase in cutting time is potentially large, the cutter's share of costs is sufficiently low that the variable labor cost increase is very small. Row 6 reports the net variable cost reduction, the difference between the variable materials cost reduction and the variable labor cost increase. The net variable cost reduction is 0.82 percent at the median, and ranges from 0.42 percent at the $10^{\text {th }}$ percentile to 1.47 percent at the $90^{\text {th }}$ percentile. Although these numbers are small in absolute terms, the cost reductions are not trivial given the low profit margins in the industry: 8.39 percent at the median and 8.42 percent at the mean. ${ }^{29}$ Row 7 shows the ratio of the net variable cost reductions to average profits; the mean and median ratios are 13.07 percent and 10.63 percent, respectively. If we multiply the net variable cost reduction by total monthly output, we obtain the total monthly savings, in rupees, from adopting the offset die (Row 8). The large variation in output across firms induces a high degree of heterogeneity in total monthly cost savings. The mean and median monthly cost savings are Rs 137,770 (US\$273) and Rs 41,350 (US\$413), respectively, and savings range from Rs 3,660 (US\$37) at the $10^{\text {th }}$ percentile to Rs 397,950 (US\$3,980) at the $90^{\text {th }}$ percentile.

These reductions in variable cost must be compared with the fixed costs of adopting the offset die. There are a number of such costs, but they are modest in monetary terms. The market price for a two-pentagon offset die is approximately Rs $10,000(\$ 100) \cdot{ }^{30}$ As we explain below, we paid this fixed cost for the firms in the tech-drop group. The offset die requires few changes to other aspects of the production process, since the pentagons that it cuts are identical to the pentagons cut by the traditional die, but there are two adjustments that many firms make, related to the fact that pairs of pentagons cut by the offset die remain attached in a different way (i.e. sharing half an edge) than those cut by the traditional die. First, for balls with printed pentagons the printing screens must be re-designed and re-made to match the offset pattern. Designers typically charge Rs 600 (US\$6) for a new design; for the minority of firms that do not have in-house screenmaking capabilities, a new screen costs Rs 200 (US\$2) from an outside screenmaker. (New screens must in any case be made for any new order but we include them to be conservative.) Second, some firms use a "combing" machine, a device to enlarge the holes at the edges of panels made by the cutting die to further facilitate sewing. These machines also use dies. A two-pentagon combing die that

\footnotetext{
${ }^{28}$ After the endline survey, we conducted a short survey on firms in Experiment 2 (see Section 6) to collect information on deadlines and defect rates. As shown in Panel A of Appendix Table A.2, defect rates for the traditional and offset dies (reported by adopters) are virtually identical. Moreover, firms did not appear concerned about missing orders prior to adopting the die, nor did any adopter report missing an order deadline because the offset die was too slow. The table further reveals that the cutting stage is not a bottleneck in the production process (Panel B of Table A.2). At full capacity, the median firm only requires 20 days a month of cutting. Firms easily adjust to reach full capacity, or to meet tight deadlines, by expanding both the number of cutters and the hours each cutter works. Moreover, most firms can find an additional cutter within a day or less. Taken together, this evidence indicates that firms were not concerned about missing deadlines if they adopted the offset die.

${ }^{29}$ The firm's profit margin is a weighted average of its reported profit margin on promotional, training and match balls where the weights are the share of each ball type in total production.

${ }^{30}$ We were initially charged Rs 30,000 (US\$300) for a four-piece die.
} 
works with pentagons cut by the two-pentagon offset die costs approximately Rs 10,000 (US\$100). For both printing and combing, it is always possible to cut and work with separate pentagons, but there is a speed benefit to keeping the pairs of pentagons attached. Adding together the cost of the die, the cost of a new screen design and screen, and a new combing die (that not all firms use), a conservative estimate of total fixed costs of adoption is Rs 20,800 (US\$208).

A common way for firms to make calculations about the desirability of adoption is to use a rule of thumb (or "hurdle") for the length of time required to recoup the fixed costs of adoption (the "payback period"). Reviewing a variety of studies from the U.S. and U.K., Lefley (1996) reports that the "hurdles" vary from 2-4 years, with the mean at approximately 3 years. ${ }^{31}$ The final two rows of Table 2 report the distribution of the number of days needed to recover the fixed costs of adoption detailed above. For this calculation, we calculate output per cutter per month and hence the cost savings per cutter per month (Row 9). Dividing our conservative estimate of per-cutter fixed costs (assuming that each cutter needs his own offset die and combing die) by the cost savings per cutter gives the number of days needed to recoup the fixed costs, reported in Row 10. The median firm can recover all fixed costs within 43 days; the payback period ranges from 10 days at the $10^{\text {th }}$ percentile to 248 days at the $90^{\text {th }}$ percentile (which corresponds to firms that produce very few balls). The final row reports the distribution of days to recover fixed costs that exclude the cost of purchasing the die; this row is relevant for the tech-drop firms, to which we gave dies at no cost. In this scenario, the median time to recover fixed costs is only 22 days, and three-quarters of firms can recover the fixed costs within eight weeks. In short, the available evidence indicates that for almost all firms, assuming that they are not extraordinarily myopic, there are clear net benefits to adopting the offset die.

\section{$3 \quad$ Data and Summary Statistics}

Between September and November 2011, we conducted a listing exercise of soccer-ball producers within Sialkot. We found 157 producers that we believed were active in the sense that they had produced soccer balls in the previous 12 months and cut their own laminated rexine. Of the 157 firms on our initial list, we subsequently discovered that 22 were not active by our definition. Of the remaining 135 firms, 3 served as pilot firms for testing our technology.

We carried out a baseline survey between January and June 2012. Of the 132 active non-pilot firms, 85 answered the survey; we refer to them as the "initial responder" sample. The low response rate was in part due to negative experiences with previous surveyors. ${ }^{32}$ In subsequent survey rounds our reputation in Sialkot improved and we were able to collect information from an additional 31 of the 47 non-responding producers (the "late responder" sample), to bring the total number of responders to 116. The baseline survey collected firm and owner characteristics, standard performance variables (e.g. output, employment, prices, product mix and inputs) and information about firms' networks (supplier, family, employee and business networks). To date, we have conducted seven

\footnotetext{
${ }^{31}$ Using data from energy-efficiency audits in the U.S., Anderson and Newell (2004) infer that firms are using hurdles of 1-2 years.

${ }^{32}$ In the mid-1990s, there was a child-labor scandal in the industry in Sialkot. Firm owners were initially quite distrustful of us in part for that reason.
} 
subsequent survey rounds: July 2012, October 2012, January 2013, March-April 2013, SeptemberNovember 2013, January-March 2014, and October-December 2014. The follow-up surveys have again collected information on the various performance measures as well as information pertinent to the adoption of the new cutting technology. In tech-drop firms, we have explicitly asked about usage of the offset die. For the other groups, we have sought to determine whether firms are using the offset die without explicitly mentioning the offset die, by probing indirectly about changes in the factory. In addition, we have obtained reports of sales of the offset dies from the six diemakers operating in Sialkot. We have detailed information on the dates firms have ordered offset dies from the diemakers, the dates the diemakers have delivered the dies, and the numbers of offset dies purchased. Based on this information, we believe that we have complete knowledge of offset dies purchased in Sialkot, even by firms that have never responded to any of our surveys.

We face three important choices about how to measure adoption. One is whether to impose a lower bound on the number of balls produced with the offset die. Several firms have reported that they have experimented with the die but have not actually used it for a client's order. To be conservative, we have chosen not to count such firms as adopters. Our preferred measure of adoption requires that firms have produced at least 1,000 balls with the offset die. The measure is not particularly sensitive to the lower bound; we perform robustness checks using different cutoffs in Section 6.2 below. (See footnote 61.) A second decision we face is how to deal with the volatility of orders and output in the industry. Many of the smaller and medium-sized firms have large orders some months and no orders in others. In addition, a particular offset die may not be useful on all orders. Ball designs and pentagon/hexagon sizes requested by clients vary, and clients have been known to request that firms use exactly the same dies as on previous orders. For these reasons, we consider the number of balls ever produced (rather than produced in the last month) with the offset die when constructing the adoption measure. The third choice we face is whether to use information we gained from firms outside of the formal survey process. Because we have been concerned about recall bias over periods of more than one month, our surveys have asked firms about their production in the previous month. However, our project team has been in regular, ongoing contact with firms, including between survey rounds, and has submitted field notes that summarize these interactions. In one case in particular, the firm told our enumerators that it had produced more than 1,000 balls using the offset die, but that order did not happen to fall in the month preceding a survey wave. To address this issue, we construct two measures of adoption: a "conservative" measure that classifies adoption using only survey data, and a "liberal" measure that classifies adoption using both survey data and field reports. Our preferred measure uses the liberal definition because it incorporates all the information we have regarding firms' activities, but we report results for both measures.

Table 3 presents summary statistics on various firm characteristics, including means and values at several quantiles. Panel A reports statistics for the sample of 85 initial responders and Panel B for the full sample that also includes the 31 late responders. Because the late responders did not respond to the baseline, we have a smaller set of variables for the full sample. As firms' responses are often noisy, where possible we have taken within-firm averages across all survey rounds for which 
we have responses (indicated by "avg ..." at the beginning of variable names in the table). Focusing on the initial-responder sample where we have more complete data, a number of facts are worth emphasizing. The median firm is medium-size (18.3 employees, producing 10,000 balls/month) but there are also some very large firms (the highest reported employment and production are 1,700 people and 275,000 balls per month, respectively). ${ }^{33}$ Profit rates vary across ball types but are generally low, approximately 8 percent at the median and 12 percent at the $90^{\text {th }}$ percentile. ${ }^{34}$ The corresponding firm size and profit margins in the full sample (Panel B) are slightly larger, indicating that the late responders are larger and more profitable than the initial responders. For most firms, all or nearly all of their production of size- 5 balls uses the standard "buckyball" design. The industry is relatively mature; firm age is 19.5 years at the median and 54 years at the $90^{\text {th }}$ percentile. Finally, cutters tend to have high tenure; the mean tenure in the current firm for a head cutter is 11 years (9 years at the median). One other salient fact is that the vast majority of firms pay pure piece rates to their cutters and printers. Among the initial responders, 79 of 85 firms pay a piece rate to their cutters, with the remainder paying a daily, weekly or monthly salary sometimes accompanied by performance bonuses. ${ }^{35}$

\section{Experiment 1: The Technology-Drop Experiment}

In this section we briefly describe our first experiment, the technology-drop experiment. For the purposes of the current paper, the first experiment mainly serves to provide evidence of low adoption, a puzzle we investigate using our second experiment, motivated in Section 5 and described in Section 6. As mentioned in the introduction, we originally planned to focus on spillovers of the technology to non-tech-drop firms. This in part explains why we treated a relatively small proportion of firms in the first experiment. We are planning to focus on spillovers in a companion project. ${ }^{36}$

The 85 firms in the initial-responder sample were divided into four strata based on quartiles of the number of balls produced in a normal month from the baseline survey. Within these strata firms were randomly assigned to one of three groups: the tech-drop group, the cash-drop group, and the no-drop group. We included the cash-drop group in order to shed light on the possible role of credit

\footnotetext{
${ }^{33}$ The employment numbers understate the true size of firms since the most labor intensive stage of production, stitching, is often done outside of firms in stitching centers or homes.

${ }^{34}$ For further discussion of the heterogeneity in profit rates (mark-ups) across firms, see Atkin, Chaudhry, Chaudry, Khandelwal, and Verhoogen (2015).

${ }^{35}$ In a later survey round, we also found that more than 90 percent of firms pay their printers a piece rate.

${ }^{36}$ Spillovers are thought to be a key mechanism generating increasing returns and they also provide the primary economic rationale for industrial policies to increase investment in innovation. To investigate such spillovers and their pathways, we collected detailed data on firm networks, as mentioned in Section 3. We chose the number of firms to be treated based on calculations for statistical power to pick up spillover effects. We are continuing to track information flows and adoption by non-tech-drop firms, and are planning to analyze the patterns in the companion project. But given the low adoption rates among the tech-drop firms — and hence the very limited adoption among firms who did not receive the technology — we decided to focus first on the puzzle of low take-up among the firms we gave the technology to. We chose not to do the second experiment on the non-tech-drop firms (cash-drop and no-drop groups) from the first experiment for the reasons discussed footnote 59. There are natural synergies between the two projects since, in the presence of misaligned incentives, we would predict that spillovers disproportionately originate from firms with better alignment of incentives.
} 
constraints in the technology-adoption decision. ${ }^{37}$ The top panel of Table 4 summarizes the distribution of firms across groups for the initial-responder sample. ${ }^{38}$ To increase sample size, we also randomized the initial non-responders into three groups using the same proportions as for the initial responders (treating them as a separate stratum). The bottom panel of Table 4 summarizes the response rates for the initial non-responders. It is important to note that response rates of the active initial non-responders are clearly correlated with treatment assignment: firms assigned to the techdrop and cash-drop groups were more likely to respond than firms assigned to the no-drop group. For this reason, when it is important that assignment to treatment in the tech-drop experiment be exogenous, we will focus on the initial-responder sample. In our second experiment, where we focus only on active tech-drop firms, all of which responded, this distinction will not be important.

We began the technology-drop experiment in May 2012. Firms assigned to the technology group were given a four-pentagon offset die, along with a blueprint that could be used to modify the die (Figure 4). They were also given a 30-minute demonstration, which involved first watching the firm's cutter cut a sheet using the traditional die and counting the number of panels, then instructing the cutter how to cut using the offset die and counting the panels. ${ }^{39}$ The die we provided cuts pentagons with edge-length of $44 \mathrm{~mm}$. As noted in Section 2, firms often use slightly different size dies, and the pentagon die size must match the hexagon die size. For this reason, we also offered firms a free trade-in: we offered to replace the die we gave them with an offset die of a different size, produced by a local diemaker of their choice. Firms could also trade in the four-panel offset die we gave them for a two-panel offset die (of the same or a different size). Of the 35 tech-drop firms, 19 took up the trade-in offer. All of these chose to trade in for the two-panel version. The cash group was given cash equal to the price we paid for each four-pentagon offset die, Rs 30,000 (US\$300), but no information about the offset die. Firms in the no-drop group were given nothing.

To examine baseline balance, Panel A of Table A.3 reports the mean of various firm characteristics across the tech-drop, cash-drop and no-drop groups for the initial-responder sample. We find no significant differences across groups. ${ }^{40}$ Panel B of Table A.3 reports the analog for the 31 late responders. Here we see significant differences for various variables, consistent with the observation above that response rates among this group are endogenous to treatment assignment.

Table 5 reports adoption rates as of August 2013, 15 months after we introduced the technology, with the initial-responder sample in Panel A and the full sample in Panel B. The first three rows

\footnotetext{
${ }^{37}$ In an experiment with micro-enterprises in Sri Lanka, de Mel, McKenzie, and Woodruff (2008) find very high returns - higher than going interest rates - to drops of cash of US\$100 or US\$200 (or of capital of roughly similar magnitudes), suggesting that the micro-enterprises operate under credit constraints. Although our prior was that the US $\$ 300$ value of the offset die would matter less to the larger firms in our sample, we included the cash-drop component in order to be able to separate the effect of the shock to capital from the effect of knowledge about the technology.

${ }^{38}$ At the moment of assignment, we believed that there were 88 active initial-responder firms with 22 in each stratum. In each stratum, 6 firms were assigned to the tech-drop group, 3 to cash-drop group and 13 to the no-drop group. Three firms that responded to our baseline survey subsequently either shut down or were revealed not to be firms by our definition, leaving 85 firms.

${ }^{39}$ We continued the demonstration until the cutter was able to cut 272 pentagons from a sheet. In almost all cases, this required one or two sheets; in no case did it require more than three.

${ }^{40}$ On average, firms in the technology group employ fewer people than other firms, but the differences are not statistically different at the 5 percent level.
} 
of each panel indicate the number of firms that were both active and responded to our surveys. The fourth row shows that a high proportion of tech-drop firms took up our offer of a trade-in for a different die, as mentioned above. The fifth and sixth rows report the number of firms that ordered and that received dies (beyond the one trade-in offered to tech-drop firms). The numbers are modest: in the full sample, one tech-drop firm and six no-drop firms made an additional order. (One diemaker was slow in delivering dies and firms canceled their orders, hence the discrepancy between the fifth and sixth rows.) The seventh and eighth rows report adoption as of August 2013 using the "conservative" and "liberal" adoption measures discussed in Section 3 above. Using the liberal adoption variable, in the full sample there were five adopters in the tech-drop group and one in the no-drop group. (In the initial-responder sample, the corresponding numbers are four and zero.) This number of adopters struck us as small. Given the apparently clear advantages of the technology discussed above, we were expecting much faster take-up among the firms in the tech-drop group.

We have investigated several alternative hypotheses for the low take-up, but found little evidence for the most common existing explanations. Lack of awareness of the technology (the assumption underlying "epidemic" models of diffusion, one of two main categories reviewed by Geroski (2000)) cannot be the explanation among tech-drop firms, since we ourselves manipulated the firms' information set. Another natural hypothesis is simply that the technology does not reduce variable costs as much as we have argued that it does. One piece of evidence against this hypothesis is the revealed preference of the six firms who adopted. Although these revealed preferences do not indicate the profitability of the technology among the full distribution of firms (as our calculations in Section $2.4 \mathrm{do}$ ), they are still instructive. In particular, the one adopter in the no-drop group, which we refer to as Firm Z, is one of the largest firms in Sialkot. This firm ordered 32 offset dies on 9 separate purchasing occasions between May 2012 and August 2013, and has ordered eight more dies since then. Figure A.10 plots the timing and quantity of its die orders. In March-April 2013 (Round 4 of our survey) the firm reported that it was using the offset die for approximately 50 percent of its production, and has since reported that the share has risen to 100 percent. It would be hard to rationalize this behavior if the offset die were not profitable for this firm.

We have estimated a number of simple linear probability models relating adoption as of August 2013 to measures of scale of production, quality of output produced, managerial ability and employee skill. Scale may be important to allow firms to spread fixed costs over more units. Quality may matter because the offset die generates greater cost saving for firms using more expensive, high-quality rexine. Manager and worker skill are often thought to be important for technology adoption. Tables A.4 and A.5 report the results. They can be briefly summarized: we do not find robust correlations between adoption and any of the covariates reflecting scale, quality, manager or employee skill. Given the small number of adopters as of August 2013, it is perhaps not surprising that we have not found robust correlations with firm characteristics. But we do interpret the results as deepening the mystery of why so few firms adopted the offset die. 


\section{Theory: Motivating Evidence and Summary}

In this section, we first discuss qualitative evidence that motivates our model of strategic communication in a principal-agent setting (Subsection 5.1). We then summarize the model and discuss its implications (Subsection 5.2). To save space, the formal exposition of the model appears in Appendix B (online).

\subsection{Motivating evidence}

Puzzled by the lack of adoption, in the March-April 2013 survey round we added a question asking tech-drop group firms to rank the reasons why they had not adopted the new technology, providing nine options (including an "other" category). Table 6 reports the responses for the 18 tech-drop firms that responded. Ten of the 18 firms reported that their primary reason for not adopting was that their "cutters are unwilling to work with the offset die." Four of the 18 said that their primary problem related to "problems adapting the printing process to match the offset patterns" and five more firms selected this as the second-most important barrier to adoption. This issue may be related to the technical problem of re-designing printing screens, but as noted above the cost of a new screen from an outside designer is approximately US $\$ 6$. It seems likely that the printing problems were related to resistance from the printers. (The other popular response to the question, to which most firms gave lower priority, was that the firm had received insufficient orders.)

The responses to the survey question were consistent with anecdotal reports from several firms. One notable piece of evidence is from the firm we have called Firm Z, the large adopter from the no-drop group, which is an exception to the local norm of piece-rate contracts. In part because of pressure from an international client, for several years the firm has instead paid a guaranteed monthly salary supplemented by a performance bonus, to guarantee that all workers earn at least the legal minimum wage in Pakistan. The fact that this large early adopter uses an uncommon incentive structure for cutters and printers seemed very suggestive.

We also feel that it is useful to quote at some length from field reports submitted by our own survey team. ${ }^{41}$ To be clear, the following reports are from factory visits during the second experiment, which is described in Section 6 below, and we are distorting the chronology of events by reporting them here. But they are useful to capture the flavor of the owner-cutter interactions that we seek to capture in the theoretical model. As described in more detail below, in our second experiment we offered one cutter in each firm (conditional on the approval of the owner) a lump-sum Rs 15,000 (US\$150) incentive payment to demonstrate competence in using the offset die. ${ }^{42}$ The following excerpts are all from firms in the group assigned to treatment for the second experiment.

In one firm, the owner told the survey team that he was willing to participate in the experiment but that the team should ask the cutter whether he wanted to participate. The report continues:

[The cutter] explained that the owner will not compensate him for the extra panels he will get out of each sheet. He said that the incentive offer of [Rs] 15,000 is not worth

\footnotetext{
${ }^{41}$ The team included our project manager, Tariq Raza, who wrote the reports, and staff of the RCONS survey firm.

${ }^{42}$ We also offered one printer per firm an incentive payment of Rs 12,000 (US\$120), as described below.
} 
all the tensions in future.

It appears in this case that the cutter, anticipating that the owner would not adjust his wage, sought to withhold information about the offset die in order to avoid a future decline in his pay. The cutter declined to participate and the firm was not treated.

In another firm, the owner, who had agreed to participate in the treatment, was skeptical when the enumerators returned to test the cutter. Our survey team writes,

[The owner] told us that the firm is getting only 2 to 4 extra pentagon panels by using our offset panel... The owner thinks that the cost savings are not large enough to adopt the offset die... He allowed us to time the cutter.

The team then continued to the cutting room without the owner.

On entering the cutting area, we saw the cutter practicing with our offset die... We tested the cutter... He got 279 pentagon pieces in 2 minutes 32 seconds... The cutter privately told us that he can get 10 to 12 pieces extra by using our offset die.

The owner then arrived in the cutting area.

We informed the owner about the cutter's performance. The owner asked the cutter how many more pieces he can get by using the offset die. The cutter replied, "only 2 to 4 extra panels."

It appears that the cutter had been misinforming the owner. But the cutter did not hide the performance of the die in the cutting process itself, likely either because it was difficult to do so or because he did not want to jeopardize his incentive payment.

The owner asked the cutter to cut a sheet in front of him. The cutter got 275 pieces in 2 minutes 25 seconds. The owner looked satisfied by the cutter's speed... The owner requested us to experiment with volleyball dies.

This firm subsequently adopted the offset die.

In a third firm, the owner reported that he had modified the wage he pays to his cutter to make up for the slower speed of the offset die. Our team writes,

[The owner] said that it takes 1 hour for his cutter to cut 25 sheets with the conventional die. With the offset die it takes his cutter 15 mins more to cut 25 sheets for which he pays him $[R s] 100$ extra for the day which is not a big deal.

This firm has generally not been cooperative in our survey, and we have not been able to verify that the firm has produced more than 1,000 balls with the offset die, and for this reason is not classified as an adopter. 


\subsection{Summary of model}

The survey results and qualitative evidence highlight the role of misaligned incentives and suggest that workers are discouraging some firms from adopting. But this raises two important questions. First, given that owners should be aware that workers have an incentive to discourage adoption of the offset dies, why are they influenced by what the workers say? Second, why do owners not simply offer a different labor contract, to give workers an incentive to support the adoption the cost-saving technology? To address these questions and to motivate our second experiment, we develop a model embedding a cheap-talk interaction along the lines of Crawford and Sobel (1982) in a standard principal-agent model. We recognize that other models with misaligned incentives and an ability of cutters to impose direct costs on firms may generate similar predictions. ${ }^{43}$ But in our view the model provides a particularly parsimonious account of the main forces at play. (As noted above, the full exposition is in Appendix B (online).)

We consider a principal (she) and an agent (he) in a one-time interaction. Production technologies are characterized by marginal cost, $c$, and speed, $s$. There is an existing technology, with $c_{0}$ and $s_{0}$. The principal is aware of the existence of a new technology, which may be of three types: $\theta_{1}$, which has the same costs as the existing technology but is slower; $\theta_{2}$, which is similar to our technology in that it has lower marginal cost than the existing technology but is slower (with the cost reduction more than offsetting the increased labor time); and $\theta_{3}$, which has the same marginal costs as the existing technology but is faster. The principal has priors $\rho_{1}, \rho_{2}$ and $\rho_{3}$ that the technology is of each type. ${ }^{44}$

In Stage 1, the principal must choose the contract she offers the agent. In Stage 2, Nature reveals the type of the new technology to the agent but not the principal. While this is clearly an extreme assumption, it captures in an analytically tractable way the observation from our qualitative work that the cutters are better informed about the cutting dies (which they work with all day every day) than are owners. In Stage 3, the agent can send a message to the principal about the technology. In Stage 4, the principal decides whether to adopt. In Stage 5, the agent chooses his level of effort. In Stage 6, output is observed, the technology is revealed to the principal, and payoffs are realized.

We consider two cases of the model, one in which the only available contract is a standard piece rate that must be chosen in Stage 1 (Subsection 5.2.1), and the other a "conditional" contract in which the piece rate can be conditioned on marginal cost, which is only revealed ex post, in Stage 6 (Subsection 5.2.2). We discuss the implications in Subsection 5.2.3 and the relation to our second experiment in Subsection 5.2.4.

\subsubsection{No conditional contracts}

In this case, we assume that the piece rate cannot be conditioned on information that is revealed later in the game, in particular on marginal cost. Given this, the agent strictly prefers faster tech-

\footnotetext{
${ }^{43}$ See in particular Dow and Perotti (2013) and Sections 4 and 5 of Garicano and Rayo (2016).

${ }^{44}$ We impose restrictions on the parameters of the model such that, for all the values of the piece rate that will be relevant, the principal, if she knew the technology type with certainty, would choose to adopt technology types $\theta_{2}$ and $\theta_{3}$ and not type $\theta_{1}$. In addition, the restrictions imply that the principal would not adopt the new technology given only her priors.
} 
nologies. Hence the principal and agent have a common interest to adopt type $\theta_{3}$ and not to adopt type $\theta_{1}$ but divergent interests over type $\theta_{2}$.

The analysis of the model is complicated by the fact that we have cheap talk embedded in a principal-agent interaction: for every choice of the piece rate there is a different cheap-talk subgame. But the analysis is greatly simplified by our first lemma (Lemma 1), which holds that in any subgame, modulo treating messages that induce the same action by the principal as equivalent (following Crawford and Sobel (1982)), there are just two possible subgame equilibria: a "babbling" equilibrium in which the principal ignores what the agent says and does not adopt; and an "informative" equilibrium in which the agent seeks to encourage adoption of type $\theta_{3}$ and discourage adoption of types $\theta_{1}$ and $\theta_{2}$, and the principal is influenced by the agent's message. We place no restrictions on the richness of the space of possible messages, but as shorthand we will refer to a message that encourages the principal to adopt as "technology is good" and one that discourages her as "technology is bad." In the informative subgame equilibrium (if it exists), if the principal hears the message "technology is bad", she infers that the technology is type $\theta_{1}$ or $\theta_{2}$; if "technology is good", she infers $\theta_{3}$. One implication of Lemma 1 is that there cannot exist an equilibrium in which the agent fully reveals the technology type to the principal.

Our key proposition in this case (Proposition 1) holds that there exists an equilibrium in which the principal offers the same piece rate as under the existing technology, the agent says "technology is bad" if it is type $\theta_{1}$ or $\theta_{2}$ and "technology is good" if it is type $\theta_{3}$, and the principal follows the agent's advice. In particular, if the technology is type $\theta_{2}$ the agent discourages the principal from adopting and she does not to adopt. ${ }^{45}$ It is common in the literature on cheap talk to assume that players will coordinate on the most informative equilibrium in cheap-talk interactions. ${ }^{46}$ If we adopt this assumption (for every cheap-talk subgame), then the equilibrium described in Proposition 1 is unique.

\subsubsection{Conditional contracts}

In this case, we allow the principal at Stage 0 to pay a fixed cost, $G$, to be able to offer a contract that conditions the piece rate on marginal cost, revealed in Stage 6. Given our assumptions, only type $\theta_{2}$ differs in marginal cost from the existing technology. The conditional contract is useful in that it enables the principal to offer a higher piece rate if the technology is type $\theta_{2}$. The fixed cost $G$ can be interpreted as the cost of a commitment device to pay a piece rate above the one that would be paid for the existing technology $\left(\beta_{0}\right)$, which otherwise the principal would not be able to credibly commit to. (We discuss other possible interpretations in Section 5.2.3 below.)

\footnotetext{
${ }^{45} \mathrm{~A}$ word of explanation about whether the equilibrium involves "misinformation": formally, the actual words that the agent uses to convey his message are immaterial; what matters is how the principal interprets the message, and in the Proposition- 1 equilibrium the principal interprets "technology is bad" to mean "technology is type $\theta_{1}$ or $\theta_{2} . "$ In this sense, the agent technically does not "misinform" the principal about type $\theta_{2}$ and we avoid using this terminology in the theory. But in the empirical setting we have found that workers tend to use particular words to discourage adoption that paint the type $\theta_{2}$ technology as ineffective, and describing the communication as misinformation there is more apt.

${ }^{46}$ Crawford and Sobel (1982) argue that the most informative equilibrium is "a reasonable one for agents to coordinate on" because it is Pareto-optimal in their setting. Other justifications for the assumption have also been discussed in the literature; see e.g. Chen, Kartik, and Sobel (2008).
} 
Our main proposition in this case (Proposition 2) holds that there exists an equilibrium in which, if the contracting fixed cost $G$ is sufficiently small, the principal pays $G$ and offers a supplement to the piece rate if the technology is revealed to by type $\theta_{2}$. Given the supplement, the agent wants to adopt the same technologies that the principal wants to adopt, $\theta_{2}$ or $\theta_{3}$, and technologies of type $\theta_{2}$ are adopted.

"Sufficiently small" for $G$ means less than the expected benefit of adopting technology $\theta_{2}$ relative to the existing technology, which depends on speed and cost of the technologies as well as the principal's prior, $\rho_{2}$, that the technology is type $\theta_{2}$. If $\rho_{2}$ is sufficiently low, then the principal will not pay $G$ and the players will be back in the world of the first case, where their interests diverge and type $\theta_{2}$ will not be adopted. Again, if we assume players coordinate on the most informative subgame equilibria, then the Proposition-2 equilibrium is unique.

\subsubsection{Discussion}

Let us come back to the two questions posed at the beginning of the model summary. First, why are owners influenced by cutters' messages, given that they should be aware that cutters have an incentive to resist adoption of our die? The answer from the model is that the interests of the principal and agent are sufficiently aligned — they have common interests over types $\theta_{1}$ and $\theta_{3}$ - that the agent's message can be informative. In particular, in the Proposition-1 equilibrium, the principal can infer whether $\theta=\theta_{3}$ or $\theta \in\left\{\theta_{1}, \theta_{2}\right\}$, and this is valuable enough that she pays attention to the agent's message, knowing that it may lead her not to adopt type $\theta_{2}$.

Second, why don't owners offer a labor contract that incentivizes cutters to support the adoption of our die? The model suggests two reasons. One is simply that the principal may be unaware of the existence of the conditional contract; this corresponds to the no-conditional-contracts case. The conditional contract may be an organizational innovation that was previously unknown, at least to some firms, in the same way that our technical innovation was previously unknown.

Another reason, corresponding to the conditional-contracts case, is that the principal is aware of the conditional contract but perceives the cost of implementing it to be higher than the expected benefit. The contracting fixed cost, $G$, can be interpreted in a number of ways. It may be that a fairness norm has arisen around standard piece-rate contracts, such that any deviation would carry a morale cost. Or firms may worry that if they offer a higher piece rate in one case, workers will demand higher rates in others. $G$ can also be interpreted as a cost of a commitment device for the principal's pledge to pay the higher piece rate if the technology is type $\theta_{2} \cdot{ }^{47}$ Finally, the fixed cost can be interpreted in light of the ratchet effect (e.g. Gibbons (1987)). If a worker on a piece-rate contract discovers a labor-saving innovation, he may not reveal it to the owner if he expects her to cut the piece rate in response. It may be optimal for the principal to commit to not changing the piece rate in order to encourage labor-saving innovations. If most innovations in Sialkot are labor-saving, this may explain why piece rates are sticky and why it may be costly for firms to start offering conditional

\footnotetext{
${ }^{47}$ In our model, marginal cost is revealed in Stage 6, after effort has been supplied in Stage 5. Since the game is one-shot, the principal would have an incentive not to pay the higher piece rate as promised. (This incentive would be present under the existing technology as well, but it seems plausible that it would be more difficult for firms to avoid paying the going rate in the industry, which here corresponds to the optimal piece rate under the existing technology.)
} 
contracts - contracts that open the door to ratcheting. In Section 7 below, we discuss the previous cutting innovation in Sialkot, which was labor-saving. It seems plausible for firms to expect other new technologies to be labor-saving as well, and to be reluctant to modify piece rates for this reason.

These two possible explanations for the stickiness of labor contracts - that owners are not aware of the existence of conditional contracts, and that the benefits owners expect do not outweigh the costs of adopting them - have similar implications for the players' behavior. In either case, the principal offers a pure (non-conditional) piece rate contract and a technology of type $\theta_{2}$ is not adopted. The key point is that, for whatever reason, many owners did not in fact adjust labor contracts. This left scope for our incentive intervention, described below, to have an effect.

This discussion is related to a broader debate in organizational economics about the underlying causes of organizational failure, of which the lack of adoption of our dies is arguably an example. An organization may fail because it was poorly designed ex ante. Or its design may represent an ex-ante optimal solution to trade-offs between uncertain costs and benefits, but a shock yields a sub-optimal outcome ex post. In our context, the idea that an owner is simply not aware of the existence of conditional contracts corresponds to the first view. The idea that the owner perceives the fixed cost of offering a conditional contract to be greater than the expected benefit of adopting a type- $\theta_{2}$ technology corresponds to the second. Our preferred interpretation is the latter; we believe that most owners are aware of the possibility of conditional contracts, but choose not to offer them because they assign low probability to the arrival of beneficial new technologies like our dies. But we recognize that the two interpretations predict similar behavior and it is difficult to discriminate between them empirically. ${ }^{48}$

\subsubsection{Motivation for incentive intervention}

Testing the implications of our model presents a number of practical challenges. In principle, one approach would be to pay the fixed cost, $G$, for firms and examine whether they change the labor contract and adopt the technology. But this cost is not observable and depends on complex social dynamics within firms; it is not clear how much we (the experimenters) would pay, or to whom. Another approach would be to offer a piece-rate supplement ourselves. But firms are reluctant to share the detailed production information that would be required to implement such a payment. The challenges we faced simply in surveying firms indicated to us that it would be impossible to manipulate the piece rate directly in our second experiment. ${ }^{49}$

Facing these constraints, we opted for a third approach: we offered a one-time lump-sum payment to one cutter and one printer per firm, conditional on revealing the offset die to be cost-saving in front of the owner. (Presumably, other non-monetary inducements such as increased authority or greater work schedule flexibility could have served a role similar to our one-time lump-sum

\footnotetext{
${ }^{48} \mathrm{~A}$ third view should be considered: that the pure (non-conditional) piece rate is a first-best contract (with no misalignment of incentives) ex ante, and the misalignment arises only because of the intervening shock. In our model, this can be interpreted as a limiting case of the second view above, where ex ante the owner is certain that the technology is not of type $\theta_{2}$ (that is, $\rho_{2}=0$ ).

${ }^{49}$ In the end, one-third of firms chose not to participate in the much less invasive intervention we decided to implement; see Section 6 below for details. This confirmed our earlier belief that firms' willingness to participate would be limited.
} 
payment.) In Appendix B.4, we prove a formal proposition that if such a conditional lump-sum payment by a third-party experimenter is sufficiently large, then there exists an equilibrium in which the agent encourages adoption of type $\theta_{2}$ and the principal adopts it, even if she would not have offered the conditional contract on her own. Intuitively, the incentive payment plays the same role as the supplement to the piece rate in the conditional-contracts case: it raises the payoff to the agent of revealing the technology to be type $\theta_{2}$. Empirically, the main implication is that we would expect a sufficiently large lump-sum incentive payment to increase adoption of the offset die. It is worth noting that "sufficiently large" in this context is relative to the possible wage losses of a single cutter, not to the revenues or profits of a firm. Indeed, we will see below that a lump-sum incentive payment that appears small from the point of view of firms has a large effect on adoption. ${ }^{50}$

\section{Experiment 2: The Incentive-Payment Experiment}

\subsection{Experimental design}

Motivated by the theoretical model, we conducted our second experiment, the incentive-payment experiment, in September-November 2013. To avoid interfering with the process of diffusion to the non-tech-drop firms that is the focus of our companion project on spillovers (see footnote 36), we focused on the 35 tech-drop firms (including both initial responders and initial non-responders). The 31 still-active firms among these were divided into four similarly sized strata: ${ }^{51}$ (1) firms in the two smaller strata from the tech-drop experiment that had not yet adopted the die as of August 2013; (2) firms in the two larger strata from the tech-drop experiment that had not yet adopted the die; (3) firms from the initial non-responder stratum from the tech-drop experiment that had not yet adopted the die; and (4) firms that had already adopted the die. ${ }^{52}$ Within each stratum, firms were randomly assigned with equal proportion to a treatment subgroup (which we call Group A) and a control subgroup (Group B). There were 15 firms assigned to Group A and 16 to Group B.

To firms in Group B we gave a reminder about the offset die and the new cutting pattern, and explicitly informed them about the two-pentagon variant of the offset die (which, as noted above, had proven more popular than the four-pentagon offset die we originally distributed.) We also offered to do a new demonstration with their cutters. To each firm in Group A, we gave the same refresher, the same information about the two-pentagon variant, and the same offer of a new demonstration. In addition, we explained the misalignment of incentives to the owner and offered to pay one cutter and one printer lump-sum bonuses roughly equivalent to their monthly incomes — 15,000 Rs (US\$150) and 12,000 Rs (US\$120), respectively - on the condition that within one

\footnotetext{
${ }^{50}$ Note, however, that the required size of the lump-sum transfer may vary across workers, depending on individual and firm characteristics. In the first anecdote above, for instance, it appears that the proposed payment was not large enough to induce the cutter to report accurately. In all other cases it appears that the proposed transfer was large enough to induce cutters to participate in the intervention.

${ }^{51}$ At the time of randomization, we believed that 34 of these firms were still active. Three of these were subsequently revealed to have stopped manufacturing soccer balls.

${ }^{52}$ As shown in Table 5, there were four adopters as of August 2013 in the full sample using the conservative definition (which is based on our survey data), and five adopters according to the liberal definition (which combines survey data with field reports submitted by our enumerators). We stratified using the liberal definition, our preferred measure.
} 
month they demonstrate competence in using the new technology in front of the owner. If the owner agreed to the intervention, we explained the intervention to one cutter and one printer chosen by the owner, paid them $1 / 3$ of the incentive payment on the spot, and scheduled a time to return to test their performance using the die. ${ }^{53}$

The performance target for cutters was 272 pentagons from a single sheet in three minutes using the offset die. The target for the printer was 48 pairs of offset pentagons in three minutes. ${ }^{54}$ We provided the owner with 20 laminated rexine sheets and printing screens for offset pentagon pairs for his workers to practice with, and a nominal Rs 5,000 (US\$50) to cover additional costs such as overhead (e.g. electricity while the cutters were practicing). We returned after approximately one month to test the employees and, upon successful achievement of the performance targets, to pay the remaining $2 / 3$ of the incentive payments. Without revealing ahead of time that we would do so, we allowed for a buffer of 30 seconds and 5 pentagons for cutters and 30 seconds for printers. ${ }^{55}$

Table 7 evaluates baseline balance by comparing firm characteristics across Group A and Group B firms at the time of our visit to explain the intervention (September 2013). No differences in means are statistically significant. It appears that the randomization was successful. ${ }^{56}$

\subsection{Results}

Ten of the 15 Group A firms agreed to participate in the experiment. ${ }^{57}$ Table A.6 reports the times achieved by the chosen cutter at each firm (all using the two-pentagon variant of the offset die). The average time was 2 minutes and 52 seconds, approximately 27 percent longer than the typical time to cut with the traditional die ( 2 minutes and 15 seconds). For this reason, we believe that the 100 percent increase in labor time factored into the cost calculations in Section 2 is conservative. ${ }^{58}$ In addition, many cutters expressed confidence that with additional use they could lower their cutting time. All printers easily achieved their target, consistent with the assumption in Section 2 that, despite some printers' fears, the offset die does not increase labor time for printing.

To measure short-run adoption responses, we carried out a survey round in January-March 2014 (Round 6 of our survey), 2-5 months after the completion of the incentive-payment intervention. Before turning to the formal regressions, it is useful to examine the raw adoption statistics. Of the 10 Group A firms that agreed to participate in the experiment, two had already adopted the die.

\footnotetext{
${ }^{53}$ To the extent possible, we attempted to make the payment directly to the cutter and printer. In two cases, the owner insisted that we pay him and that he pass on the money to the employees, and we acceded to this request.

${ }^{54}$ The 3-minute targets were chosen after conducting speed tests at two of the pilot firms mentioned in Section 4. They are approximately 33 percent higher than the time to cut a single sheet using the original die and the time to print 48 two-pentagon panels cut using the original die.

${ }^{55}$ That is, the effective target for cutters was 267 pentagons from one sheet in 3 minutes 30 seconds, and for printers was 48 pairs in 3 minutes 30 seconds.

${ }^{56}$ Because of an error by our enumerators, one firm that was assigned to Group B was offered the incentivepayment intervention. This occurred while two co-authors of the paper were in the field, and the error was caught within hours of its occurrence. To maintain balance, we randomly selected one as-yet-untreated Group A firm from the same stratum and re-assigned it to Group B. As neither firm adopted, our results hold with the original randomization (available upon request).

${ }^{57}$ In two of these 10 firms, it was not possible to complete the printer performance test.

${ }^{58}$ The cutters were under high-powered incentives to cut a sheet in under 3 minutes but, in contrast to their regular piece-rate incentives, had no incentive to cut faster than that. Given this ambiguity, we chose a very conservative 100 percent increase.
} 
Of the remaining eight firms, five subsequently adopted using the liberal definition (or four using the conservative definition). Of the 16 Group B firms, three firms had already adopted prior to the invention. None of the remaining 13 firms adopted in the short run, by either measure. To examine medium-run responses, we carried out another survey round in October-December 2014 (Round 7 of our survey), 11-13 months after the completion of the intervention. If the technology is beneficial, as we have argued, one would expect it eventually to be adopted by all firms (once there has been sufficient social learning, for instance) and there would then be no long-run effect of our incentive treatment. In this sense, it would be reasonable to expect the medium- and long-run treatment effect to be smaller than the short-run one. Indeed, by Oct.-Dec. 2014, one initial non-adopter in Group B (that is, a Group B firm that had not yet adopted at the start of Experiment 2) had adopted the die (by either definition).

Table 8 uses the liberal adoption measure to assess formally the impact of the incentive-payment intervention on adoption rates. All regressions include dummies for the four strata described above. Panel A reports impacts on adoption in the short run, and Panel B in the medium run. Focusing on the short run first, the first-stage estimates (Column 1) indicate, not surprisingly, that assignment to Group A is significantly associated with greater probability of receiving the incentive-payment treatment; that is, we have a strong first stage. The dependent variable in Columns 2-4 is a 0/1 indicator for whether a firm has adopted. The OLS estimate of the coefficient on treatment in Column 2 is positive and significant, but one might be worried about selection into treatment. The reduced-form (intent-to-treat, ITT) result in Column 3 does not suffer from such selection issues and indicates a positive and significant (at the 5 percent level) causal relationship between assignment and adoption. The estimate indicates that the probability of adoption increased by 0.32 among those assigned to treatment (from a baseline of 13 percent). ${ }^{59}$ The IV estimate (the effect of treatment on the treated) is substantially higher: 0.48. However, since the one third of firms who refused the intervention may have chosen to do so because of particularly large costs of adoption (or small benefits), these IV estimates should be treated with caution. The medium-run ITT estimate in Panel B is slightly lower at 0.27 (and statistically significant at the 10 percent level) because of the one Group B firm that adopted in the second six-month period after the intervention. ${ }^{60}$ Table 9 reports very similar results using the conservative adoption measure - the short-run ITT is 0.31 and statistically significant at the 5 percent level, and the medium-run ITT is 0.26 and statistically significant at the 10 percent level. ${ }^{61}$

\footnotetext{
${ }^{59}$ An interesting question to consider is how our estimates would differ if we had included the non-tech-drop firms in our second experiment - that is, if we had offered the technology and incentive payment at the same time to firms that had not received the offset die initially. We suspect that the ITT estimate would have been larger among these firms. Among the original tech-drop firms, cutters that did not reveal that the technology was a good one before Experiment 2 may have been particularly reluctant to do so in Experiment 2, since it would have revealed that they had previously been mis-reporting the benefits of the technology. By contrast, if we had given the technology and incentive simultaneously to the original non-tech-drop firms, cutters in those firms would have had no reason to be reluctant. Because of this difference, treating the non-tech-drop firms in Experiment 2 would not have represented a simple increase in sample size; we believe that it would not have been appropriate to pool the data from the tech-drop and non-tech-drop groups.

${ }^{60}$ The number of observations falls by two in the medium-run results because two firms exited.

${ }^{61}$ Tables A.7-A.9 re-run the results using more stringent adoption thresholds of 5,000, 10,000 or 20,000 balls,
} 
To check robustness, Table 10 reports results using an alternative indicator of adoption: whether the firm purchased its first offset die (beyond the trade-in that we paid for) after September 1, 2013. Of the eight firms that accepted the intervention and had not adopted by August 2013, three subsequently purchased their first offset die. (One of these firms had not yet produced with it at the time of our Oct.-Dec. 2014 survey round.) ${ }^{62}$ Table 10 shows that the positive causal effect of the incentive-payment treatment on adoption is robust to using this alternative measure. As before, we report both short- and medium-run impacts. The short- and medium-run ITT estimates are 0.27 and 0.28 , respectively, and are both statistically significant at the 5 percent level.

It is important to acknowledge that the sample sizes in the incentive-payment experiment are small. An alternative to large- $N$ statistical inference are permutation tests whose properties are independent of sample size. (See Bloom et al (2013) for the use of this type of inference in a similar context.) We determine the proportion of all 25,872,000 possible treatment assignments that would produce coefficients as large as or larger than the ones we find (holding the observed outcome for each firm unchanged). ${ }^{63}$ This procedure does not require asymptotic approximations. Given the selection discussion above, we focus on the ITT estimates in Column 3 of Tables 8-10. Figure A.11 plots the distribution of coefficients obtained from regressing the liberal adoption measure on assignment to Group A for the millions of possible treatment assignments. Because of the small number of adopters, there are only a handful of possible coefficients, despite the large number of possible assignments. For the short run, our estimated ITT is the most extreme coefficient that could be observed under any treatment assignment and is equal to the critical value for a 1 percent significance test. For the medium run, where we would expect the treatment effect to have begun to attenuate, a fraction 0.061 of assignments are more extreme and our point estimate is equal to the critical value for a 10 percent significance test. Figure A.12 reports the corresponding plots using the conservative adoption measure. Our short-run ITT estimate lies between the 1 and 5 percent critical values, with a fraction 0.018 of assignments being more extreme. Our medium-run ITT estimate lies just below the 10 percent critical value with a fraction 0.118 of assignments being more extreme. Figure A.13 presents a similar analysis for our alternative indicator of adoption, die purchases. The short-run ITT estimate is the most extreme outcome possible and is equal to the 1 percent critical value. The medium-run ITT estimate is equal to the 5 percent critical value, with a fraction 0.036 of assignments being more extreme.

To sum up: although the level of significance dips slightly below 90 percent in one of the specifications (using the more conservative adoption measure and the small-sample-robust permutation

respectively (as opposed to the 1,000 ball threshold used in Tables 8-9). The results are even stronger because no control firm is classified as an adopter using these cutoffs. Note that the adoption rates are the same for the 5000 and 10000 cutoffs, so the numbers in those tables are identical to each other.

${ }^{62}$ In addition, one large Group-A firm that was already classified as an adopter because it was using the offset cutting pattern for table cutting (see footnote 19), purchased its first die (beyond the four-panel offset die we originally gave) following the beginning of our intervention.

${ }^{63}$ Within each of the four strata, we assigned treatment status with equal proportion. The stratum of smaller firms contained 6 firms, the stratum of larger firms contained 12 firms, the stratum of initial non-responders contained 8 firms and the stratum of already-adopters contained 5 firms. This means there are $25,872,000=\left(\begin{array}{l}6 \\ 3\end{array}\right)\left(\begin{array}{c}12 \\ 6\end{array}\right)\left(\begin{array}{l}8 \\ 4\end{array}\right)\left(\left(\begin{array}{c}5 \\ 2\end{array}\right)+\left(\begin{array}{l}5 \\ 3\end{array}\right)\right)$ possible treatment assignments. 
test in the medium-run specification, when we would expect the effect to have begun to attenuate), overall the results strongly suggest that, consistent with our theoretical model of misaligned incentives, the incentive-payment treatment spurred adoption among firms.

\section{Additional Evidence on Mechanisms}

In this section, we present additional (non-experimental) evidence on two key features of our theoretical model: wage stickiness and information flows within the firm. So as not to reveal the existence of the offset die to non-tech-drop firms, most of the questions we discuss were asked only of the 31 tech-drop firms involved in the second experiment, and we focus on these tech-drop firms throughout this section. ${ }^{64}$

To shed light on wage stickiness, we recorded the wages of cutters and printers for each month between August 2013 and September 2014. Generally, it appears that firms change wages relatively seldom. Of the 24 tech-drop firms that provided complete information, 10 did not change the head cutter's wage at all over the entire 13 month period, a period of 8.4 percent annual inflation (Table A.10). Thirteen out of 24 did not change the wage for the head printer. This evidence is corroborated by the survey responses from the head cutters and printers themselves: only 2 out of 15 cutters who responded indicated that their wage had changed during this period, and only 4 out of 17 printers reported a change. We asked firms why they changed wages, and the responses for the few firms that provided answers are in Table A.11. The two main reasons cited by owners were (a) inflation and (b) the change was a regular "end of year" change. Only one firm reported changing wages because of the offset die. In addition to the reasons discussed in Section 5.2.3 above, another possible reason for the wage stickiness is that it is conventional to use round numbers for piece rates. Figure A.14 shows that 78 percent of piece rates are 1, 1.5, 2, 2.5, 3 or 4 Rupees per ball. Given this convention, it may be that wage increases must be reasonably large when they occur. These several pieces of evidence reinforce the idea that piece rates are sticky, consistent with the ideas (a) that they are chosen in Stage 1 of the game, prior to the technology arriving, and (b) that there is a significant cost to adopting contracts that involve changing piece rates.

We also asked owners and head cutters directly why payments were not conditioned on the technology and whether such a possibility was ever discussed. The reason cited most often by owners (6 out of 18) is that offering such incentives would lead workers to expect additional incentives in the future. The next two most common answers were that workers would perceive these incentives as unfair or that the owners did not think of it (Table A.12). Cutters overwhelmingly state that they feel it is not their place to make suggestions to the owner about the payment schemes (Table A.13). In almost no case did the owner report discussing payments to adopt the die with any employee (Table A.14). Similarly, cutters never report talking to the owner or other co-workers about payments to adopt the die. These responses are consistent with both of the hypotheses for lack of adjustment of contracts discussed above: that owners and cutters were largely unaware of the possibility of conditional contracts, or that both were aware of the contracts but that there

\footnotetext{
${ }^{64}$ This section examines responses from Round 7 of our survey, conducted in Oct.-Dec. 2014.
} 
were transaction costs associated with adopting them.

Turning to information flows within the firm, we asked owners if they had ever had a conversation with their head cutter, other cutters or head printer about whether to adopt the offset die. ${ }^{65}$ It appears that this question did not capture all forms of communication between owners and cutters. For instance, of the 10 firms that reported "cutters unwilling" as the main reason for not adopting the technology in Round 4 of our survey (Table 6), only six reported that they had had a conversation with the head cutter about whether to adopt the die. ${ }^{66}$ Presumably the remaining respondents received signals from cutters through less direct means, or were too proud to admit to taking advice from subordinates. With this caveat, the responses do suggest that owners are being swayed by the opinions of cutters in their adoption decisions. Ten of 22 respondents reported consulting with their head cutter about the decision (Table A.15). There is a clear association between what the cutter recommends and what owners eventually do. Table A.16 reports the recommendations given by the cutters in the 10 cases where owners report consulting them, and the firms' corresponding adoption decisions using the liberal definition. In all three instances where the head cutter recommended adopting, the firm adopted; in four of the six instances where the head cutter's recommendation was negative, the firm did not adopt. ${ }^{67}$ These responses are consistent with the assumption in the model that owners are less informed than cutters, and the result that in some circumstances the principal finds it worthwhile to follow the cutter's advice.

As mentioned above, additional corroborative evidence for our story comes from firms' experience with a previous innovation in the cutting process. Prior to 1994, all firms used a single-panel die to cut pentagons. In 1994, the first firms began using the two-panel non-offset pentagon die pictured on the right-hand side of Figure 1, referred to in Sialkot as the "back-to-back" die. This die is much faster than a single-pentagon die, since each strike by the hydraulic press cuts two pentagons, and the die does not appear to have reduced the number of pentagons per sheet. Owners report that the back-to-back die was adopted much more quickly than our offset die has been, and with little resistance from cutters. All 14 owners who could recall the speed of adoption indicated that they adopted the die within six months of hearing about it (Table A.17). Furthermore, 23 out of the 24 respondents report there was no resistance among cutters to adopt this die (Table A.18) and very few firms changed their payment structure in any way (Table A.19). Relating this evidence to our theory, the back-to-back die can be interpreted as corresponding to technology type $\theta_{3}$ (equal marginal cost but faster than the existing technology), which both principal and agent want to adopt. The fact that the back-to-back die was adopted quickly and with little resistance from cutters reinforces two ideas from the theory: first, that cutters' and owners' interests over some new technologies coincide (a precondition for informative communication); and second, that the misalignment of incentives for our offset die is (at least in part) responsible for the slow adoption of it.

\footnotetext{
${ }^{65}$ The exact wording of the questions were "Did you have a conversation with [the following employee] about whether you should adopt the offset die?" and "If yes, what did they say?" with response codes "(a) the technology is beneficial and should be adopted, (b) the technology is not beneficial and should not be adopted, (c) not sure whether the technology is beneficial or not, $(\mathrm{d})$ other (----)."

${ }^{66}$ In all six cases, the cutters recommended against adoption.

${ }^{67} \mathrm{Using}$ the conservative definition, in five of the six cases the firm did not adopt.
} 


\section{Alternative Hypotheses}

In this section, we examine what we consider to be the two leading competing explanations for the results of our second experiment: (1) that we mechanically induced firms to adopt by subsidizing the fixed costs of adoption, rather than by aligning incentives and inducing information flows within firms; and (2) that we simply increased the salience of the new technology and this in itself led firms to adopt.

\subsection{Subsidies for fixed costs}

Suppose in our model that the principal and agent had the same information about the technology. The owner would then simply weigh the expected variable cost reduction from the new technology against the fixed costs of adoption, $F$. These fixed costs might include a lump-sum bonus to workers to compensate them for a learning period in which their incomes are reduced. Our incentive-payment experiment could be seen as providing a subsidy for these fixed costs, and the subsidy may itself have induced the owner to adopt.

Is this a quantitatively plausible explanation of our findings? To organize our thinking about this question, we can write the present discounted value of expected additional profit from adoption for firm $f$ as follows:

$$
\Pi_{f}=-F_{f}+P \sum_{t=1}^{\infty} \frac{N V B_{f}}{(1+r)^{t}}=-F_{f}+P\left(\frac{N V B_{f}}{r}\right)
$$

where $\Pi_{f}$ is expected profit, $F_{f}$ are fixed costs of adoption, which must be paid up front and may be firm-specific; $P$ is the probability perceived by the firm that the technology works as we said it does; $N V B_{f}$ are net variable benefits per cutter per month; and $r$ is the market interest rate. ${ }^{68}$ Net Variable Benefits can be calculated for each firm following the method of Section 2.4; we assume that these are constant over time. For firms that did not have to buy the die (i.e. the tech-drop firms) a conservative estimate of "observed" fixed costs is Rs $10,800 / \mathrm{US} \$ 108 .{ }^{69}$ In this section, we make the assumption that the Rs $32,000 / \mathrm{US} \$ 320$ we paid in the incentive-payment treatment were also necessary and part of the true fixed cost of adoption. ${ }^{70}$ We thus take the total observed fixed costs to be Rs $42,800 / \mathrm{US} \$ 428 .{ }^{71}$ Including the incentive payments in fixed costs seems conserva-

\footnotetext{
${ }^{68}$ Here we are abstracting from the possibility that firms can learn from others about the profitability of adoption, which would tend to lead them to delay adoption (and wait for others to adopt), as emphasized by Foster and Rosenzweig (1995). Such an effect could help to explain low initial adoption, but not the large increase in adoption in response to our second experiment, and the argument of this section that our subsidies were too small to generate the latter would continue to hold in a more complex social-learning model.

${ }^{69}$ As discussed in Section 2.4, the fixed costs are Rs 800/US\$8 to have screens redesigned and remade and Rs $10,000 / \mathrm{US} \$ 100$ for an offset die for the combing machine.

${ }^{70}$ As discussed in Section 6.1 , we paid Rs $15,000 / \mathrm{US} \$ 150$ to the cutter, Rs $12,000 / \mathrm{US} \$ 120$ to the printer, plus Rs $5,000 /$ US $\$ 50$ to the owner to cover overhead. In Section 2.4, we assumed that workers wages went up by 100 percent to compensate them for using the slower technology. The discussions in Sections 5 and 7 suggest that many firms do not adjust their wages and so in this section we assume that firms make no changes in piece rates but make a one-off lump-sum payment to cutters and printers of the same size as in our Experiment 2.

${ }^{71}$ We take these to be the fixed costs per cutter. This is a conservative assumption since it is possible that firms could, for example, share the combing machine across cutters.
} 
tive; in the incentive-payment experiment, only one worker felt that the payment was insufficient to cover his costs of adoption, suggesting that the incentive payments were greater than the "training" cost faced by workers in the great majority of firms.

As a first step toward answering the quantitative plausibility question, suppose that there is no uncertainty about the technology (i.e. $P=1$ ) and no unobserved fixed costs (i.e. no fixed costs beyond the US $\$ 428$ mentioned above.) Under these assumptions, equation (1) can be reconciled with non-adoption only if firms face extremely high interest rates and hence have a small effective discount factor $1 /(1+r) .{ }^{72}$ The values of firm-specific interest rates that set $\Pi_{f}=0$ in (1) represent lower bounds on the firm-specific interest rates for non-adopters. At the $10^{\text {th }}$ percentile, the lower bound is 13.8 percent per month; at the $90^{\text {th }}$ percentile, the lower bound is 134 percent per month. We asked firms explicitly about the interest rates they face, and the responses ranged from 9 percent to 25 percent per year; these are an order of magnitude lower than the implied lower bound for most firms. That is, in the absence of both uncertainty about the technology and unobserved fixed costs, the interest rates (or rate of discounting) that would be required to explain the low initial rates of adoption appear to be implausibly high.

This argument leaves open the possibility that some combination of uncertainty and unobserved fixed costs can account for the low initial rates of adoption we observed. To address this possibility, we take a different approach: we allow for both uncertainty and unobserved fixed costs and ask whether these can explain both the low rates of initial adoption and the magnitude of the response to our incentive-payment intervention. The unobserved fixed costs may represent attention costs for the owner or psychic costs involved in changing established routines. We assume, conservatively, that the interest rate is the highest of the self-reported interest rates, 25 percent per year. As a benchmark, we assume that owners place a 50 percent probability on the event that the technology works as we described (i.e. $P=0.5$ ); we consider alternative priors below. Under these assumptions, we can place bounds on the values of unobserved fixed costs that can explain the behavior we observe. In particular, from (1), if a firm does not adopt initially, it must be that $\Pi_{f}<0$ and hence that $F_{f}>(0.5) \frac{N V B_{f}}{0.0187}$. (A 25 percent annualized interest rate corresponds to a 1.87 percent monthly rate.) If a firm adopts in response to the incentive-payment experiment, it must be that $\Pi_{f}>0$ after the US\$320 reduction in fixed costs, and hence that $F_{f}<(0.5) \frac{N V B_{f}}{0.0187}+320$. These bounds on fixed costs are plotted by rank in Figure 5 for the 31 firms in the incentive-payment experiment (using the liberal adoption measure). The solid black bars indicate upper bounds on fixed costs for the initial adopters (for which we can only calculate upper bounds). The white bars indicate lower bounds for the never-adopters (for which we can only calculate lower bounds). The solid grey bars indicate lower bounds for initial non-adopters that adopted in response to the incentive treatment (i.e compliers in Experiment 2); the black outlines above the solid grey bars indicate upper bounds for these firms. The figure highlights that the US $\$ 320$ subsidy (indicated by the distance between the top of the solid grey bars and the black outlines just above them) is small relative to the implied lower bound on fixed costs for almost all initial non-adopters.

\footnotetext{
${ }^{72}$ This is another way of stating the argument from Section 2.4 that the observable fixed costs of adoption can be recouped within a relatively short amount of time by almost all firms.
} 
In this context, the key question is whether a plausible distribution of unobserved fixed costs is consistent with the adoption responses and implied bounds illustrated in the figure. It turns out that if we impose even minimal structure on the distribution of unobserved fixed costs then it is very unlikely that the distribution would generate both the initial lack of adoption (which requires that unobserved fixed costs be large) and the large response to the second experiment (which requires that US $\$ 320$ make a significant difference to the adoption decisions of a large number of firms). Suppose that fixed costs are distributed log normally:

$$
\ln \left(F_{f}\right)=\mu+\varepsilon_{f}
$$

where $\varepsilon_{f} \sim \mathscr{N}\left(0, \sigma_{\varepsilon}^{2}\right)$. Using maximum likelihood, we can estimate the values of $\mu$ and the error variance, $\sigma_{\varepsilon}^{2}$, that can best account for the observed adoption responses in both experiments. ${ }^{73}$ Panel A of Table 11 reports these estimates $\hat{\mu}$ and $\hat{\sigma}_{\varepsilon}^{2}$ under six priors ranging from $P=0.01$ to $P=1$. With these estimates in hand, we can ask: what is the ITT estimate we would obtain from a US $\$ 320$ subsidy on adoption, the size of the payment in Experiment 2 ? To answer this question we use $\hat{\mu}$ and $\hat{\sigma}_{\varepsilon}^{2}$ to simulate fixed cost draws 1,000 times, with Figure A.15 displaying the full distribution of Group A firms switching from non-adoption to adoption as a result of a US $\$ 320$ payment. In Panel B of Table 11, we report the average ITT estimate and the standard deviation from these simulations. The ITT estimates range between 0.01 and 0.10 for priors between $P=1$ and $P=0.05$, much lower than the 0.32 we obtained in our incentive-payment experiment (see Panel A of Table 8, Column 3). ${ }^{74}$ Even for the most pessimistic prior of $P=0.01$, we only obtain an ITT of 0.24 . Although such a low prior is theoretically possible, it seems unrealistically pessimistic

\footnotetext{
${ }^{73}$ We use the short-run liberal adoption rates for this exercise. Let adopt $t_{1 f}$ denote an indicator if firm $f$ had adopted the technology in Experiment 1, and adopt $_{2 f}$ an analogous indicator for adoption after Experiment 2. The $\log$ likelihood function for Group A firms is:

$$
\begin{aligned}
L\left(\mu, \sigma_{\varepsilon}\right)= & \sum_{f}\left\{\left(1-\text { adopt }_{1 f}\right)\left(\text { adopt }_{2 f}\right) \ln \left[\Phi\left(\frac{\ln \left[P\left(\frac{N V B_{f}}{0.0187}\right)+320\right]-\mu}{\sigma_{\varepsilon}}\right)-\Phi\left(\frac{\ln \left[P\left(\frac{N V B_{f}}{0.0187}\right)\right]-\mu}{\sigma_{\varepsilon}}\right)\right]\right. \\
& +\left(1-\text { adopt }_{1 f}\right)\left(1-\text { adopt }_{2 f}\right) \ln \left[1-\Phi\left(\frac{\ln \left[P\left(\frac{N V B_{f}}{0.0187}\right)+320\right]-\mu}{\sigma_{\varepsilon}}\right)\right] \\
& \left.+\left(\text { adopt }_{1 f}\right) \ln \left[\Phi\left(\frac{\ln \left[P\left(\frac{N V B_{f}}{0.0187}\right)\right]-\mu}{\sigma_{\varepsilon}}\right)\right]\right\}
\end{aligned}
$$
}

The first expression on the right-hand-side of (3) captures the contribution to the likelihood of initial non-adopter Group-A firms who are induced to switch from non-adoption to adoption because of the $\$ 320$ subsidy. The second expression captures initial non-adopter Group-A firms who are not induced to switch adoption status. The third expression is the contribution of initial adopter firms. For Group-B firms, the likelihood function is the same except adopt $_{2 f}=0 \forall f$ and there is no US $\$ 320$ payment inside the argument of the normal CDF, $\Phi($.$) . We pool both groups$ when maximizing the likelihood function.

${ }^{74}$ Panel $\mathrm{C}$ of Table 11 reports the corresponding probabilities that five or more Group A firms adopt in the short-run (recall that the incentive-payment treatment induced five adopters according to the liberal adoption measure). The probability of observing an increase in adoption of that magnitude is exceedingly low, between 0.000 and 0.013 , for all but the most pessimistic prior of $P=0.01$. 
given the nature of the technology. ${ }^{75}$ In summary, it appears that if unobserved fixed costs are the reason for low adoption in Experiment 1, a US $\$ 320$ subsidy alone cannot plausibly explain the magnitude of the increase in adoption we observed in Experiment 2.

\subsection{Salience}

A second alternative explanation is that the incentive-payment treatment increased the salience of the new technology and that this itself led firms to adopt, independent of any effects on information flows within the firm. There are two variants of this explanation. One variant is that the reminder about the technology during the incentive-intervention visit itself "nudged" firms into adoption. This variant seems an unlikely explanation. We gave the same reminder to the control firms for the incentive intervention (Group B firms) and we saw no firms adopt in the next six months as a result. Also, it is worth noting that we visited all of the tech-drop firms multiple times in our survey rounds. During each of these visits, we discussed the new technology with them and if they were not using it we asked why.

A subtler variant of the salience story is that by putting more money on the table we sent a stronger signal about our own beliefs about the efficacy of our technology, and that this in turn led firms to update their priors about the technology, inducing some firms to adopt. While this explanation is sufficiently flexible that it is difficult to dismiss definitively, it also seems unlikely to have played a major role. We believe that it was clear to firms from the outset, in the initial technology-drop implementation, that we thought that the technology was effective. We then returned to each firm numerous times and in the case of the tech-drop firms discussed the offset die each time. The amount of money we spent on surveying each firm far exceeded the US\$320 payment in the incentive intervention. In short, while in retrospect it seems clear that many owners did not believe us when we told them that the technology works, it does not appear that their skepticism was based on their beliefs about how strongly we held our beliefs, or that the US\$320 affected their beliefs about how strongly we held our beliefs. It seems more likely that they simply thought all along that we had insufficient knowledge and experience in the industry, and hence that our (strongly held) confidence in the technology was misplaced.

\section{Conclusion}

This paper has two basic empirical findings. First, despite the evident advantages of the technology we invented, a surprisingly small number of firms have adopted it, even among the set of firms that we gave it to. This is consistent with a long tradition of research that has found diffusion to be slow for many technologies, but given the characteristics of our technology - low fixed costs, minimal required changes to other aspects of the production process, easily measured cost advantages -

\footnotetext{
${ }^{75}$ Recall that we visited each treatment firm and performed a demonstration of the technology in front of the owner. This demonstration involved having the firm's cutter cut using the traditional die and count the pentagons and then cut using the offset die and count the pentagons. The latter was repeated until the cutter achieved 272 pentagons from a sheet. In no case did this require more than three sheets. The nature of the technology is sufficiently transparent that, even if the owners were not convinced of all aspects of the technology, it seems to us implausible that they placed less than 1 percent probability on the fact that the technology worked as we said it did.
} 
the low adoption rate seems particularly puzzling. Second, with a modest change to the incentives of key employees in the firm - very small in monetary terms relative to firms' revenues and the benefits of adoption - we induced a statistically significant increase in adoption. This is consistent with the hypothesis that a misalignment of incentives within the firm is an important barrier to adoption. Although we do not observe all of the communication between employees and owners, it appears that at least one way that employees have resisted the adoption of our new technology is by misinforming owners about the value of the technology. It further appears that the incentive-payment intervention had a significant effect because it induced workers to reveal the benefits of the technology.

A natural question is why many owners did not simply change the payment scheme on their own, to give employees a greater incentive to adopt the new technology. Survey evidence indicates that some firms are not aware of the availability of alternative payment schemes, and that many firms believe that there are non-trivial transaction costs involved in changing contracts, even implicit ones. One would expect owners to weigh any costs of modifying contracts against the expected benefits of adopting new technologies. If owners have low priors that a new technology is beneficial (or that a beneficial new technology will arrive in the future), they may rationally be unwilling to pay even quite small transaction costs. Although it is difficult for us to distinguish empirically between lack of awareness of alternative contracts and stickiness of contracts as explanations for why owners did not change payment schemes, the key point is that many owners did not in fact change schemes and this left scope for our very modest incentive intervention to have a large effect on adoption.

Our study carried out a particular intervention with a particular technology in a particular sector. This naturally raises questions about external validity. For example, both of our experiments were relatively "hands-off": we gave limited advice to firms after the initial demonstration in the first experiment, or the incentive payment in the second. Would organizational barriers still hinder adoption if the innovators (or their salespeople) had financial incentives to work with firms to promote adoption? We strongly suspect so, since there would still be costs associated with overcoming such barriers, and it is not clear that innovators' incentives would be strong enough to induce them to pay such costs for firms, or that the firms themselves would be willing ex ante to grant innovators the access and time to identify the barriers and resolve them (especially if they receive negative feedback about the technology from their own workers). ${ }^{76}$ Nevertheless, we recognize that more research is required, in other sectors, with other technologies, to determine to what extent our findings in Sialkot can be generalized.

With that caveat, we believe that three implications of our study are likely to apply more broadly. First, we have provided reasonably direct evidence of a complementarity, in the sense of Milgrom and Roberts (1990, 1995), between a technological innovation (the offset die) and an organizational innovation (conditional wage contracts). We suspect that similar complementarities between technical and organizational innovations exist in many other settings, and that it is common for technology adoption to require organizational changes in order to be successful.

\footnotetext{
${ }^{76}$ We also believe that our results are directly relevant to a number of real-world settings in which innovations are developed by non-profits and public-sector entities (e.g. agricultural extension services) that have weak financial incentives but that nonetheless aim to promote adoption of beneficial new technologies.
} 
Second, there appears to be a form of inertia in employment relationships that can hinder technological change. We have argued that firms' choices of labor contracts depend on the rate at which beneficial new technologies are expected to arrive. It also appears that labor contracts, once established, are difficult to modify. The implication is that firms and industries that evolve in technologically stable environments may be less able to adapt to technological change than new firms and industries, or firms and industries that evolve in technologically dynamic environments. Simple piece-rate contracts may well have been optimal for firms in Sialkot before we showed up, but the very fact that firms in Sialkot have been producing for decades using them may itself have contributed to low adoption rates of the offset die. ${ }^{77}$

Finally, it seems likely that for technology adoption to be successful employees need to have an expectation that they will share in the gains from adoption. Such an expectation may be generated by a variety of different types of contracts, implicit or explicit. But to the extent that firms must rely on the knowledge of shopfloor workers about the value of new technologies or how best to implement them, it appears to be important that some sort of credible gain-sharing mechanism be in place.

\section{References}

Aghion, P., And J. Tirole (1997): "Formal and Real Authority in Organizations," Journal of Political Economy, 105(1), 1-29.

Anderson, S. T., And R. G. Newell (2004): "Information Programs for Technology Adoption: The Case of Energy-Efficiency Audits," Resource and Energy Economics, 26(1), 27 - 50.

Andridge, R. R., And R. A. Little (2010): "A Review of Hot Deck Imputation for Survey Non-response," International Statistics Review, 78(1), 40-64.

Atkin, D., A. Chaudhry, S. Chaudry, A. K. Khandelwal, T. Raza, and E. Verhoogen (2016): "On the Origins and Development of Pakistan's Soccer-Ball Cluster," World Bank Economic Review (ABCDE Conference Papers \& Proceedings).

Atkin, D., A. Chaudhry, S. Chaudry, A. K. Khandelwal, and E. Verhoogen (2015): "Mark-up and Cost Dispersion across Firms: Direct Evidence from Producer Surveys in Pakistan," American Economic Review Papers 83 Proceedings, 105(5), 537-544.

Bandiera, O., I. Barankay, and I. Rasul (2005): "Social Preferences and the Response to Incentives: Evidence from Personnel Data," Quarterly Journal of Economics, 120(3), 917-962.

- (2007): "Incentives for Managers and Inequality among Workers: Evidence from a Firm-Level Experiment," Quarterly Journal of Economics, 122(2), 729-773.

- (2009): "Social Connections and Incentives in the Workplace: Evidence from Personnel Data," Econometrica, 77(4), 1047-1094.

- (2011): "Field Experiments with Firms," Journal of Economic Perspectives, 25(3), 63-82.

Bandiera, O., AND I. Rasul (2006): "Social Networks and Technology Adoption in Northern Mozambique," Economic Journal, 116(514), 869 - 902.

Beaman, L., A. Ben-Yishay, J. Magruder, and A. M. Mobarak (2015): "Can Network Theory-based Targeting Increase Technology Adoption?," Unpub. paper, Northwestern Univ.

Beaman, L., J. Magruder, and J. Robinson (2014): "Minding Small Change among Small Firms in Kenya," Journal of Development Economics, 108, 69 - 86.

\footnotetext{
${ }^{77}$ The literature on organizational failures reviewed by Garicano and Rayo (2016) has focused on major technical disruptions. One contribution of this paper, in addition to providing experimental evidence in a many-firm setting, is to show that firms have difficulty adapting even to incremental technological improvements. While the foregone surplus is modest in this case, it seems plausible that such mini-failures are common in organizations, and that their cumulative effect is substantial.
} 
BenYishay, A., And A. M. Mobarak (2014): "Social Learning and Communication," NBER working paper no. 20139.

Bloom, N., B. Eifert, A. Mahajan, D. McKenzie, and J. Roberts (2013): "Does Management Matter? Evidence from India," Quarterly Journal of Economics, 128(1), 1-51.

Bloom, N., And J. Van Reenen (2007): "Measuring and Explaining Management Practices Across Firms and Countries," Quarterly Journal of Economics, 122(4), 1351-1408.

- (2010): "Why Do Management Practices Differ across Firms and Countries?," Journal of Economic Perspectives, 24(1), 203-24.

Bresnahan, T. F., and M. Trajtenberg (1995): "General Purpose Technologies: 'Engines of Growth'?," Journal of Econometrics, 65(1), 83 - 108.

Carmichael, H. L., And W. B. MacLeod (2000): "Worker Cooperation and the Ratchet Effect," Journal of Labor Economics, 18(1), 1-19.

Chen, Y., N. Kartik, And J. Sobel (2008): "Selecting Cheap-Talk Equilibria," Econometrica, 76(1), $117-136$.

Clawson, D. (1980): Bureaucracy and the Labor Process: The Transformation of US Industry, 1860-1920. Monthly Review Press New York.

Cohen, J., And P. Dupas (2010): "Free Distribution or Cost-Sharing? Evidence from a Randomized Malaria Prevention Experiment," Quarterly Journal of Economics, 125(1), 1-45.

Coleman, James, E. K., And H. Menzel (1966): Medical Innovation: A Diffusion Study. Bobbs-Merrill, New York.

Conley, T., And C. Udry (2010): "Learning about a New Technology: Pineapple in Ghana," American Economic Review, 100(1), 35-69.

Crawford, V. P., ANd J. Sobel (1982): "Strategic Information Transmission," Econometrica, 50(6), 1431-1451.

David, P. A. (1990): "The Dynamo and the Computer: An Historical Perspective on the Modern Productivity Paradox," American Economic Review Papers and Proceedings, 80(2), 355-61.

de Mel, S., D. J. McKenzie, and C. Woodruff (2008): "Returns to Capital in Microenterprises: Evidence from a Field Experiment," Quarterly Journal of Economics, 123(4), 1329-1372.

Dearden, J., B. W. Ickes, and L. Samuelson (1990): "To Innovate or Not to Innovate: Incentives and Innovation in Hierarchies," American Economic Review, 80(5), 1105-1124.

Dessein, W. (2002): "Authority and Communication in Organizations," Review of Economic Studies, $69(4), 811-838$.

Dow, J., And E. Perotti (2013): "Resistance to Change," Unpub. paper, London Business School.

Duflo, E., M. Kremer, And J. Robinson (2011): "Nudging Farmers to Use Fertilizer: Theory and Experimental Evidence from Kenya," American Economic Review, 101(6), 2350-2390.

Dupas, P. (2014): "Short-run Subsidies and Long-Run Adoption of New Health Products: Evidence from a Field Experiment," Econometrica, 82(1), 197-228.

EDWARds, R. C. (1979): Contested Terrain: The Transformation of the Workplace in the Twentieth Century. Basic Books.

Emerick, K., A. de Janvry, E. Sadoulet, and M. H. Dar (2016): "Technological Innovations, Downside Risk, and the Modernization of Agriculture," American Economic Review, 106(6), 1537-61.

Foster, A. D., And M. R. Rosenzweig (1995): "Learning by Doing and Learning from Others: Human Capital and Technical Change in Agriculture," Journal of Political Economy, 103(6), 1176-1209.

Fowlie, M., M. Greenstone, and C. Wolfram (2015a): "Are the Non-monetary Costs of Energy Efficiency Investments Large? Understanding Low Take-Up of a Free Energy Efficiency Program," American Economic Review, 105(5), 201-04.

- (2015b): "Do Energy Efficiency Investments Deliver? Evidence from the Weatherization Assistance Program," Unpub. paper, UC Berkeley.

Freeman, R. B., AND M. M. Kleiner (2005): "The Last American Shoe Manufacturers: Decreasing Productivity and Increasing Profits in the Shift from Piece Rates to Continuous Flow Production," Industrial Relations: A Journal of Economy and Society, 44(2), 307-330.

Garicano, L., And L. Rayo (2016): "Why Organizations Fail: Models and Cases," Journal of Economic 
Literature, 54(1), 137-192.

Geroski, P. A. (2000): "Models of Technology Diffusion," Research Policy, 29(4-5), 603 - 625.

Gibbons, R. (1987): "Piece-Rate Incentive Schemes," Journal of Labor Economics, pp. 413-429.

Gibbons, R., And J. Roberts (2013): "Economic Theories of Incentives in Organizations," in Handbook of Organizational Economics, ed. by R. Gibbons, and J. Roberts. Princeton: Princeton University Press.

Griliches, Z. (1957): "Hybrid Corn: An Exploration in the Economics of Technological Change," Econometrica, 25(4), 501-22.

Hall, B. (2005): "Innovation and Diffusion," in Oxford Handbook of Innovation, ed. by J. Fagerberg, D. C. Mowery, and R. R. Nelson, pp. 459-484. Oxford University Press.

Hall, B., And B. Khan (2003): "Adoption of New Technology," in New Economy Handbook, ed. by D. C. Jones. Academic Press, San Diego.

Hanna, R., S. Mullainathan, and J. Schwartzstein (2014): "Learning Through Noticing: Theory and Experimental Evidence in Farming," Quarterly Journal of Economics, 129(3), 1311-1353.

Hardy, M., And J. McCasland (2016): "It Takes Two: Experimental Evidence on the Determinants of Technology Diffusion," Unpub. paper, University of British Columbia.

Holmstrom, B., And P. Milgrom (1991): "Multi-Task Principal-Agent Analyses: Incentive Contracts, Asset Ownership and Job Design," Journal of Law, Economics and Organization, 7, 24-52.

Ichniowski, C., AND K. Shaw (2013): "Insider Econometrics," in Handbook of Organizational Economics, ed. by R. Gibbons, and J. Roberts. Princeton: Princeton University Press.

Khan, A. Q., A. Khwaja, And B. Olken (2016): "Tax Farming Redux: Experimental Evidence on Incentive Pay for Tax Collectors," Quarterly Journal of Economics, 131(1), 219-271.

Khan, F. R., K. A. Munir, And H. Willmott (2007): "A Dark Side of Institutional Entrepreneurship: Soccer Balls, Child Labour and Postcolonial Impoverishment," Organization Studies, 28(7), 1055-1077.

Krishna, V., AND J. Morgan (2008): "Contracting for Information under Imperfect Commitment," RAND Journal of Economics, 39(4), 905-925.

Kuperberg, G., And W. Kuperberg (1990): "Double-Lattice Packings of Convex Bodies in the Plane," Discrete $\mathcal{E}$ Computational Geometry, 5, 389-397.

Lazear, E. P. (1986): "Salaries and Piece Rates," Journal of Business, 59(3), 405-431.

Lazear, E. P., AND P. Oyer (2013): "Personnel Economics," in Handbook of Organizational Economics, ed. by R. Gibbons, and J. Roberts. Princeton: Princeton University Press.

Lazonick, W. (1979): "Industrial Relations and Technical Change: The Case of the Self-Acting Mule," Cambridge Journal of Economics, 3(3), 231-262.

(1990): Competitive Advantage on the Shop Floor. Cambridge MA: Harvard University Press.

Lefley, F. (1996): "The Payback Method of Investment Appraisal: A Review and Synthesis," International Journal of Production Economics, 44(3), 207 - 224.

Mansfield, E. (1961): "Technical Change and the Rate of Imitation," Econometrica, 29(4), 741-766.

(1989): "The Diffusion of Industrial Robots in Japan and the United States," Research Policy, $18(4), 183-192$.

Marglin, S. A. (1974): "What Do Bosses Do?," Review of Radical Political Economy, 6(2), 60-112.

Mathewson, S. B. (1931): Restriction of Output among Unorganized Workers. Viking Press: New York.

Miguel, E., And M. Kremer (2004): "Worms," Econometrica, 72(1), 159-217.

Milgrom, P., And J. Roberts (1990): "The Economics of Modern Manufacturing: Technology, Strategy and Organization," American Economic Review, 80(3), 511-528.

(1995): "Complementarities and Fit: Strategy, Structure and Organizational Change in Manufacturing," Journal of Accounting and Economics, 19, 179-208.

MokyR, J. (1990): The Lever of Riches. Oxford University Press.

Munshi, K. (2004): "Social Learning in a Heterogeneous Population: Technology Adoption in the Indian Green Revolution," Journal of Development Economics, 73, 185-213.

Rosenberg, N. (1972): "Factors Affecting the Diffusion of Technology," Explorations in Economic History, 10(1), $3-33$.

— (1982): Inside the Black Box: Technology and Economics. Cambridge UK: Cambridge University 
Press.

Roy, D. (1952): "Quota Restriction and Goldbricking in a Machine Shop," American Journal of Sociology, $57,427-442$.

Ryan, B., And N. C. Gross (1943): "Diffusion of Hybrid Seed Corn in Two Iowa Communities," Rural Sociology, 8, 15-24.

Stole, L. A., And J. Zwiebel (1996): "Organizational Design and Technology Choice under Intrafirm Bargaining," American Economic Review, 86(1), 195-222.

Suri, T. (2011): "Selection and Comparative Advantage in Technology Adoption," Econometrica, 79(1), 159-209.

Syverson, C. (2011): "What Determines Productivity?," Journal of Economic Literature, 49(2), 326-65.

TAYlor, F. W. (1896): "A Piece-Rate System," Economic Studies, 1(2), 89-129.

- (1911): The Principles of Scientific Management. Harper \& Brothers: New York and London. 
Figure 1: Traditional 2-Hexagon and 2-Pentagon Dies

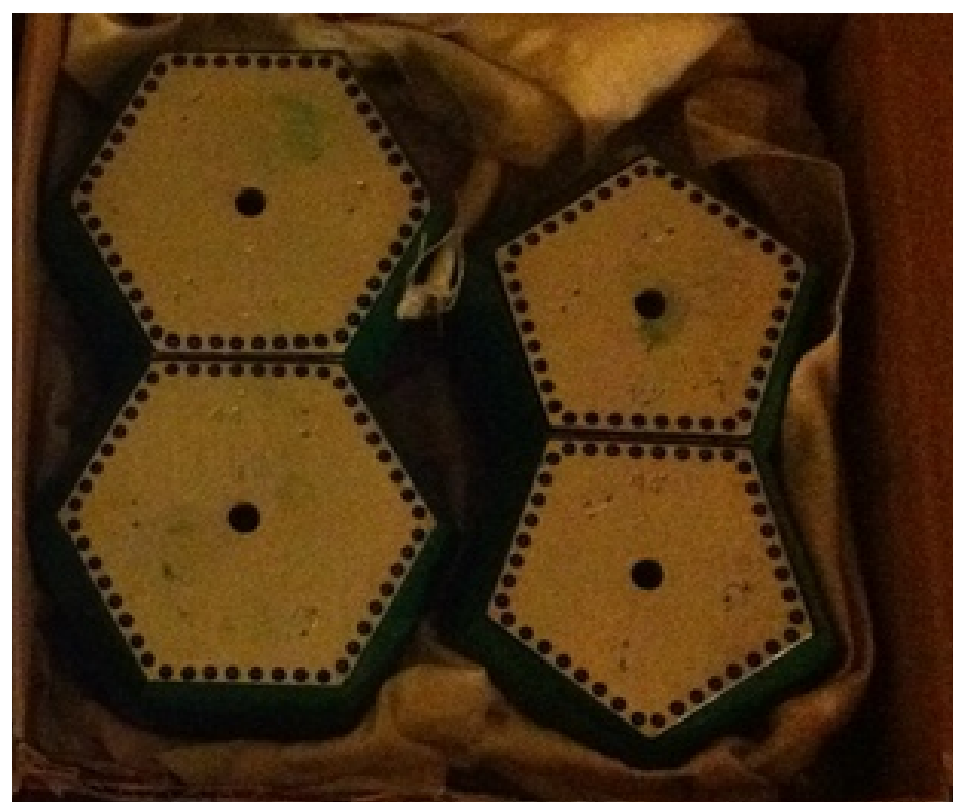

Figure 2: Laminated Rexine Wastage, Hexagons

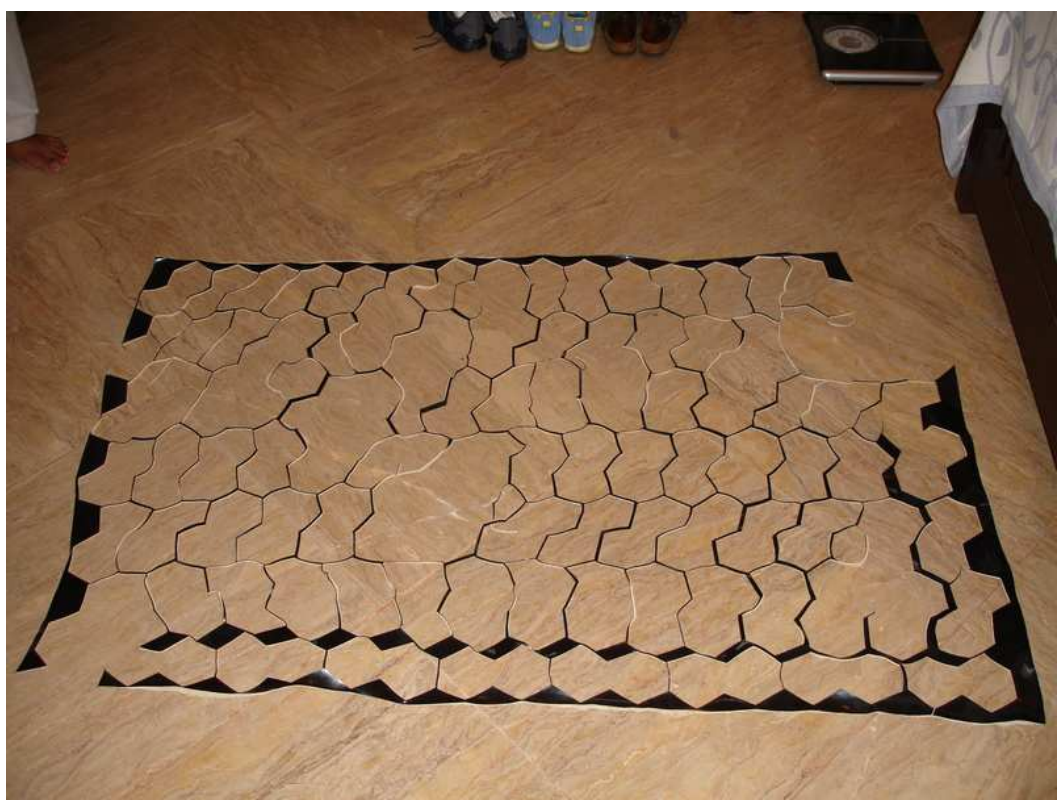

Notes: Figure displays laminated rexine wastage from cutting hexagons with the traditional two-hexagon die. 
Figure 3: Laminated Rexine Wastage, Pentagons, Traditional Die

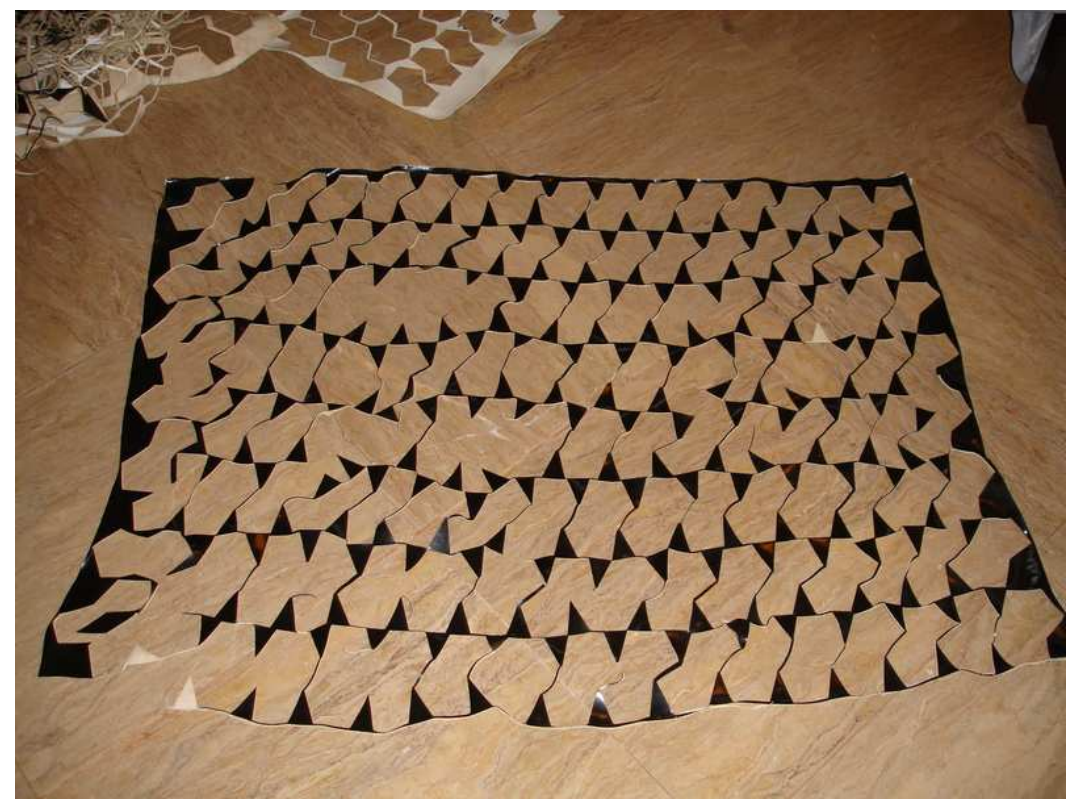

Notes: Figure displays laminated rexine wastage from cutting pentagons with the traditional two-pentagon die.

Figure 4: Cutting Pattern and Blueprint for "Offset" Four-Pentagon Die

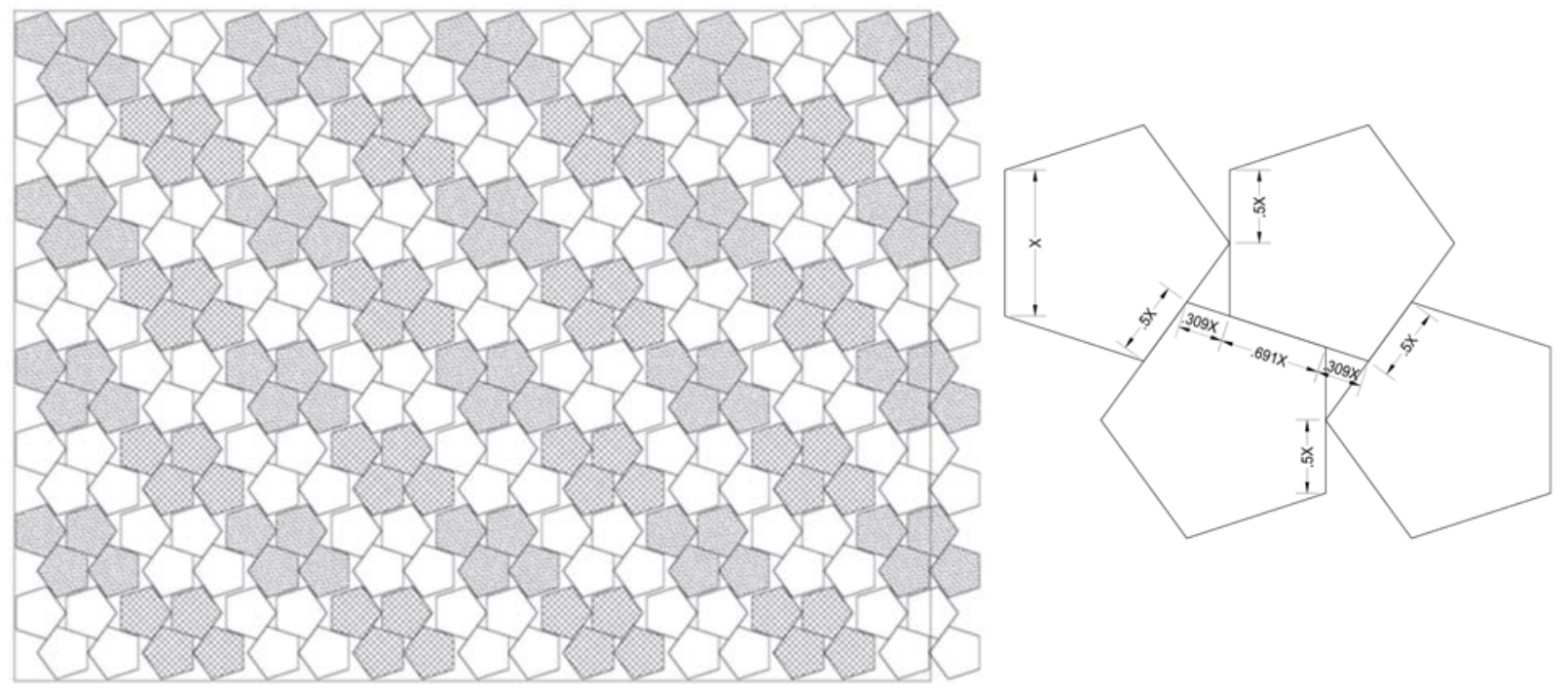

Notes: Left figure displays the cutting pattern for the four-panel offset die. Right figure displays blueprint of the four-panel offset die that was provided to Tech-Drop firms. The blueprint contained instructions for modifying the size of the die. 


\section{Figure 5: Implied Bounds on Fixed Costs Under a Learning Subsidy Explanation}

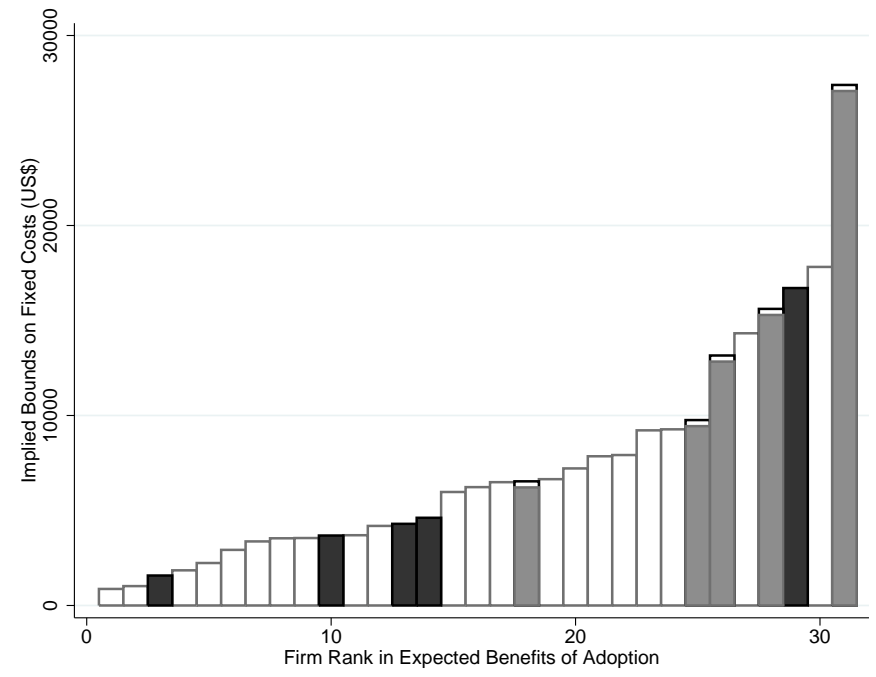

Notes: Figure displays implied bounds on fixed costs for each firm in incentive-payment intervention, assuming a 25 percent per year interest rate and a prior of 0.5 that the technology works (see Section 8.1). Solid black bars indicate upper bounds for the initial adopters, and white bars indicate lower bounds for the never-adopters. Solid grey bars indicate lower bounds for initial non-adopters that adopted in response to the incentive treatment (Experiment-2 compliers); black outlines above the solid grey bars indicate upper bounds for these firms. (Unobserved fixed costs can simultaneously explain both initial non-adoption and subsequent adoption for these firms only if they fall between top of grey bar and black outline.) 
Table 1: Pentagons per Sheet

\begin{tabular}{|c|c|c|c|c|}
\hline & \multicolumn{2}{|c|}{ traditional die } & \multicolumn{2}{|c|}{ offset die } \\
\hline & $\begin{array}{c}\text { owner report } \\
(1)\end{array}$ & $\begin{array}{c}\text { direct obs. } \\
(2)\end{array}$ & $\begin{array}{c}\text { owner report } \\
(3)\end{array}$ & $\begin{array}{c}\text { direct obs. } \\
(4)\end{array}$ \\
\hline size 43.5 & $\begin{array}{l}257.3 \\
(10.6)\end{array}$ & $\begin{array}{c}257.7 \\
(6.7)\end{array}$ & $\begin{array}{c}273.5 \\
(4.4)\end{array}$ & $\begin{array}{c}277.5 \\
(5.3)\end{array}$ \\
\hline size 43.75 & $\begin{array}{l}256.3 \\
(6.7)\end{array}$ & $\begin{array}{l}254.4 \\
(9.4)\end{array}$ & $\begin{array}{l}269.0 \\
(1.4)\end{array}$ & $\begin{array}{c}272.0 \\
(0.0)\end{array}$ \\
\hline size 44 & $\begin{array}{c}254.3 \\
(9.1)\end{array}$ & $\begin{array}{c}248.4 \\
(18.7)\end{array}$ & 280.0 & $\begin{array}{c}272.5 \\
(0.7)\end{array}$ \\
\hline size 44.25 & $\begin{array}{c}246.1 \\
(8.3)\end{array}$ & 262.0 & 272.0 & \\
\hline rescaled (to size 44 ) & $\begin{array}{c}254.2 \\
(8.9)\end{array}$ & $\begin{array}{c}248.3 \\
(11.0)\end{array}$ & $\begin{array}{c}280.0 \\
(3.0)\end{array}$ & $\begin{array}{c}275.4 \\
(4.8)\end{array}$ \\
\hline $\mathrm{N}$ (all sizes) & 320 & 39 & 8 & 10 \\
\hline
\end{tabular}

Notes: Table reports average (non-defective) pentagons per sheet by die size. Column 1 indicates self-reported numbers from the owner, several rounds per firm in some cases. Column 2 indicates pentagons per sheet directly observed by the survey team for tech-drop firms (during the initial cutting demonstration) and for cash-drop firms (at the time of the cash drop). Columns 3-4 report numbers for the offset die and were only collected from tech-drop firms. In the fifth row, pentagons per sheet are rescaled using means for each size in each column. The final row reports the pooled number of observations for all die sizes. Standard deviations in parentheses. 
Table 2: Benefits from Adopting the Offset Die

\begin{tabular}{|c|c|c|c|c|c|c|}
\hline & $10^{t h}$ & $25^{t h}$ & $50^{t h}$ & $75^{\text {th }}$ & $90^{t h}$ & mean \\
\hline \multicolumn{7}{|c|}{ A. Variable cost reduction from reduced waste of material } \\
\hline reduction in pentagon laminated rexine cost (\%) & $\begin{array}{c}4.24 \\
(0.99)\end{array}$ & $\begin{array}{c}5.56 \\
(0.85)\end{array}$ & $\begin{array}{c}6.76 \\
(0.59)\end{array}$ & $\begin{array}{c}8.02 \\
(1.59)\end{array}$ & $\begin{array}{l}10.15 \\
(2.10)\end{array}$ & $\begin{array}{c}6.93 \\
(0.70)\end{array}$ \\
\hline laminated rexine as share of total cost (\%) & $\begin{array}{l}34.51 \\
(3.06)\end{array}$ & $\begin{array}{l}39.80 \\
(1.86)\end{array}$ & $\begin{array}{l}44.83 \\
(1.94)\end{array}$ & $\begin{array}{l}51.30 \\
(1.72)\end{array}$ & $\begin{array}{l}56.09 \\
(3.84)\end{array}$ & $\begin{array}{l}45.82 \\
(1.58)\end{array}$ \\
\hline variable cost reduction (\%) & $\begin{array}{c}0.59 \\
(0.11)\end{array}$ & $\begin{array}{c}0.77 \\
(0.10)\end{array}$ & $\begin{array}{c}0.98 \\
(0.09)\end{array}$ & $\begin{array}{c}1.24 \\
(0.19)\end{array}$ & $\begin{array}{c}1.62 \\
(0.27)\end{array}$ & $\begin{array}{c}1.05 \\
(0.11)\end{array}$ \\
\hline \multicolumn{7}{|c|}{ B. Variable cost increase from increased labor time } \\
\hline cutter wage as share of cost $(\%)$ & $\begin{array}{c}0.30 \\
(0.03)\end{array}$ & $\begin{array}{c}0.36 \\
(0.01)\end{array}$ & $\begin{array}{c}0.45 \\
(0.02)\end{array}$ & $\begin{array}{c}0.59 \\
(0.03)\end{array}$ & $\begin{array}{c}0.71 \\
(0.04)\end{array}$ & $\begin{array}{c}0.48 \\
(0.02)\end{array}$ \\
\hline variable cost increase $(\%)$ & $\begin{array}{c}0.10 \\
(0.01)\end{array}$ & $\begin{array}{c}0.12 \\
(0.00)\end{array}$ & $\begin{array}{c}0.15 \\
(0.01)\end{array}$ & $\begin{array}{c}0.19 \\
(0.01)\end{array}$ & $\begin{array}{c}0.23 \\
(0.01)\end{array}$ & $\begin{array}{c}0.16 \\
(0.01)\end{array}$ \\
\hline C. Net benefits & & & & & & \\
\hline net variable cost reduction (\%) & $\begin{array}{c}0.42 \\
(0.11)\end{array}$ & $\begin{array}{c}0.61 \\
(0.10)\end{array}$ & $\begin{array}{c}0.82 \\
(0.09)\end{array}$ & $\begin{array}{l}1.09 \\
(0.19)\end{array}$ & $\begin{array}{c}1.47 \\
(0.27)\end{array}$ & $\begin{array}{c}0.89 \\
(0.11)\end{array}$ \\
\hline$\%$ net variable cost/avg $\%$ profit rate & $\begin{array}{c}4.55 \\
(1.05)\end{array}$ & $\begin{array}{c}6.82 \\
(1.13)\end{array}$ & $\begin{array}{l}10.63 \\
(1.60)\end{array}$ & $\begin{array}{l}16.56 \\
(2.35)\end{array}$ & $\begin{array}{l}24.42 \\
(4.15)\end{array}$ & $\begin{array}{l}13.07 \\
(1.79)\end{array}$ \\
\hline total cost savings per month (Rs 000s) & $\begin{array}{c}3.66 \\
(0.99)\end{array}$ & $\begin{array}{c}9.82 \\
(2.33)\end{array}$ & $\begin{array}{l}41.35 \\
(9.43)\end{array}$ & $\begin{array}{l}135.92 \\
(36.39)\end{array}$ & $\begin{array}{c}397.95 \\
(130.62)\end{array}$ & $\begin{array}{l}137.77 \\
(31.68)\end{array}$ \\
\hline total cost savings per cutter per month (Rs 000s) & $\begin{array}{c}2.75 \\
(0.83)\end{array}$ & $\begin{array}{c}6.47 \\
(1.33)\end{array}$ & $\begin{array}{l}14.91 \\
(2.43)\end{array}$ & $\begin{array}{c}33.83 \\
(6.28)\end{array}$ & $\begin{array}{c}63.61 \\
(14.02)\end{array}$ & $\begin{array}{l}27.31 \\
(5.04)\end{array}$ \\
\hline days to recover fixed costs & $\begin{array}{l}10.28 \\
(2.23)\end{array}$ & $\begin{array}{l}19.11 \\
(3.66)\end{array}$ & $\begin{array}{l}43.03 \\
(7.37)\end{array}$ & $\begin{array}{l}100.86 \\
(21.74)\end{array}$ & $\begin{array}{l}247.53 \\
(76.42)\end{array}$ & $\begin{array}{l}168.80 \\
(84.72)\end{array}$ \\
\hline days to recover fixed costs (no die) & $\begin{array}{c}5.34 \\
(1.16)\end{array}$ & $\begin{array}{c}9.92 \\
(1.90)\end{array}$ & $\begin{array}{l}22.34 \\
(3.83)\end{array}$ & $\begin{array}{c}52.37 \\
(11.29)\end{array}$ & $\begin{array}{l}128.53 \\
(39.68)\end{array}$ & $\begin{array}{c}87.64 \\
(43.99)\end{array}$ \\
\hline
\end{tabular}

Notes: Each row reports the distribution across firms of indicated variable. $1^{\text {st }}$ row: percentage reduction in per-pentagon laminated rexine cost from adopting the offset die. $2^{\text {nd }}$ row: laminated rexine cost as a percentage of total unit costs. $3^{\text {rd }}$ row: variable cost reduction due to reduced waste of material, computed as product of reduction in per-pentagon laminated rexine cost times $33 \%$ (share of pentagons relative to hexagons in total laminated rexine costs) times laminated rexine's share of total cost. $4^{\text {th }}$ row: cutter's wage as a share of unit costs. $5^{\text {th }}$ row: variable cost increase due to increased labor time, calculated as cutter wage share of cost times 33\% (pentagon share of total laminated rexine cost) times $100 \%$ (conservative assumption of increase in cutting time). $6^{\text {th }}$ row: net variable cost of reduction, i.e. difference between variable cost reduction due to reduced wages and variable cost increase due to increased labor time. $7^{\text {th }}$ row: ratio of the net variable cost reduction to firm's average profit margin. $8^{\text {th }}$ row: total cost savings per month in Rupees (exchange rate Rs $100=\mathrm{US} \$ 1$ ). $9^{\text {th }}$ row: cost savings per month per cutter in Rupees. $10^{\text {th }}$ row: days needed to recover all fixed costs of adoption. $11^{\text {th }}$ row: days needed to recover fixed costs of adoption, excluding purchase of die (relevant for tech-drop firms which received die for free). Table uses hot-deck imputation procedure to replace missing values with draws from distribution of non-missing values for firm's stratum. Entire procedure bootstrapped 1,000 times; quantiles and mean of each variable calculated for each iteration. Table reports mean and standard deviation for each statistic and variable. Details in Section 2.4. 
Table 3: Firm Characteristics by Quantile

\begin{tabular}{|c|c|c|c|c|c|c|c|c|c|}
\hline & Mean & Min & $10^{t h}$ & $25^{t h}$ & $50^{t h}$ & $75^{\text {th }}$ & $90^{t h}$ & Max & $\mathrm{N}$ \\
\hline \multicolumn{10}{|c|}{ A. Initial-responder sample } \\
\hline avg output/month (000s) & 31.2 & 0.8 & 1.5 & 3.2 & 10.0 & 34.1 & 71.7 & 275.0 & 85 \\
\hline avg employment & 89.8 & 4.0 & 5.6 & 7.4 & 18.3 & 50.0 & 235.0 & $1,700.0$ & 85 \\
\hline avg employment (cutters) & 5.6 & 0.7 & 1.0 & 1.0 & 2.2 & 5.0 & 12.0 & 123.0 & 85 \\
\hline avg Rs/ball (head cutter) & 1.5 & 1.0 & 1.2 & 1.3 & 1.5 & 1.6 & 1.9 & 2.9 & 79 \\
\hline avg $\%$ size 5 & 89.4 & 52.4 & 68.1 & 84.0 & 94.0 & 100.0 & 100.0 & 100.0 & 85 \\
\hline $\operatorname{avg} \%$ size 4 & 3.1 & 0.0 & 0.0 & 0.0 & 0.0 & 4.1 & 7.8 & 35.0 & 85 \\
\hline avg $\%$ size other & 7.5 & 0.0 & 0.0 & 0.0 & 1.7 & 11.2 & 20.0 & 47.6 & 85 \\
\hline avg $\%$ promotional (of size 5 ) & 41.4 & 0.0 & 2.0 & 18.8 & 41.1 & 62.4 & 80.0 & 100.0 & 85 \\
\hline avg price, size 5 promotional & 243.1 & 152.5 & 185.0 & 200.0 & 231.2 & 269.1 & 300.0 & 575.0 & 64 \\
\hline avg price, size 5 training & 441.3 & 200.0 & 275.0 & 316.7 & 392.5 & 490.0 & 600.0 & $2,250.0$ & 73 \\
\hline avg profit $\%$, size 5 promo & 8.1 & 2.6 & 3.9 & 5.0 & 7.9 & 10.2 & 12.5 & 20.0 & 64 \\
\hline avg profit $\%$, size 5 training & 7.9 & 1.6 & 3.2 & 4.6 & 7.8 & 9.9 & 11.8 & 22.2 & 71 \\
\hline avg \% lamination in-house & 95.7 & 31.2 & 81.2 & 100.0 & 100.0 & 100.0 & 100.0 & 100.0 & 75 \\
\hline$\%$ standard design (of size 5 ) & 90.7 & 0.0 & 70.0 & 85.0 & 100.0 & 100.0 & 100.0 & 100.0 & 80 \\
\hline age of firm & 25.4 & 2.0 & 6.0 & 12.0 & 19.5 & 36.5 & 54.0 & 108.0 & 84 \\
\hline CEO experience & 17.0 & 3.0 & 6.0 & 9.0 & 15.5 & 22.0 & 28.0 & 66.0 & 82 \\
\hline head cutter experience & 20.5 & 2.0 & 8.0 & 12.0 & 18.5 & 26.5 & 41.0 & 46.0 & 36 \\
\hline head cutter tenure & 11.1 & 0.0 & 2.0 & 6.0 & 9.0 & 15.0 & 22.0 & 46.0 & 35 \\
\hline \multicolumn{10}{|l|}{ B. Full sample } \\
\hline avg output/month (000s) & 33.4 & 0.0 & 1.6 & 4.6 & 15.2 & 37.0 & 86.2 & 275.0 & 116 \\
\hline avg employment & 103.2 & 4.0 & 6.0 & 8.2 & 24.9 & 75.0 & 227.0 & $2,180.0$ & 115 \\
\hline avg employment (cutters) & 5.2 & 0.7 & 1.0 & 1.2 & 2.4 & 5.0 & 12.0 & 123.0 & 115 \\
\hline avg Rs/ball (head cutter) & 1.6 & 1.0 & 1.1 & 1.3 & 1.5 & 1.7 & 2.1 & 3.0 & 108 \\
\hline avg $\%$ size 5 & 88.6 & 42.8 & 64.2 & 83.3 & 94.4 & 100.0 & 100.0 & 100.0 & 115 \\
\hline $\operatorname{avg} \%$ size 4 & 2.5 & 0.0 & 0.0 & 0.0 & 0.0 & 3.3 & 6.0 & 35.0 & 115 \\
\hline avg $\%$ size other & 8.9 & 0.0 & 0.0 & 0.0 & 2.2 & 13.3 & 31.9 & 57.2 & 115 \\
\hline avg $\%$ promotional (of size 5 ) & 37.0 & 0.0 & 0.0 & 8.3 & 33.8 & 55.2 & 80.0 & 100.0 & 114 \\
\hline avg price, size 5 promotional & 248.6 & 150.0 & 185.0 & 205.0 & 236.7 & 270.0 & 310.0 & 575.0 & 83 \\
\hline avg price, size 5 training & 465.3 & 200.0 & 300.0 & 335.2 & 400.0 & 508.2 & 662.5 & $2,250.0$ & 102 \\
\hline avg profit (\%), size 5 promo & 8.1 & 2.6 & 3.9 & 5.0 & 7.4 & 10.4 & 13.6 & 20.0 & 82 \\
\hline avg profit (\%), size 5 training & 8.1 & 1.6 & 3.2 & 5.0 & 8.0 & 10.0 & 13.0 & 22.2 & 98 \\
\hline avg $\%$ lamination in-house & 96.2 & 25.0 & 85.0 & 100.0 & 100.0 & 100.0 & 100.0 & 100.0 & 104 \\
\hline
\end{tabular}

Notes: Variables beginning with "avg ..." represent within-firm averages across all rounds for which responses are available. Initial responder sample contains firms that responded to baseline survey. Piece rate and prices are in Rupees (exchange rate Rs100=US\$1). Size 5 is regulation size for adults; size 4 is commonly used by children; "avg \% size other" refers to sizes 1,2, and 3. Age, experience and tenure in years. In this table, firms with missing values in all rounds in which questions were asked are dropped (hence the variation in $\mathrm{N}$ in final column.) 
Table 4: Treatment Assignment, Tech-Drop Experiment

\begin{tabular}{|c|c|c|c|c|}
\hline & \multicolumn{4}{|c|}{ \# Firms } \\
\hline & Tech Drop & Cash Drop & No Drop & Total \\
\hline \multicolumn{5}{|l|}{ A. Initial responders } \\
\hline smallest & 5 & 3 & 12 & 20 \\
\hline medium-small & 6 & 3 & 13 & 22 \\
\hline medium-large & 6 & 3 & 13 & 22 \\
\hline largest & 6 & 3 & 12 & 21 \\
\hline total & 23 & 12 & 50 & 85 \\
\hline \multicolumn{5}{|l|}{ B. Initial non-responders } \\
\hline active, late response & 12 & 5 & 14 & 31 \\
\hline active, refused all surveys & 0 & 1 & 15 & 16 \\
\hline inactive (revealed not to be a producer) & 7 & 3 & 12 & 22 \\
\hline
\end{tabular}

Notes: Table reports numbers of firms by treatment assignment among initial responders (Panel A) and initial non-responders (Panel B). Active firms are those who had produced soccer balls in the previous 12 months and cut their own laminated rexine. Last row reports numbers of firms included in initial randomization that were believed to be active (based on an initial listing exercise) but were later revealed not to be active by our definition, because they (a) had shifted entirely to other products, (b) had gone out of business, or (c) were not cutting their own laminated rexine. 
Table 5: Adoption of Technology as of August 2013

\begin{tabular}{|c|c|c|c|c|}
\hline & $\begin{array}{l}\text { Tech } \\
\text { Drop }\end{array}$ & $\begin{array}{l}\text { Cash } \\
\text { Drop }\end{array}$ & $\begin{array}{c}\text { No } \\
\text { Drop }\end{array}$ & Total \\
\hline \multicolumn{5}{|l|}{ A. Initial-responder sample } \\
\hline \# ever responded & 23 & 12 & 50 & $\begin{array}{l}85 \\
85\end{array}$ \\
\hline \# currently active and ever responded & 22 & 11 & 46 & 79 \\
\hline \# traded in & 15 & 0 & 0 & 15 \\
\hline \# ordered offset die (beyond trade-in) & 1 & 0 & 4 & 5 \\
\hline \# received offset die (beyond trade-in) & 1 & 0 & 2 & 3 \\
\hline \# ever used offset die (>1000 balls, conservative) & 3 & 0 & 0 & 3 \\
\hline \# ever used offset die (>1000 balls, liberal) & 4 & 0 & 0 & 4 \\
\hline \multicolumn{5}{|l|}{ B. Full sample } \\
\hline \# ever active firms & 35 & 18 & 79 & 132 \\
\hline \# ever responded & 35 & 17 & 64 & 116 \\
\hline \# currently active and ever responded & 32 & 15 & 59 & 106 \\
\hline \# traded in & 19 & 0 & 0 & 19 \\
\hline \# ordered offset die (beyond trade-in) & 1 & 0 & 6 & 7 \\
\hline \# received offset die (beyond trade-in) & 1 & 0 & 4 & 5 \\
\hline \# ever used offset die (>1000 balls, conservative) & 4 & 0 & 1 & 5 \\
\hline \# ever used offset die (>1000 balls, liberal) & 5 & 0 & 1 & 6 \\
\hline
\end{tabular}

Notes: Table reports adoption statistics as of August 2013 in the initial-responder sample (Panel A) and the full sample (Panel B). "\# ever responded" is the number of firms that answered at least one of the surveys across rounds. 4th row reports the number of firms that took up the option to trade in the original 4-panel offset die for a different offset die. The discrepancy between the 5 th and 6 th rows is due to one diemaker being very slow in delivering offset dies and firms canceling their orders. 7th row uses "conservative" definition of adoption: the number of firms that produced at least 1,000 balls as of August 2013 based on survey data. 8th row uses "liberal" definition, which combines survey data and field reports from our enumerators. 


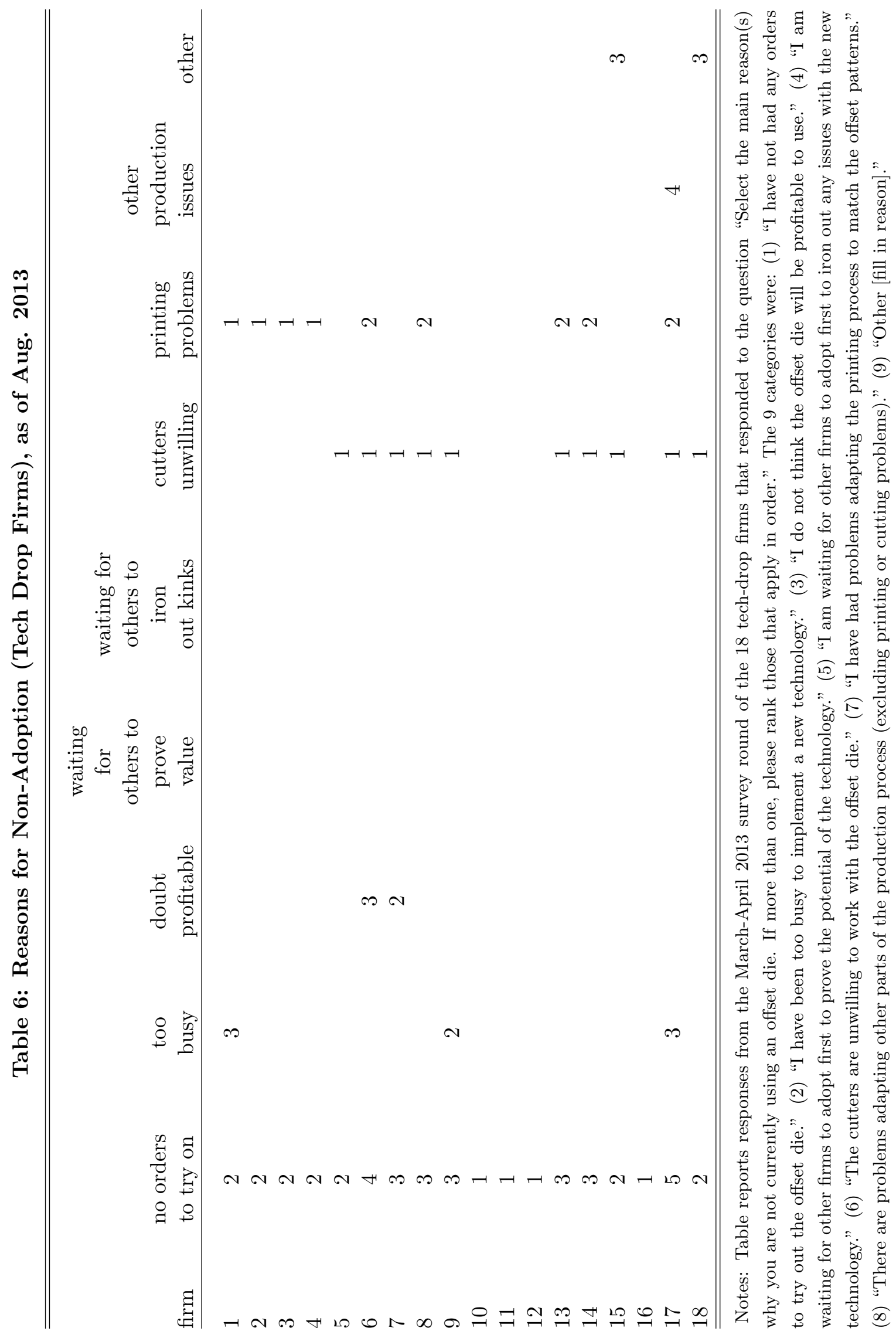


Table 7: Covariate Balance, Incentive-Payment Experiment

\begin{tabular}{|c|c|c|c|}
\hline & $\begin{array}{c}\text { Group A } \\
\text { Incentive Contract } \\
(1) \\
\end{array}$ & $\begin{array}{c}\text { Group B } \\
\text { No Incentive Contract } \\
(2)\end{array}$ & $\begin{array}{c}\text { Difference } \\
(3)\end{array}$ \\
\hline log avg output/month & $\begin{array}{c}9.86 \\
(0.41)\end{array}$ & $\begin{array}{c}9.31 \\
(0.29)\end{array}$ & $\begin{array}{c}0.55 \\
(0.50)\end{array}$ \\
\hline log avg employment & $\begin{array}{c}3.35 \\
(0.38)\end{array}$ & $\begin{array}{c}3.23 \\
(0.25)\end{array}$ & $\begin{array}{c}0.13 \\
(0.45)\end{array}$ \\
\hline log avg price, size 5 promo & $\begin{array}{c}5.40 \\
(0.02)\end{array}$ & $\begin{array}{c}5.45 \\
(0.07)\end{array}$ & $\begin{array}{l}-0.05 \\
(0.07)\end{array}$ \\
\hline log avg price, size 5 training & $\begin{array}{c}6.00 \\
(0.06)\end{array}$ & $\begin{array}{c}5.93 \\
(0.06)\end{array}$ & $\begin{array}{c}0.07 \\
(0.08)\end{array}$ \\
\hline $\operatorname{avg} \%$ promotional (of size 5 ) & $\begin{array}{l}34.90 \\
(6.20)\end{array}$ & $\begin{array}{c}32.04 \\
(7.26)\end{array}$ & $\begin{array}{c}2.86 \\
(9.60)\end{array}$ \\
\hline avg Rs/ball, head cutter & $\begin{array}{c}1.45 \\
(0.10)\end{array}$ & $\begin{array}{c}1.63 \\
(0.15)\end{array}$ & $\begin{array}{c}-0.17 \\
(0.18)\end{array}$ \\
\hline CEO university indicator & $\begin{array}{c}0.56 \\
(0.18)\end{array}$ & $\begin{array}{c}0.36 \\
(0.15)\end{array}$ & $\begin{array}{c}0.19 \\
(0.23)\end{array}$ \\
\hline CEO experience & $\begin{array}{l}15.50 \\
(3.60)\end{array}$ & $\begin{array}{l}16.50 \\
(3.60)\end{array}$ & $\begin{array}{l}-1.00 \\
(5.13)\end{array}$ \\
\hline age of firm & $\begin{array}{l}24.53 \\
(2.83)\end{array}$ & $\begin{array}{l}20.60 \\
(2.28)\end{array}$ & $\begin{array}{c}3.93 \\
(3.64)\end{array}$ \\
\hline $\mathrm{N}$ & 15 & 16 & 31 \\
\hline
\end{tabular}

Notes: Table reports baseline balance in the Incentive-Payment Experiment (Experiment 2). Sample is the 31 techdrop firms from the Tech-Drop Experiment who were still active as of September 2013. There are no significant differences between treatment and control groups. Standard errors in parentheses. 
Table 8: Incentive-Payment Experiment (Liberal Adoption Measure)

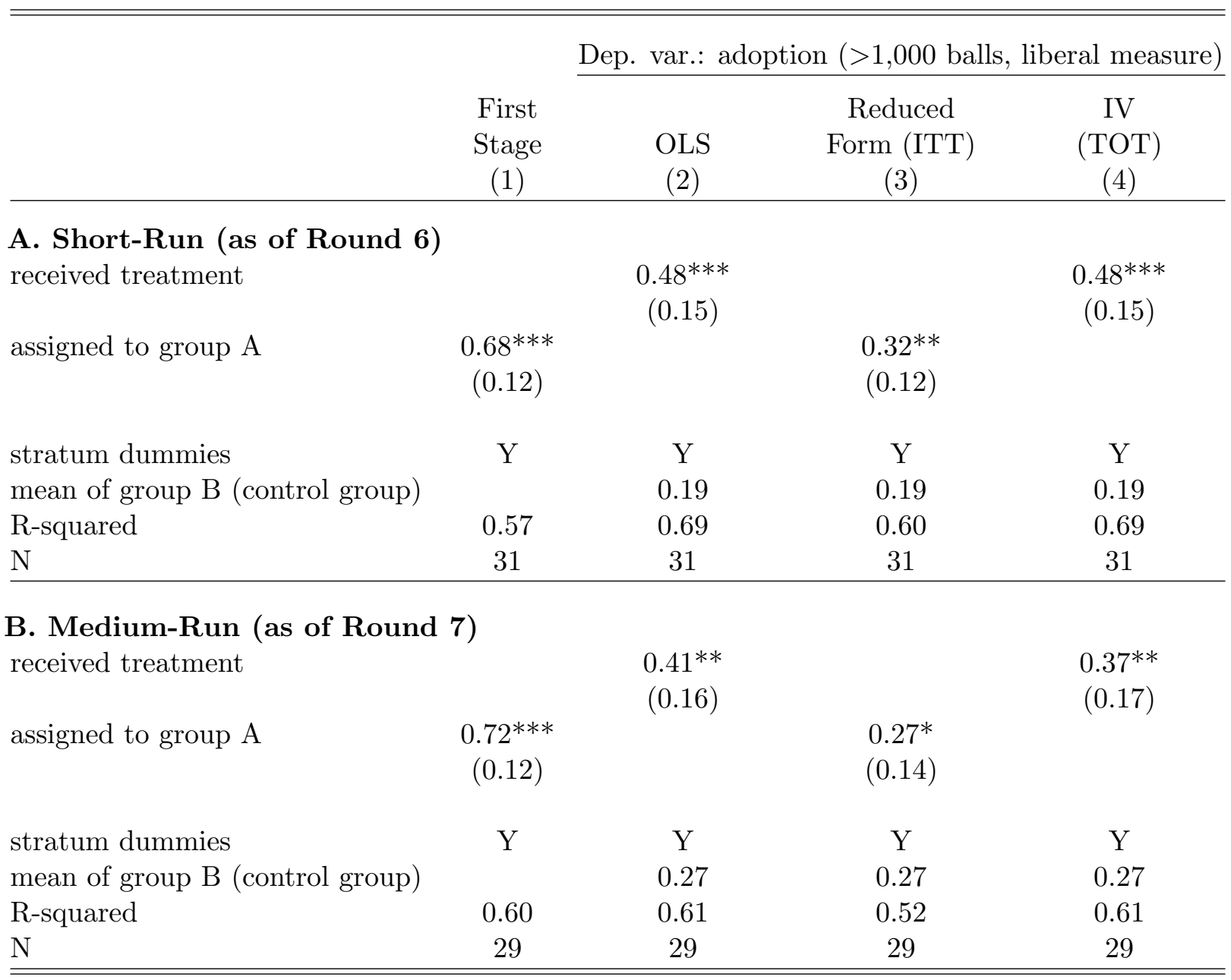

Notes: Table reports results of incentive-payment experiment on adoption rates using the liberal definition of adoption. Panel A reports short-run results as of Round 6 (Jan.-March 2014). Panel B reports medium-run results as of Round 7 (Oct.-Dec. 2014). The dependent variable in Column 1 is an indicator variable for whether the firm received treatment. Two firms exited between Rounds 6 and 7. All regressions include stratum dummies, and report robust standard errors. Significance: * $0.10, * * 0.05 ; * * * 0.01$. 
Table 9: Incentive-Payment Experiment (Conservative Adoption Measure)

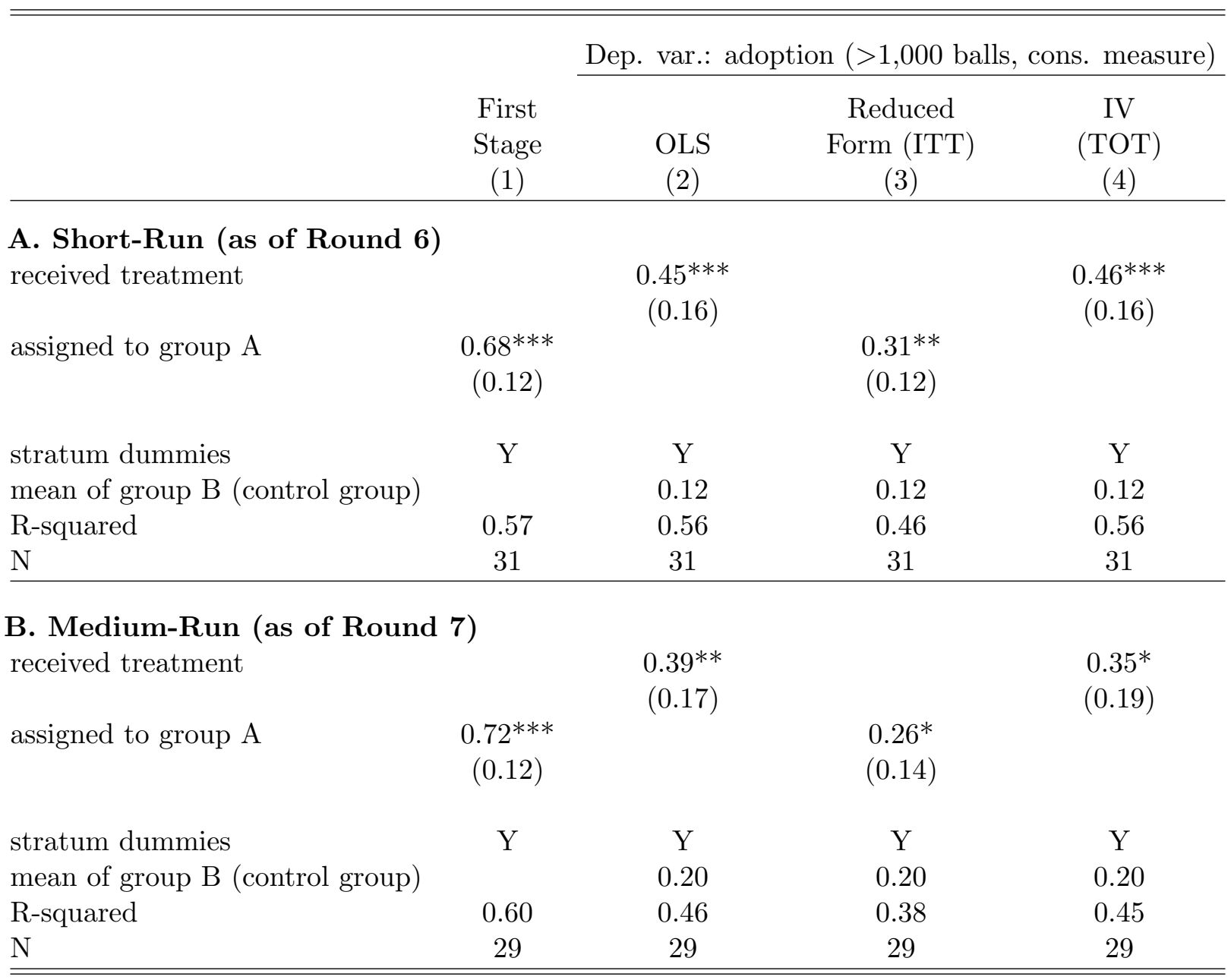

Notes: Table reports results of incentive-payment experiment on adoption rates using the conservative definition of adoption. Panel A reports short-run results as of Round 6 (Jan.-March 2014). Panel B reports medium-run results as of Round 7 (Oct.-Dec. 2014). The dependent variable in Column 1 is an indicator variable for whether the firm received treatment. Two firms exited between Rounds 6 and 7. All regressions include stratum dummies, and report robust standard errors. Significance: * $0.10, * * 0.05 ; * * 0.01$. 
Table 10: Incentive-Payment Experiment Results (Die Purchase as Outcome)

\begin{tabular}{|c|c|c|c|c|}
\hline & \multirow[b]{2}{*}{$\begin{array}{l}\text { First } \\
\text { Stage } \\
(1) \\
\end{array}$} & \multicolumn{3}{|c|}{ Dep. var.: purchased offset die after Sept. 1, 2013} \\
\hline & & $\begin{array}{c}\text { OLS } \\
(2) \\
\end{array}$ & $\begin{array}{c}\text { Reduced } \\
\text { Form (ITT) } \\
(3)\end{array}$ & $\begin{array}{c}\text { IV } \\
(\mathrm{TOT}) \\
(4)\end{array}$ \\
\hline \multicolumn{5}{|c|}{ A. Short-Run (as of Round 6) } \\
\hline \multicolumn{2}{|c|}{ received treatment } & $\begin{array}{c}0.42^{* *} \\
(0.15)\end{array}$ & & $\begin{array}{c}0.40^{* *} \\
(0.16)\end{array}$ \\
\hline assigned to group A & $\begin{array}{c}0.68 * * * \\
(0.12)\end{array}$ & & $\begin{array}{c}0.27^{* *} \\
(0.12)\end{array}$ & \\
\hline stratum dummies & $\mathrm{Y}$ & $\mathrm{Y}$ & $\mathrm{Y}$ & $\mathrm{Y}$ \\
\hline mean of group B (control) & & 0.00 & 0.00 & 0.00 \\
\hline R-squared & 0.57 & 0.40 & 0.24 & 0.40 \\
\hline $\mathrm{N}$ & 31 & 31 & 31 & 31 \\
\hline \multicolumn{5}{|c|}{ B. Medium-Run (as of Round 7) } \\
\hline received treatment & & $\begin{array}{c}0.41^{* *} \\
(0.15)\end{array}$ & & $\begin{array}{c}0.38^{* *} \\
(0.16)\end{array}$ \\
\hline assigned to group A & $\begin{array}{c}0.72^{* * *} \\
(0.12)\end{array}$ & & $\begin{array}{c}0.28^{* *} \\
(0.12)\end{array}$ & \\
\hline stratum dummies & $\mathrm{Y}$ & $\mathrm{Y}$ & $\mathrm{Y}$ & $\mathrm{Y}$ \\
\hline mean of group B (control) & & 0.00 & 0.00 & 0.00 \\
\hline R-squared & 0.60 & 0.40 & 0.24 & 0.40 \\
\hline $\mathrm{N}$ & 29 & 29 & 29 & 29 \\
\hline
\end{tabular}

Notes: Table reports results of incentive-payment experiment on adoption rates using additional die purchases (beyond the trade-in offer) after September 1, 2013 as the measure of adoption. Panel A reports short-run results as of Round 6 (Jan.-March 2014). Panel B reports medium-run results as of Round 7 (Oct.-Dec. 2014). The dependent variable in Column 1 is an indicator variable for whether the firm received treatment. Two firms exited between Rounds 6 and 7 . All regressions include stratum dummies, and report robust standard errors. Significance: * 0.10 , ** 0.05 ; *** 0.01 . 
Table 11: Quantitative Plausibility of Learning-Subsidy Explanation

\begin{tabular}{|c|c|c|c|c|c|c|}
\hline & \multicolumn{6}{|c|}{ Value of prior for whether offset die works as described } \\
\hline & $\begin{array}{l}.01 \\
(1) \\
\end{array}$ & $\begin{array}{l}.05 \\
(2) \\
\end{array}$ & $\begin{array}{l}.1 \\
(3) \\
\end{array}$ & $\begin{array}{l}.25 \\
(4) \\
\end{array}$ & $\begin{array}{c}.5 \\
(5) \\
\end{array}$ & $\begin{array}{c}1 \\
(6) \\
\end{array}$ \\
\hline \multicolumn{7}{|c|}{ A. Estimates of fixed costs } \\
\hline estimate of $\mu$ & $\begin{array}{c}6.46^{* * *} \\
(0.50)\end{array}$ & $\begin{array}{c}7.41^{* * *} \\
(0.30)\end{array}$ & $\begin{array}{c}8.00 * * * \\
(0.29)\end{array}$ & $\begin{array}{c}8.85^{* * *} \\
(0.28)\end{array}$ & $\begin{array}{c}9.53^{* * *} \\
(0.28)\end{array}$ & $\begin{array}{c}10.21^{* * *} \\
(0.28)\end{array}$ \\
\hline estimate of $\sigma_{\varepsilon}$ & $\begin{array}{l}1.87^{* *} \\
(0.75)\end{array}$ & $\begin{array}{l}1.29^{* *} \\
(0.48)\end{array}$ & $\begin{array}{c}1.23^{* * *} \\
(0.44)\end{array}$ & $\begin{array}{c}1.21^{* * *} \\
(0.41)\end{array}$ & $\begin{array}{c}1.20^{* * *} \\
(0.40)\end{array}$ & $\begin{array}{c}1.20^{* * *} \\
(0.40)\end{array}$ \\
\hline $\mathrm{N}$ & 31 & 31 & 31 & 31 & 31 & 31 \\
\hline $\begin{array}{l}\text { B. ITT estimate } \\
\text { assigned to group A }\end{array}$ & $\begin{array}{c}0.24 \\
(0.11)\end{array}$ & $\begin{array}{c}0.10 \\
(0.09)\end{array}$ & $\begin{array}{c}0.06 \\
(0.06)\end{array}$ & $\begin{array}{c}0.03 \\
(0.04)\end{array}$ & $\begin{array}{c}0.01 \\
(0.03)\end{array}$ & $\begin{array}{c}0.01 \\
(0.02)\end{array}$ \\
\hline $\mathrm{N}$ & 31 & 31 & 31 & 31 & 31 & 31 \\
\hline \multicolumn{7}{|c|}{ C. P-values of observing $\geq 5$ adopters in incentive-payment experiment } \\
\hline & $0.23 \overline{4}$ & 0.013 & 0.000 & 0.000 & 0.000 & 0.000 \\
\hline
\end{tabular}

Notes: Sample is tech-drop firms still active at the time of second experiment (Sept. 2013). Panel A reports estimates for $\mu$ and $\sigma_{\varepsilon}$ from maximizing the likelihood function in equation (3) for the value of prior noted in the column heading. Panel B reports the average and standard deviation of the ITT estimates across 1000 simulation draws of log fixed costs from a normal distribution with mean $\hat{\mu}$ and standard deviation $\hat{\sigma}_{\varepsilon}$ for the corresponding prior. Panel $\mathrm{C}$ reports the p-values of observing 5 or more adopters in the short-run (recall that we observed 5 adopters in the short-run according to the liberal-definition adoption variable), based on the frequency of occurrence in the 1000 simulations, under the null hypothesis that second experiment only changed fixed costs. Significance: $* 0.10, * * 0.05, * * * 0.01$. 


\section{Organizational Barriers to Technology Adoption: Evidence from Soccer-Ball Producers in Pakistan}

David Atkin, Azam Chaudhry, Shamyla Chaudry Amit K. Khandelwal and Eric Verhoogen

July 2016

Appendix A: Appendix Tables and Figures 


\section{Figure A.1: "Buckyball" Design}

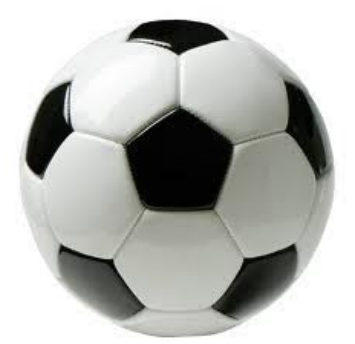

Notes: Figure shows the standard "buckyball" design, based on a geodesic dome designed by R. Buckminster Fuller. It combines 20 hexagons and 12 pentagons.

Figure A.2: U.S. Imports of Inflatable Soccer Balls

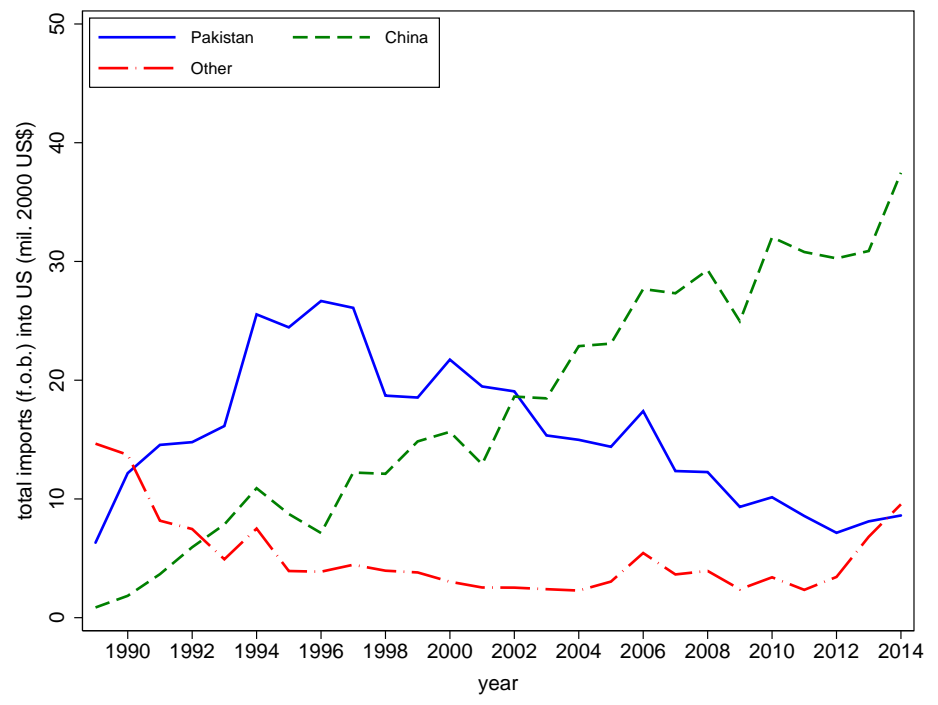

Notes: Figure shows import market shares within the United States in HS 10-digit category 9506.62.40.80 ("inflatable soccer balls"). Primary countries in "other" category are South Korea in early 1990s and Vietnam and Indonesia in 2012-2014. Source: United States International Trade Commission. 
Figure A.3: Making the Laminated Rexine Sheet (Step 1)

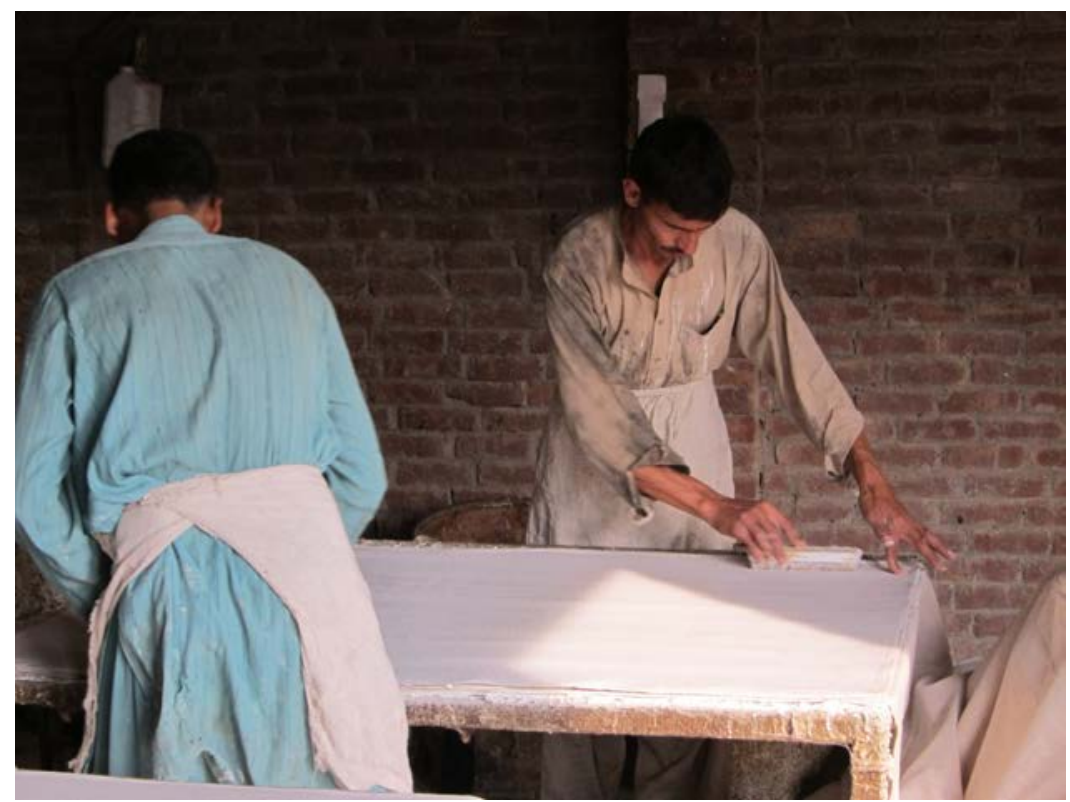

Notes: Figure displays workers gluing layers of cloth (cotton and/or polyester) to artificial leather called rexine using a latex-based adhesive to form what is called a laminated rexine sheet.

Figure A.4: Cutting the Laminated Rexine Sheet (Step 2)

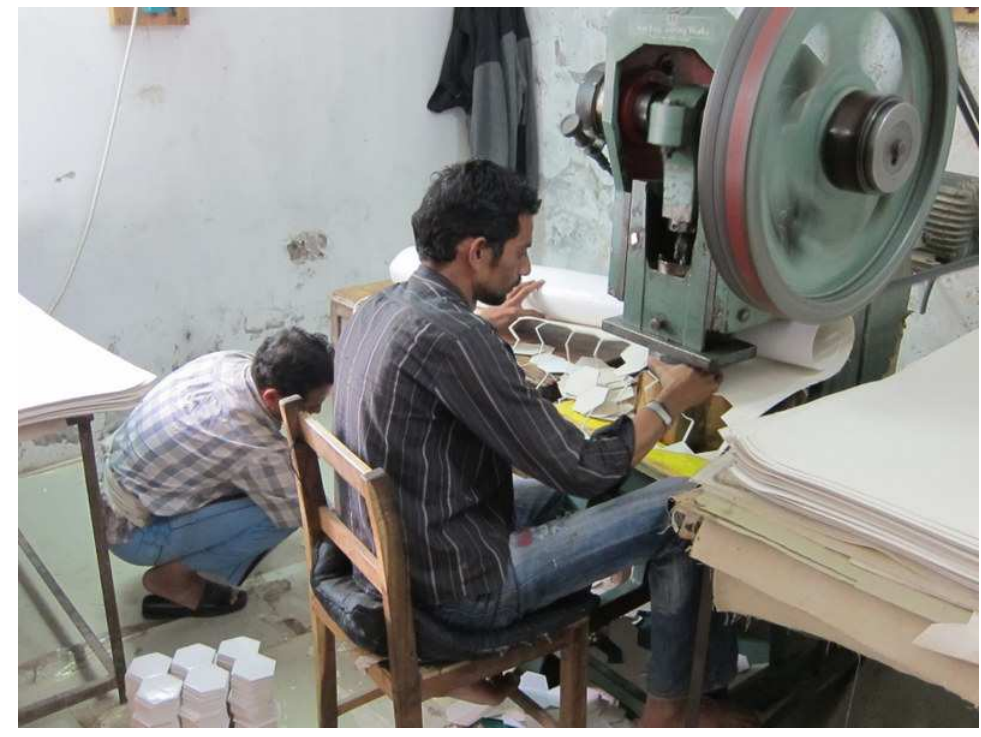

Notes: Figure displays a cutter using a hydraulic press to cut hexagons from the laminated rexine sheet. The process for cutting pentagons differs only in the die used. 
Figure A.5: Printing the Designs (Step 3)

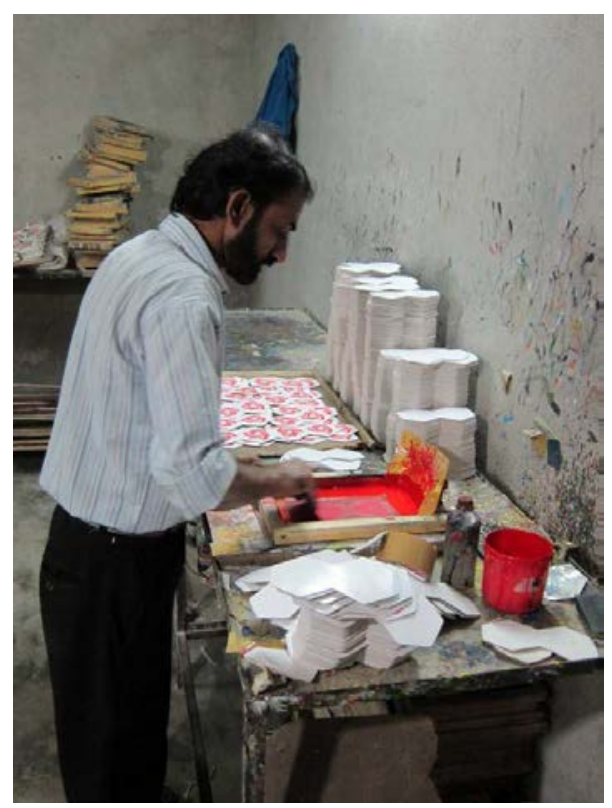

Notes: Figure displays a printer printing a logo on the pentagon and hexagon panels.

Figure A.6: Stitching (Step 4)

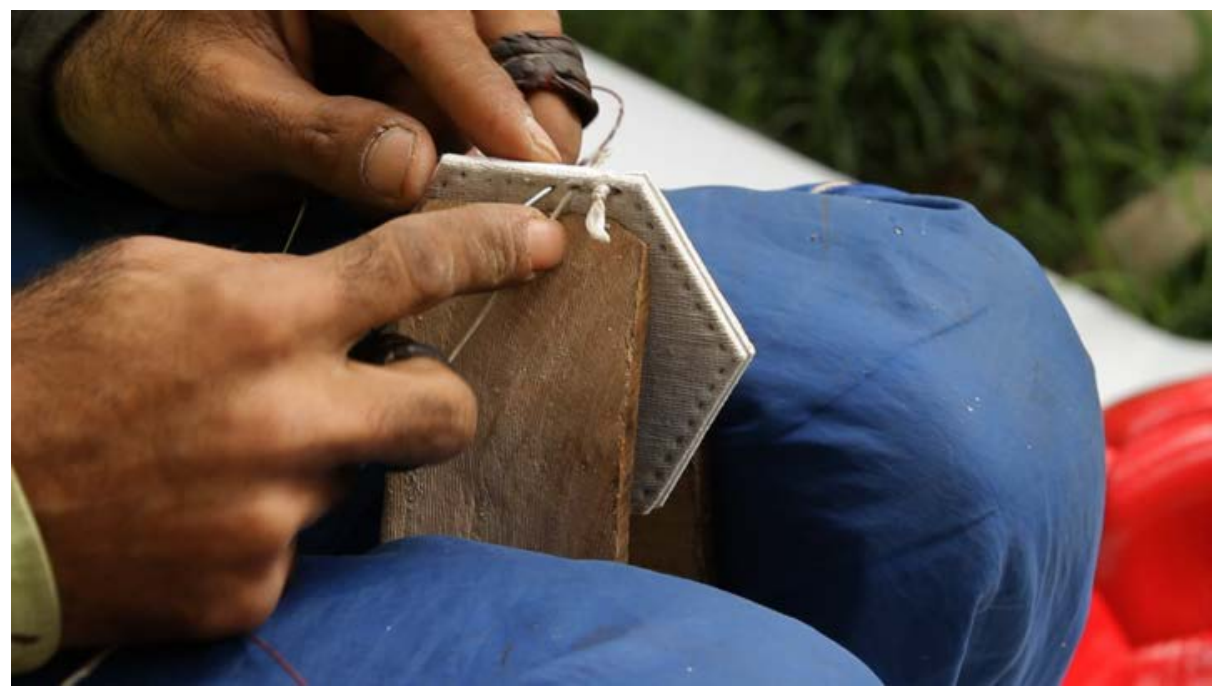

Notes: Figure displays a worker stitching a soccer ball. 
Figure A.7: Snapshot from YouTube Video of Adidas Jabulani Production Process

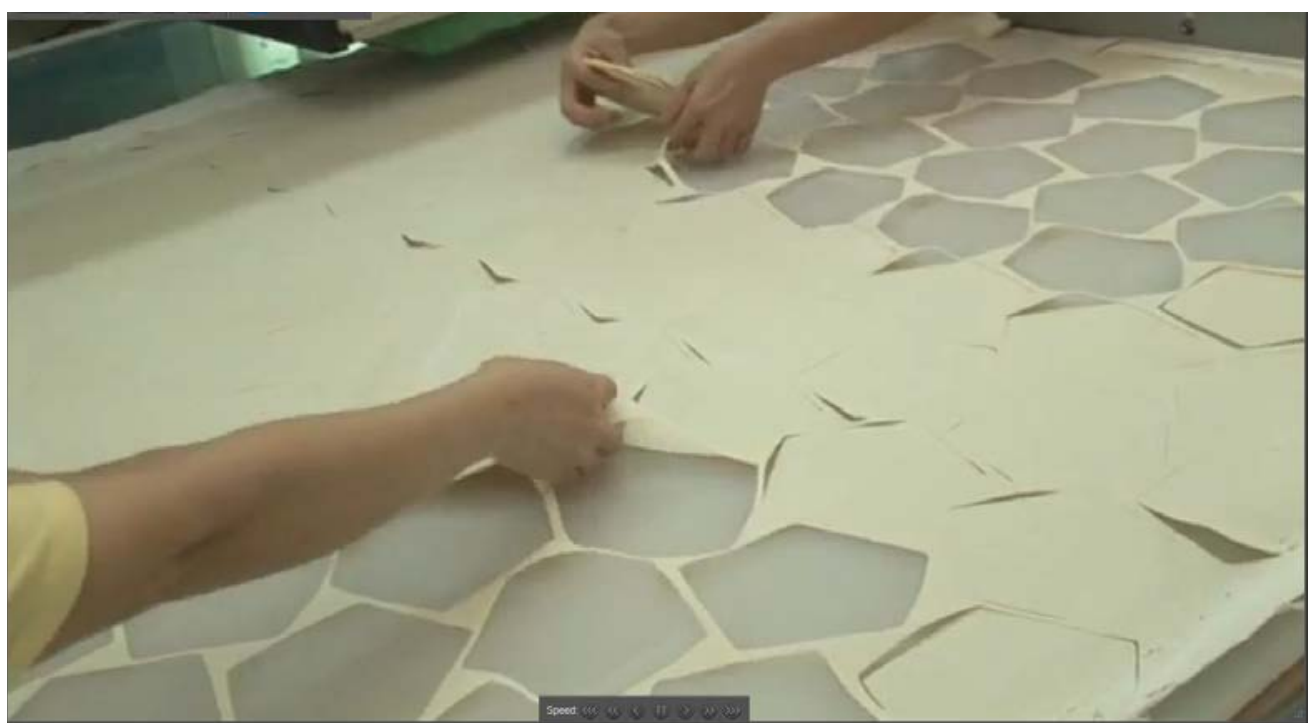

Notes: Snapshot from YouTube video of production process for Adidas Jabulani ball, used in 2010 World Cup, available at http://www.youtube.com/watch?v=zbLjk4OTRdI. Pentagons used for interior lining of ball. Accessed June 10, 2011.

Figure A.8: The "Offset" Four-Pentagon Die

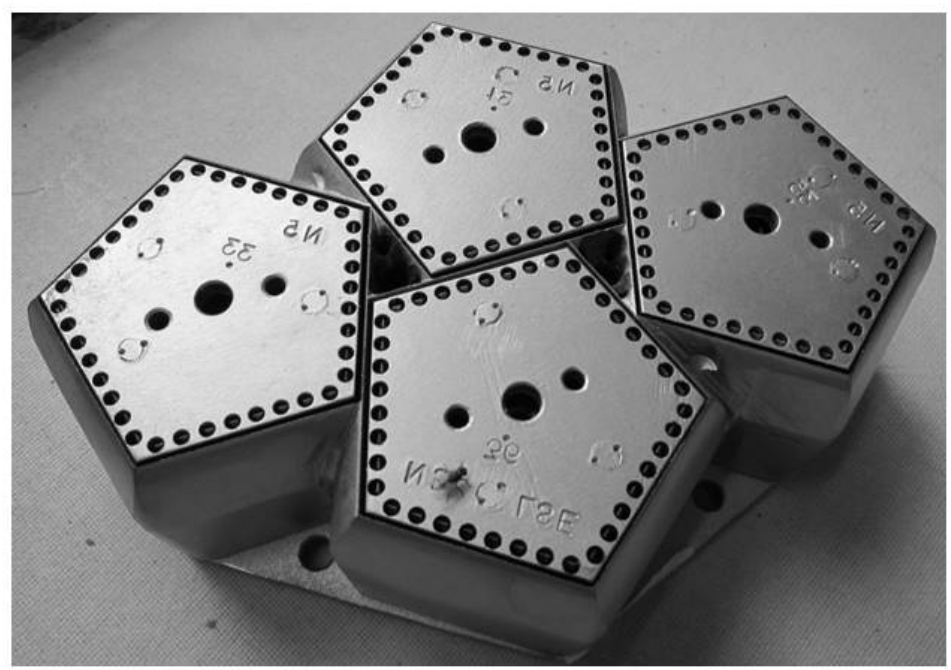

Notes: Figure displays the four-panel offset die that was provided to Tech-Drop firms. 


\section{Figure A.9: Wikipedia "Pentagon" Page}

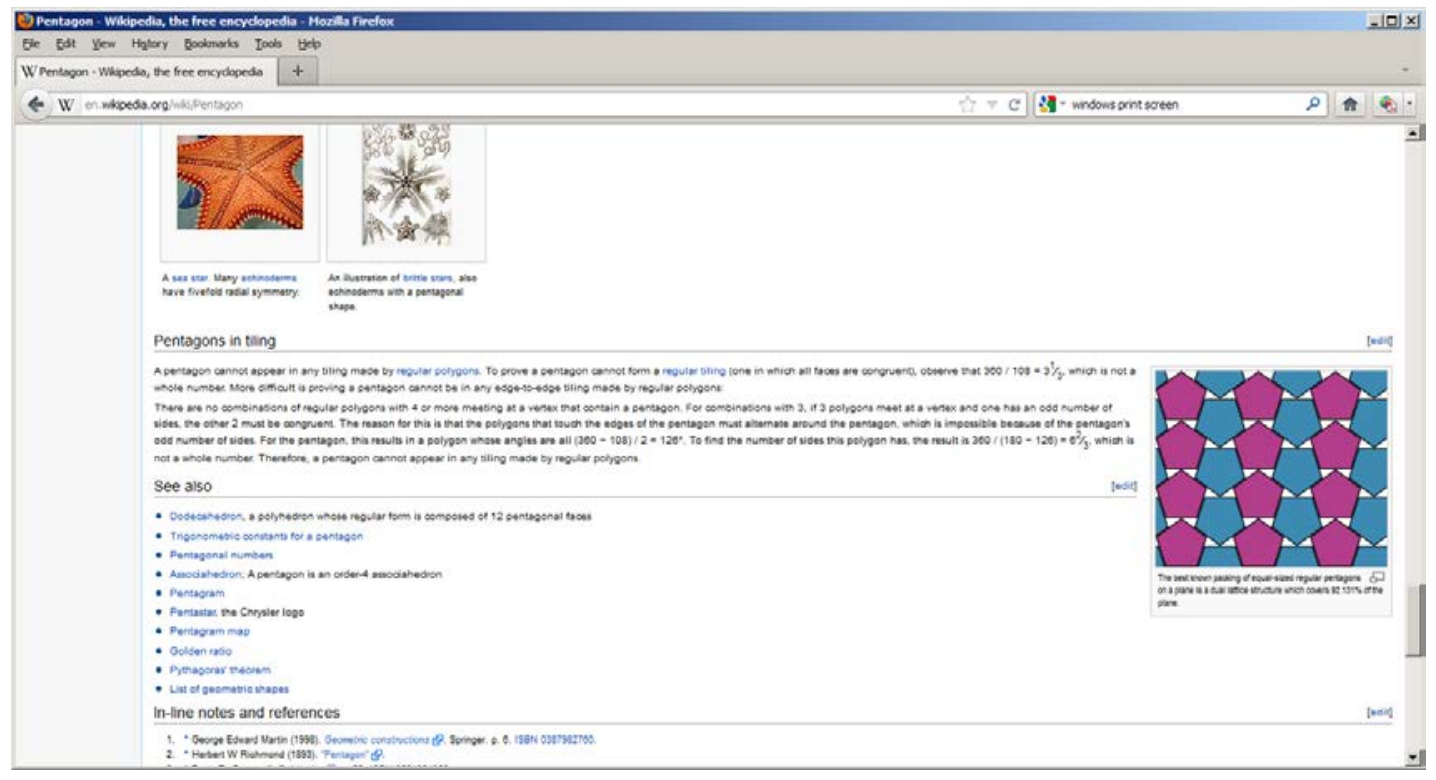

Note: Accessed April 29, 2012.

Figure A.10: Adoption of Offset Dies by Firm Z

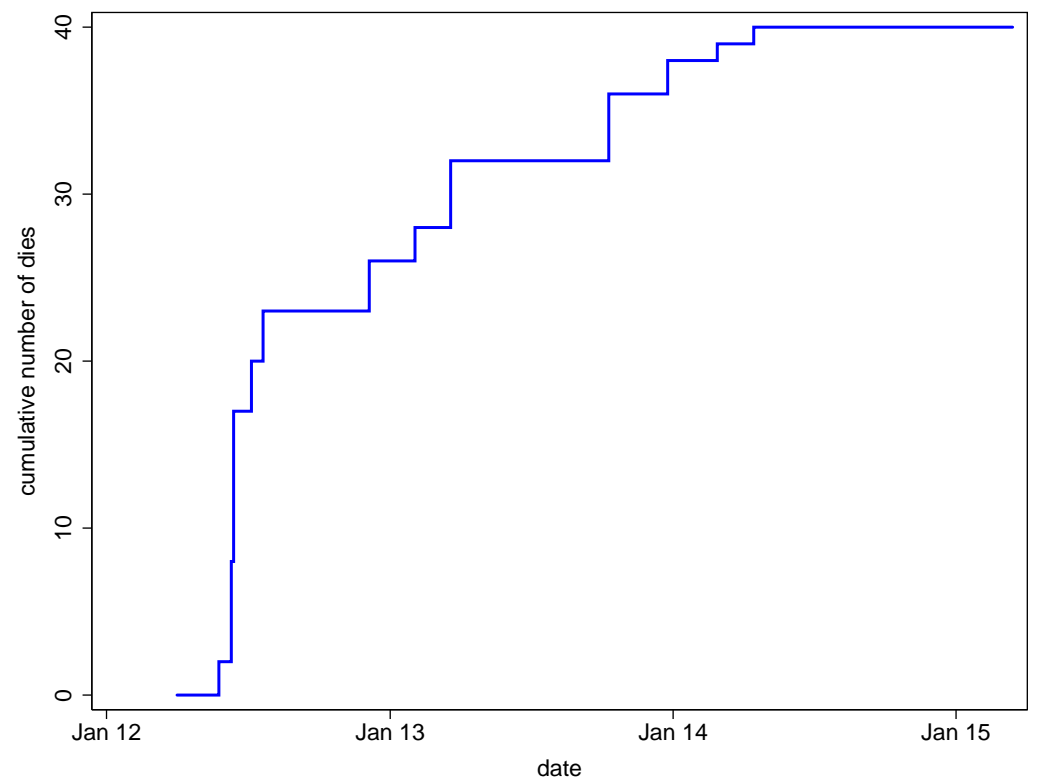

Notes: Figure displays cumulative number of purchases of offset dies by "Firm Z", a large producer which was a late responder assigned to the no-drop group, but which found out almost immediately about the offset die after the initial roll-out in May 2012. By March 2014 the firm reported using offset dies for 100 percent of its pentagon cutting. 


\section{Figure A.11: Permutation Test: Liberal Adoption Measure}

Round 6 (Short Run)

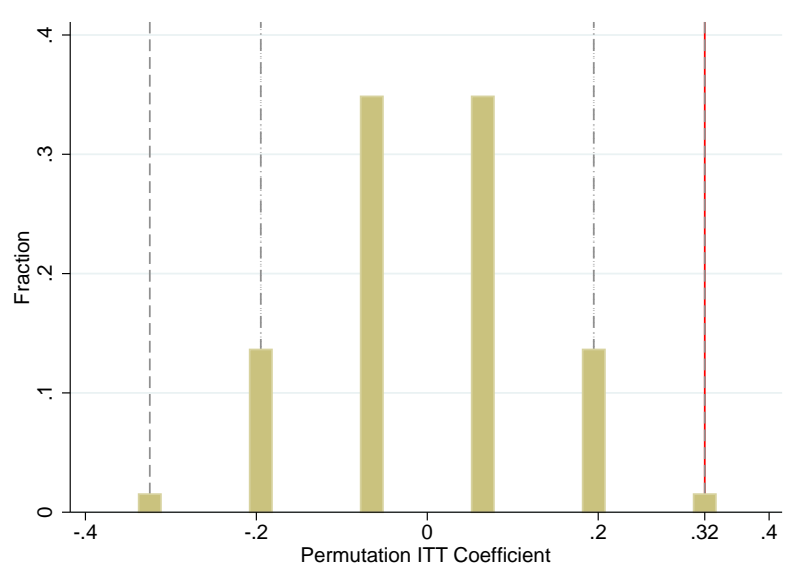

Round 7 (Medium Run)

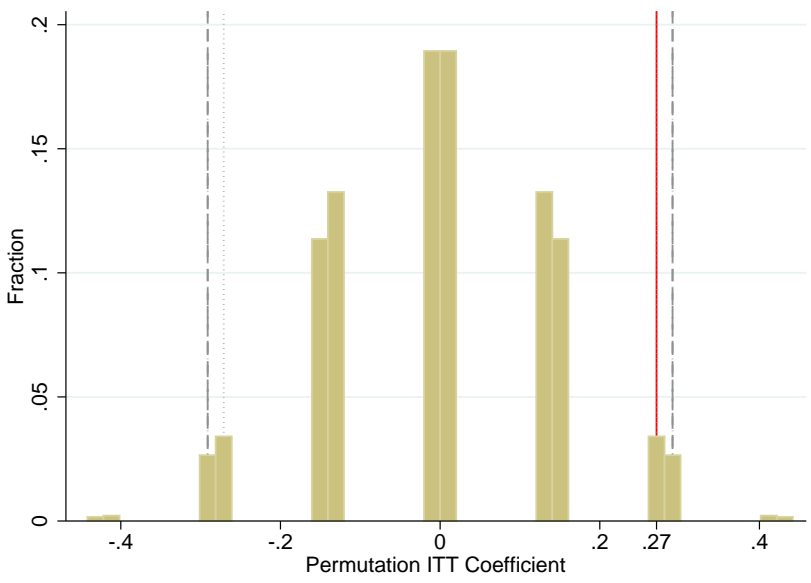

Notes: Figure displays the distribution of ITT coefficients from short-run (left panel) and medium-run (right panel) permutation tests using the liberal adoption measure ( $>1000$ balls cut with offset die, using non-survey as well as survey information). The dotted, dashed-dotted and dashed grey lines reflect critical values for a two-sided hypothesis test of the null that that the ITT effect is zero at a 10\%, $5 \%$ and $1 \%$ level of significance, respectively. The solid red line is the observed ITT estimate from Table 8 and is marked on the x-axis to two decimal places. In the left panel, the $10 \%$ and $5 \%$ lines overlap at both tails, and the $1 \%$ line overlaps with the observed ITT estimate at the right tail. In the right panel, the $1 \%$ and $5 \%$ lines overlap, and the observed ITT estimate overlaps with the $10 \%$ line at the right tail.

Figure A.12: Permutation Test: Conservative Adoption Measure Round 6 (Short-Run) Round 7 (Medium-Run)
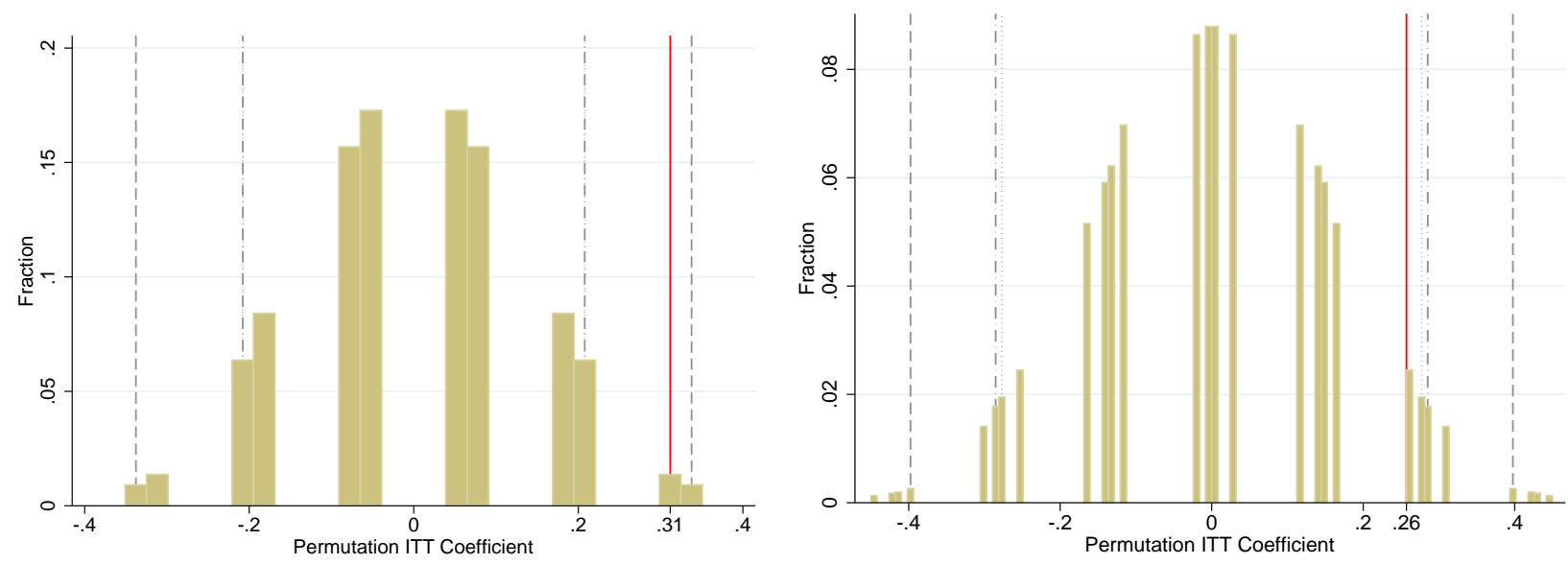

Notes: Figure displays the distribution of ITT coefficients from short-run (left panel) and medium-run (right panel) permutation tests using the conservative adoption measure ( $>1000$ balls cut with offset die, using only survey information). The dotted, dashed-dotted and dashed grey lines reflect critical values for a two-sided hypothesis test that the ITT effect is zero at a $10 \%, 5 \%$ and $1 \%$ level of significance, respectively. The solid red line is the observed ITT estimate from Table 9 and is marked on the $\mathrm{x}$-axis to two decimal places. The $10 \%$ and $5 \%$ lines overlap in the left panel. 
Figure A.13: Permutation Test: Die Purchase

Round 6 (Short-Run)

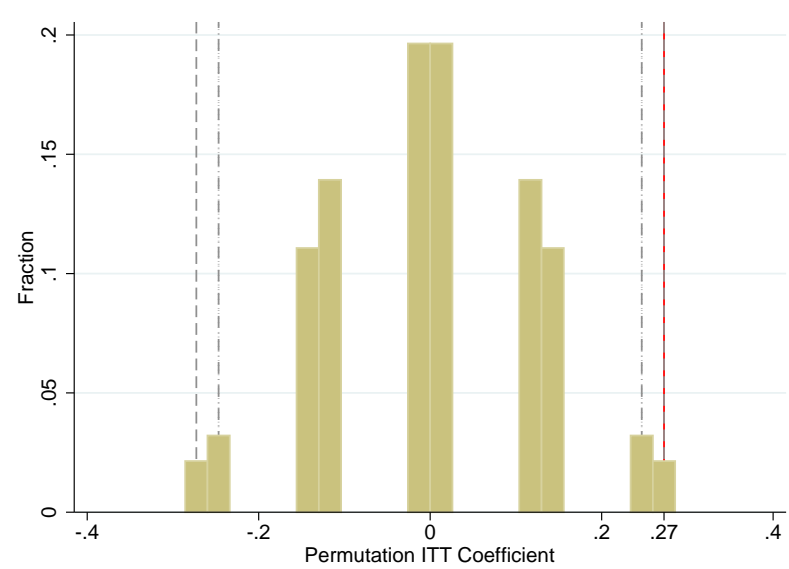

Round 7 (Medium-Run)

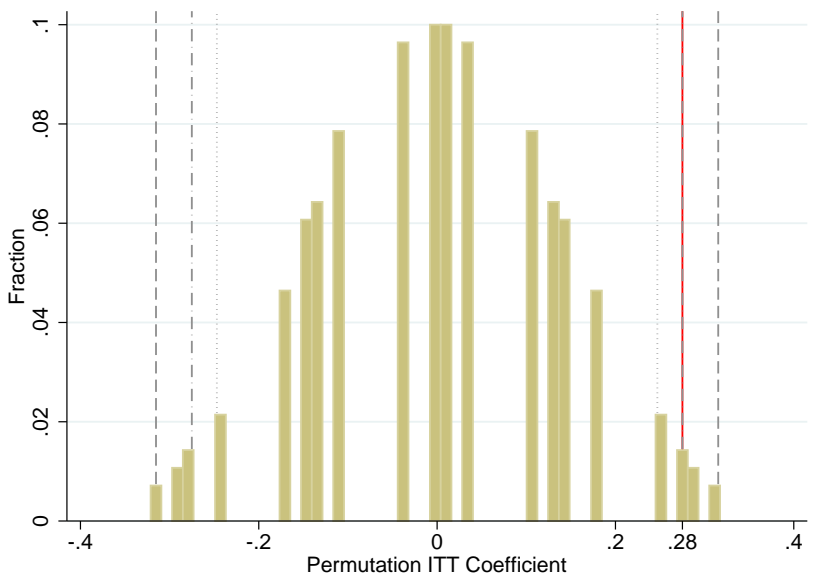

Notes: Figure displays the distribution of ITT coefficients from short-run (left panel) and medium-run (right panel) permutation tests using die purchase after Sept. 2013 as an alternative measure of adoption. The dotted, dasheddotted and dashed grey lines reflect critical values for a two-sided hypothesis test that the ITT effect is zero at a $10 \%, 5 \%$ and $1 \%$ level of significance, respectively. The solid red line is the observed ITT estimate from Table 10 and is marked on the x-axis to two decimal places. In the left panel, the $10 \%$ and $5 \%$ lines overlap at both tails, and the observed ITT estimate overlaps with the $1 \%$ line at the right tail. In the right panel, the $5 \%$ line overlaps with the actual ITT estimate at the right tail.

Figure A.14: Distribution of Piece Rates

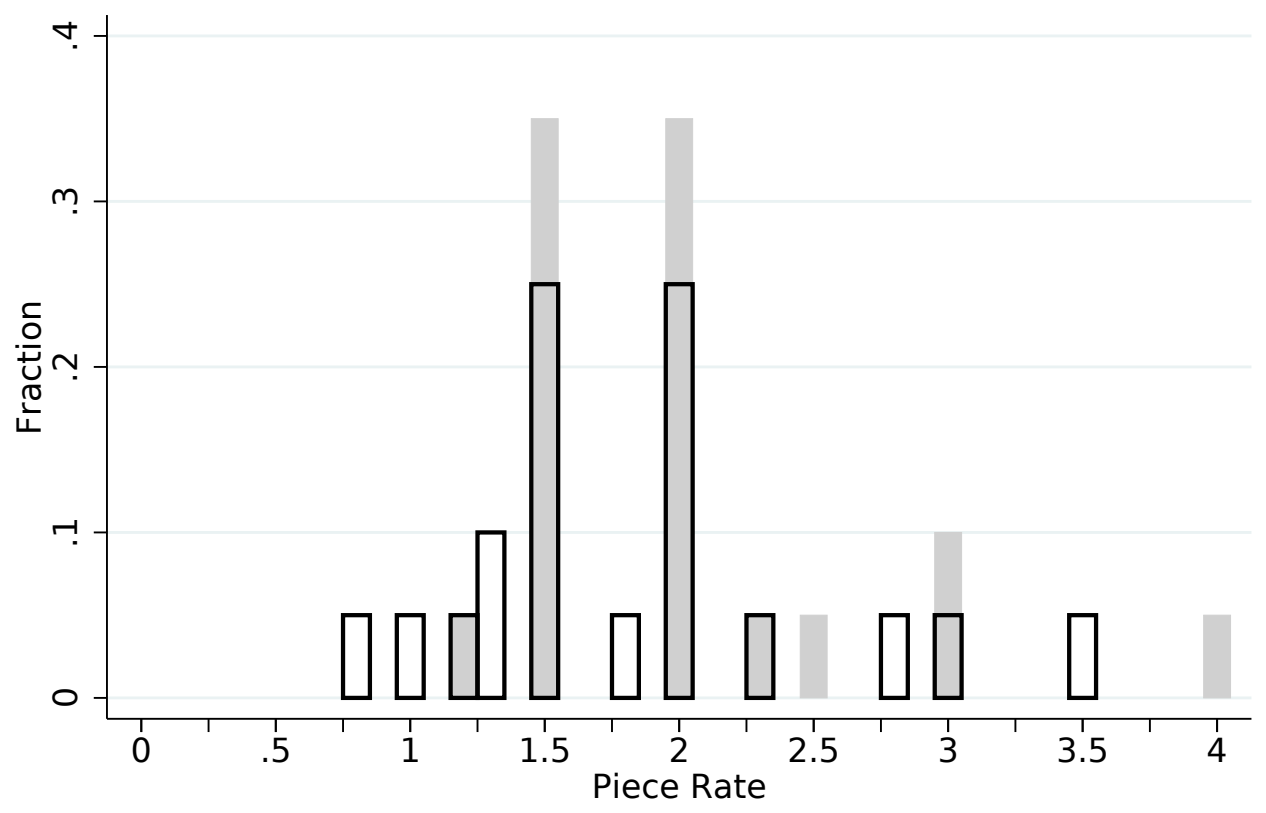

September $2014 \square$ August 2013

Notes: Figure displays the distribution of piece rates paid by firms using data collected in Round 7 of our survey. 
Figure A.15: Effect of Incentive Treatment Under Assumption It Only Reduced Fixed Costs
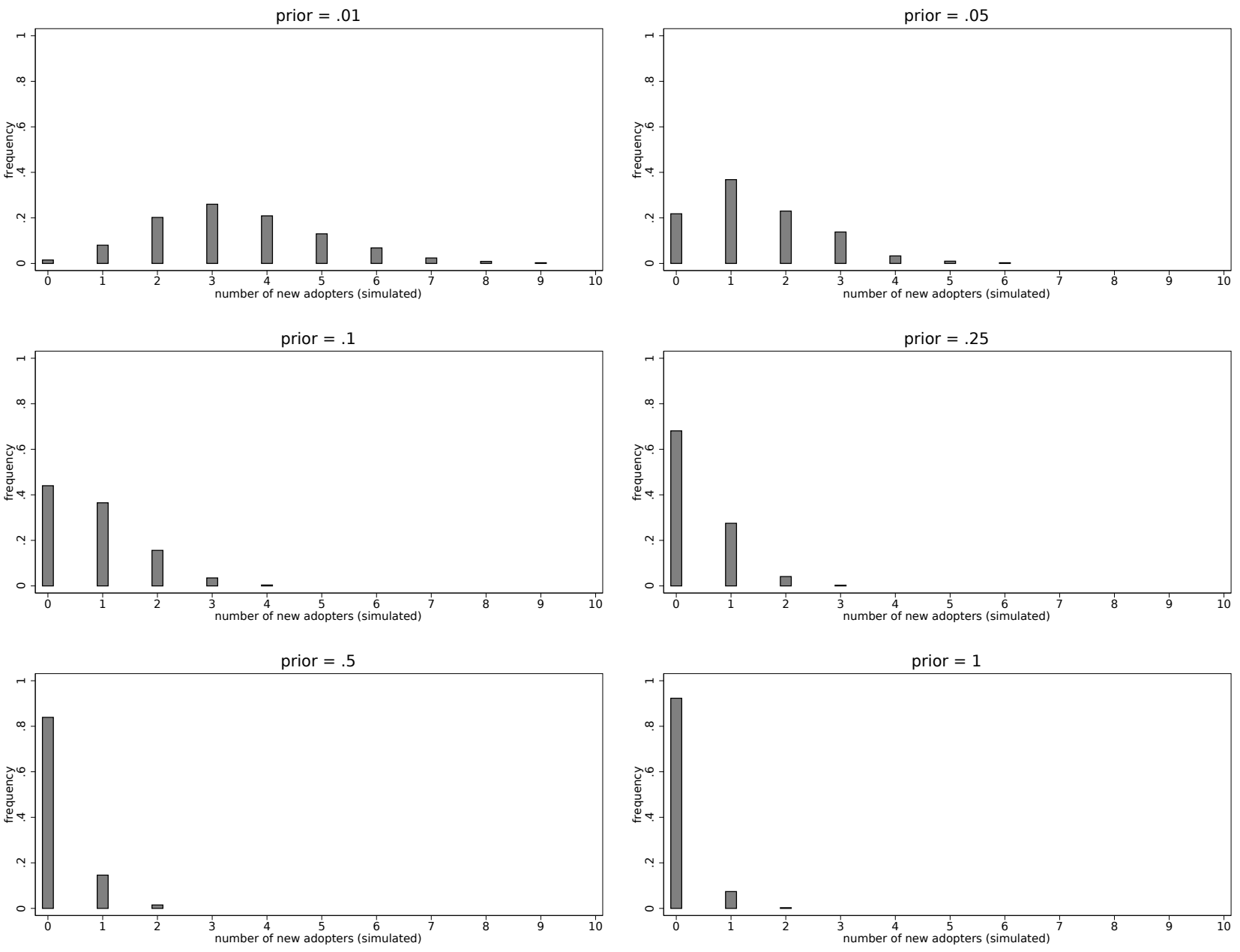

Notes: Figure displays the distribution of the number of firms from Group A predicted to respond to the incentive intervention in the short-run, using 1,000 simulation draws from a normal distribution with mean and standard deviation reported in Table 11, using liberal measure of adoption. See Section 8.1 for more details. 
Table A.1: Production Costs

\begin{tabular}{lcc}
\hline \hline Input & Share of Production Costs $(\%)$ & Input Cost (in Rs) \\
\hline rexine & 19.79 & 39.68 \\
& $(5.37)$ & $(13.87)$ \\
cotton/poly cloth & 12.32 & 23.27 \\
latex & $(4.56)$ & $(8.27)$ \\
& 13.94 & 38.71 \\
bladder & $(10.73)$ & $(90.71)$ \\
labor for cutting & 21.07 & 42.02 \\
& $(4.87)$ & $(14.09)$ \\
labor for stitching & 0.78 & 1.49 \\
& $(0.22)$ & $(0.31)$ \\
other labor & 19.67 & 39.24 \\
& $(5.25)$ & $(12.82)$ \\
overhead & 7.30 & 15.56 \\
& $(4.55)$ & $(13.21)$ \\
total & 5.14 & 10.84 \\
\hline $\mathrm{N}$ & $(2.05)$ & $(6.10)$ \\
\hline \hline
\end{tabular}

Notes: Columns 1 and 2 report the mean cost share per ball of each input and the input cost in Rupees, respectively. "Other labor" includes laminating, washing, packing, and matching. Data taken from the baseline survey. Standard deviations in parentheses. 


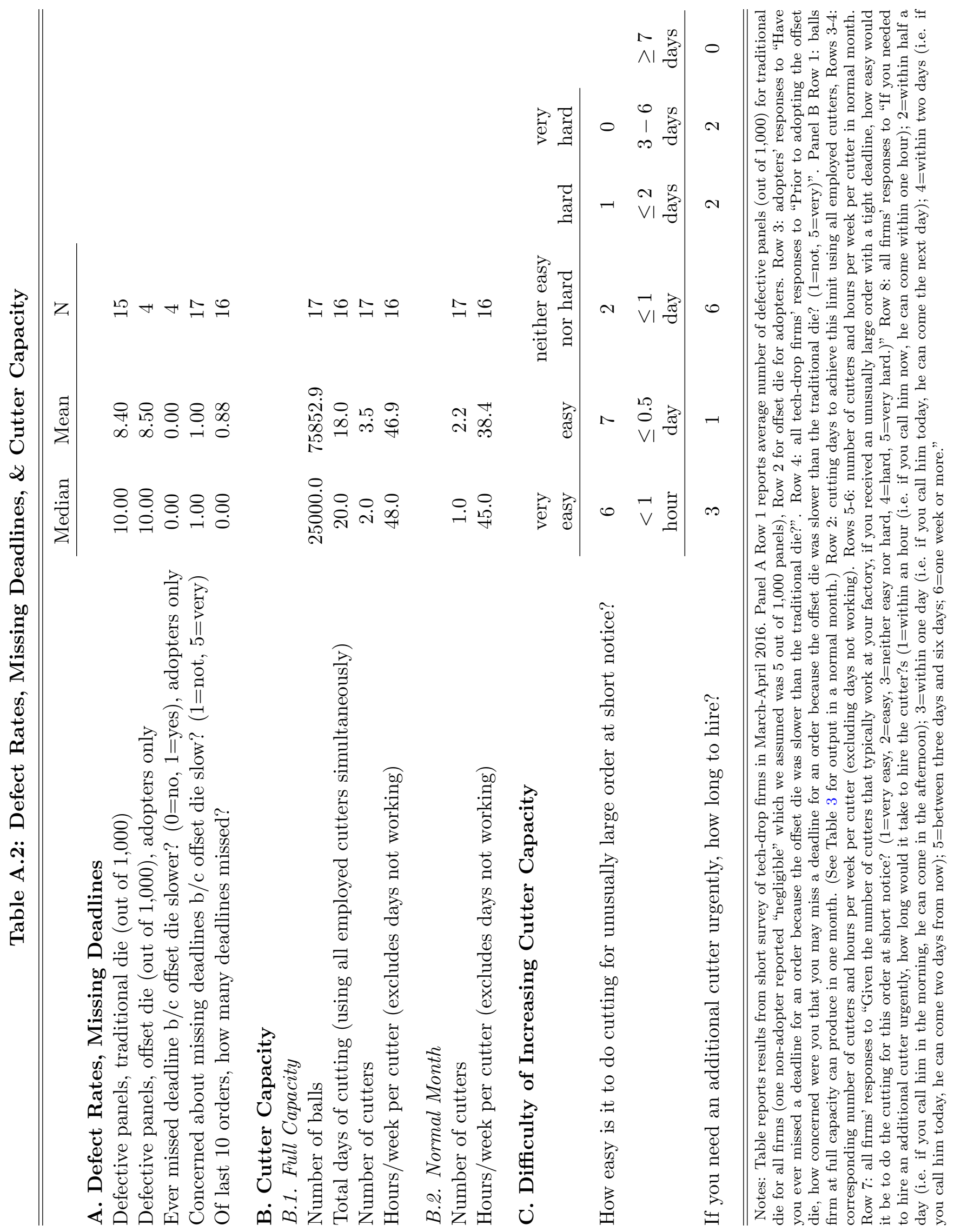


Table A.3: Covariate Balance, Tech-Drop Experiment

\begin{tabular}{|c|c|c|c|}
\hline & Tech Drop & Cash Drop & No Drop \\
\hline \multicolumn{4}{|l|}{ A. Initial responders } \\
\hline output, normal month (000s) & $\begin{array}{c}34.18 \\
(11.48)\end{array}$ & $\begin{array}{c}26.69 \\
(12.15)\end{array}$ & $\begin{array}{l}41.56 \\
(9.53)\end{array}$ \\
\hline output, previous year (000s) & $\begin{array}{c}680.17 \\
(220.13)\end{array}$ & $\begin{array}{c}579.97 \\
(225.13)\end{array}$ & $\begin{array}{c}763.33 \\
(232.95)\end{array}$ \\
\hline employment, normal month & $\begin{array}{c}42.26 \\
(13.25)\end{array}$ & $\begin{array}{c}82.58 \\
(47.16)\end{array}$ & $\begin{array}{c}92.62 \\
(35.77)\end{array}$ \\
\hline$\%$ size 5 & $\begin{array}{l}84.61 \\
(5.38)\end{array}$ & $\begin{array}{l}88.96 \\
(4.52)\end{array}$ & $\begin{array}{l}82.67 \\
(3.74)\end{array}$ \\
\hline$\%$ promotional (of size 5 ) & $\begin{array}{l}50.12 \\
(7.12)\end{array}$ & $\begin{array}{c}66.09 \\
(11.04)\end{array}$ & $\begin{array}{l}59.02 \\
(5.17)\end{array}$ \\
\hline age of firm & $\begin{array}{l}22.70 \\
(2.25)\end{array}$ & $\begin{array}{l}29.25 \\
(4.88)\end{array}$ & $\begin{array}{l}25.76 \\
(3.09)\end{array}$ \\
\hline CEO experience & $\begin{array}{l}16.22 \\
(2.39)\end{array}$ & $\begin{array}{l}20.42 \\
(2.70)\end{array}$ & $\begin{array}{l}16.55 \\
(1.62)\end{array}$ \\
\hline CEO college indicator & $\begin{array}{c}0.43 \\
(0.11)\end{array}$ & $\begin{array}{c}0.27 \\
(0.14)\end{array}$ & $\begin{array}{c}0.40 \\
(0.08)\end{array}$ \\
\hline head cutter experience & $\begin{array}{l}17.00 \\
(2.08)\end{array}$ & $\begin{array}{l}30.33 \\
(6.69)\end{array}$ & $\begin{array}{l}20.91 \\
(2.68)\end{array}$ \\
\hline head cutter tenure & $\begin{array}{l}12.20 \\
(2.21)\end{array}$ & $\begin{array}{l}12.00 \\
(5.77)\end{array}$ & $\begin{array}{l}10.50 \\
(2.11)\end{array}$ \\
\hline share cutters paid piece rate & $\begin{array}{c}1.00 \\
(0.00)\end{array}$ & $\begin{array}{c}0.83 \\
(0.11)\end{array}$ & $\begin{array}{c}0.89 \\
(0.05)\end{array}$ \\
\hline rupees/ball (head cutter) & $\begin{array}{c}1.44 \\
(0.14)\end{array}$ & $\begin{array}{c}1.62 \\
(0.21)\end{array}$ & $\begin{array}{c}1.37 \\
(0.10)\end{array}$ \\
\hline $\mathrm{N}$ & 23 & 12 & 50 \\
\hline \multicolumn{4}{|l|}{ B. Initial non-responders } \\
\hline output, normal month (000s) & $\begin{array}{c}27.85 \\
(14.01)\end{array}$ & $\begin{array}{l}34.80 \\
(4.99)\end{array}$ & $\begin{array}{c}63.12 \\
(18.25)\end{array}$ \\
\hline employment, normal month & $\begin{array}{c}67.20 \\
(48.18)\end{array}$ & $\begin{array}{c}61.00 \\
(34.94)\end{array}$ & $\begin{array}{c}353.38 \\
(264.52)\end{array}$ \\
\hline$\%$ size 5 & $\begin{array}{l}68.00 \\
(9.80)\end{array}$ & $\begin{array}{c}72.22 \\
(16.16)\end{array}$ & $\begin{array}{c}96.88 \\
(3.12)\end{array}$ \\
\hline$\%$ promotional (of size 5 ) & $\begin{array}{l}31.17 \\
(9.77)\end{array}$ & $\begin{array}{c}36.11 \\
(12.58)\end{array}$ & $\begin{array}{c}24.22 \\
(13.28)\end{array}$ \\
\hline age of firm & $\begin{array}{l}17.40 \\
(3.13)\end{array}$ & $\begin{array}{c}39.60 \\
(16.68)\end{array}$ & $\begin{array}{l}35.12 \\
(5.55)\end{array}$ \\
\hline $\mathrm{N}$ & 10 & 5 & 8 \\
\hline
\end{tabular}

Notes: Table reports balance for initial responders (i.e. responders to baseline) (Panel A) and initial nonresponders (Panel B). There are no significant differences between groups at the 95 percent level in the initial responder sampler. The initial non-responder sample has significant differences, consistent with the fact that response rates responded to treatment assignment among initial non-responders. Only 23 initial non-responder firms completed an abridged baseline survey which is why the number of observations in Panel B is lower than that reported in Row 1 of Panel B of Table 4; the remaining 8 firms only completed one or more subsequent surveys. Standard errors in parentheses. 


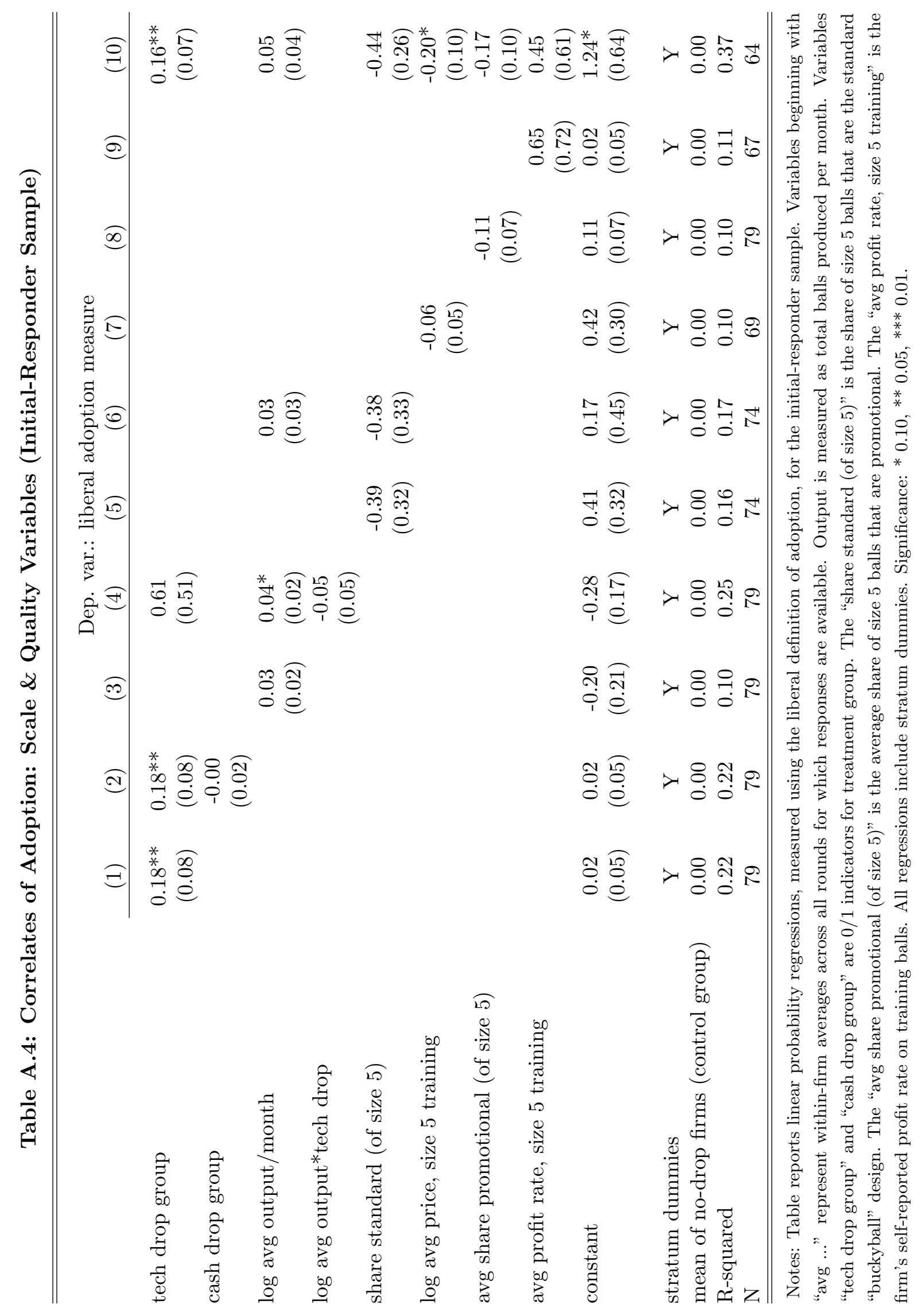




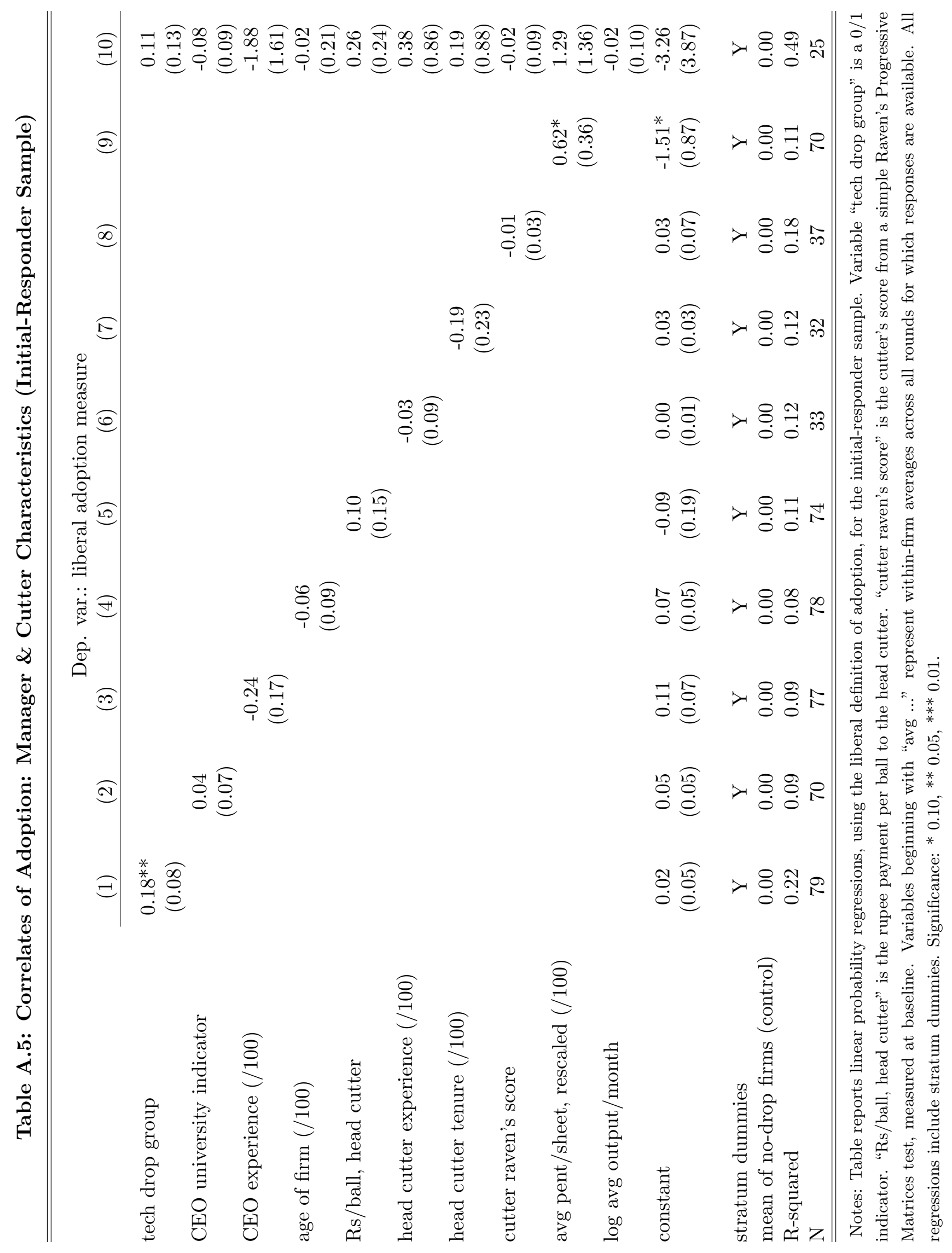


Table A.6: "Test" Results

\begin{tabular}{|c|c|c|c|c|c|c|c|c|c|c|}
\hline firm & 1 & 2 & 3 & 4 & 5 & 6 & 7 & 8 & 9 & 10 \\
\hline time & $2: 52$ & $2: 40$ & $3: 03$ & 3:02 & $2: 59$ & $2: 28$ & $2: 25$ & $2: 45$ & $2: 30$ & $2: 50$ \\
\hline die size & 43.5 & 43.75 & 44 & 44 & 43.5 & 43.5 & 43.5 & 43.5 & 44 & 43.5 \\
\hline \# pentagons & 270 & 272 & 273 & 272 & 282 & 279 & 279 & 272 & 272 & 267 \\
\hline
\end{tabular}

Notes: Table reports the times achieved by cutters at the 10 Group A firms who agreed to the incentive payment intervention. The 2 nd row reports the time, in minutes and seconds, to cut a single laminated rexine sheet with the offset die. The 3rd row reports the size of the die (in $\mathrm{mm}$ ) used by the cutter. The 4 th row reports the number of pentagons achieved. The typical time to cut a sheet with the traditional die is $2: 15$. 
Table A.7: Incentive-Payment Experiment (5,000-ball cutoff)

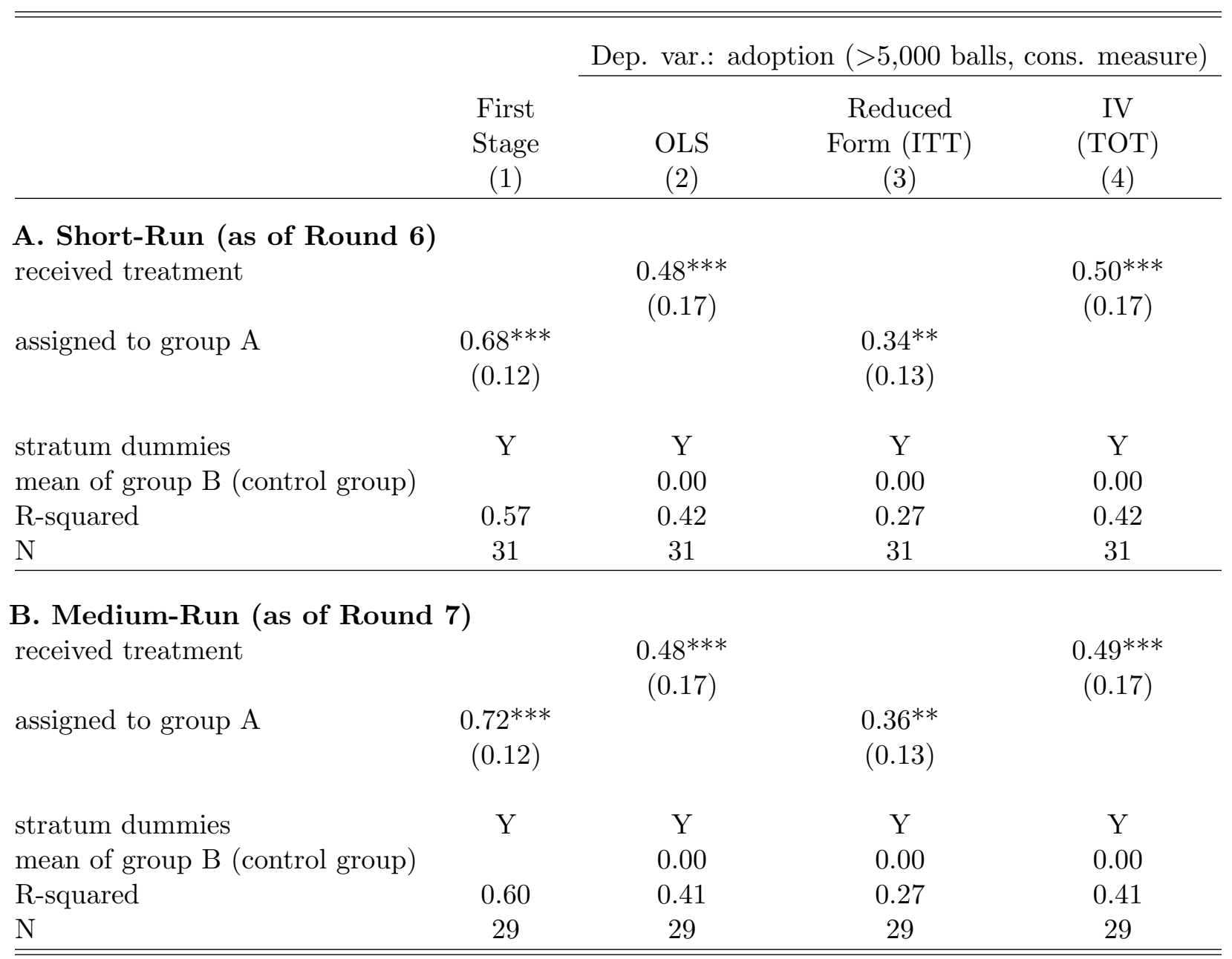

Notes: Table similar to Table 9 in main text but using 5,000-ball cutoff in conservative definition of adoption. Panel A reports short-run results as of Round 6 (Jan.-March 2014). Panel B reports medium-run results as of Round 7 (Oct.-Dec. 2014). The dependent variable in Column 1 is an indicator variable for whether the firm received treatment. Two firms exited between Rounds 6 and 7. All regressions include stratum dummies, and report robust standard errors. Significance: $* 0.10, * * 0.05 ; * * * 0.01$. 
Table A.8: Incentive-Payment Experiment (10,000-ball cutoff)

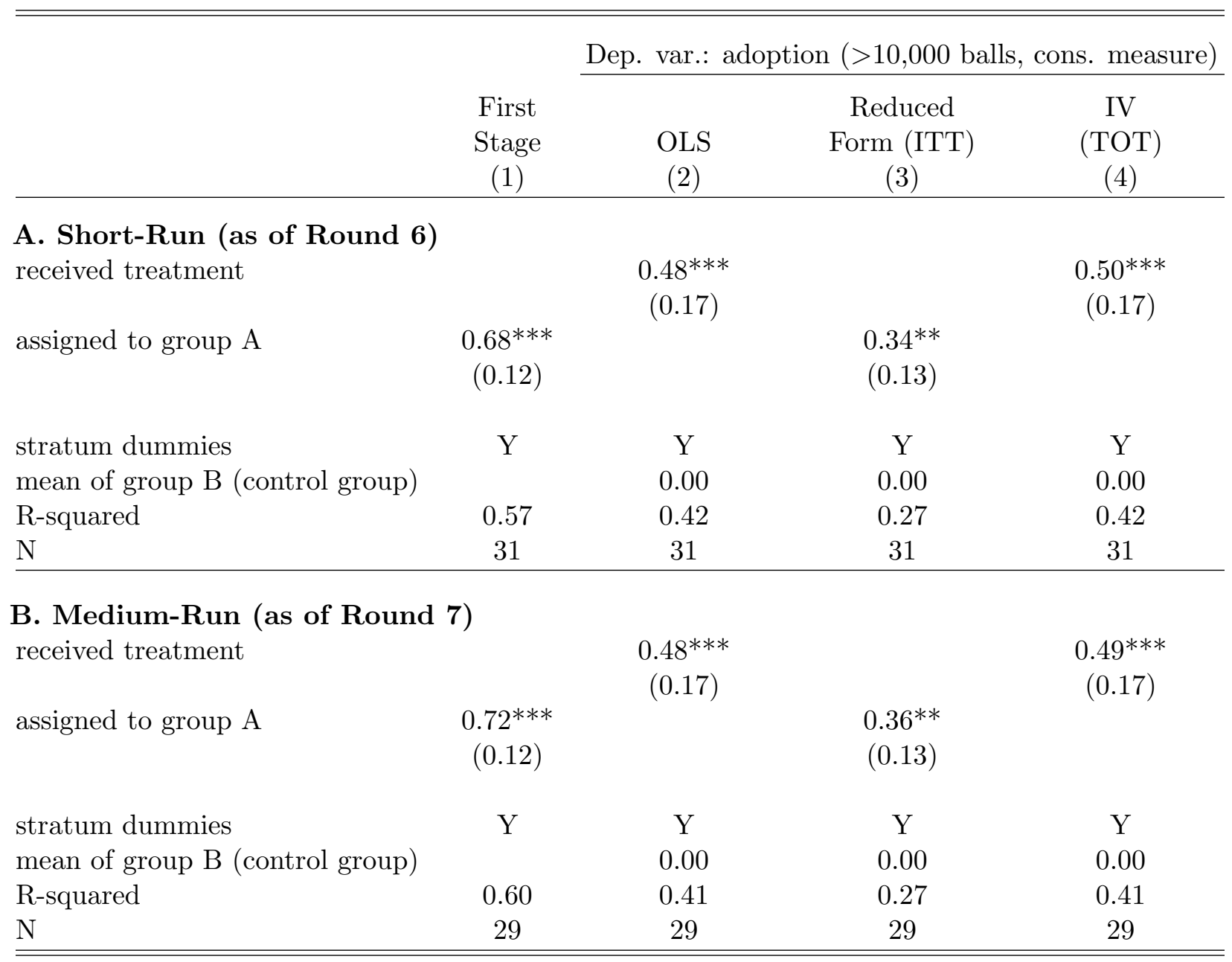

Notes: Table similar to Table 9 in main text but using 10,000-ball cutoff in conservative definition of adoption. Panel A reports short-run results as of Round 6 (Jan.-March 2014). Panel B reports medium-run results as of Round 7 (Oct.-Dec. 2014). The dependent variable in Column 1 is an indicator variable for whether the firm received treatment. Two firms exited between Rounds 6 and 7. All regressions include stratum dummies, and report robust standard errors. Significance: $* 0.10, * * 0.05 ; * * * 0.01$. 
Table A.9: Incentive-Payment Experiment (20,000-ball cutoff)

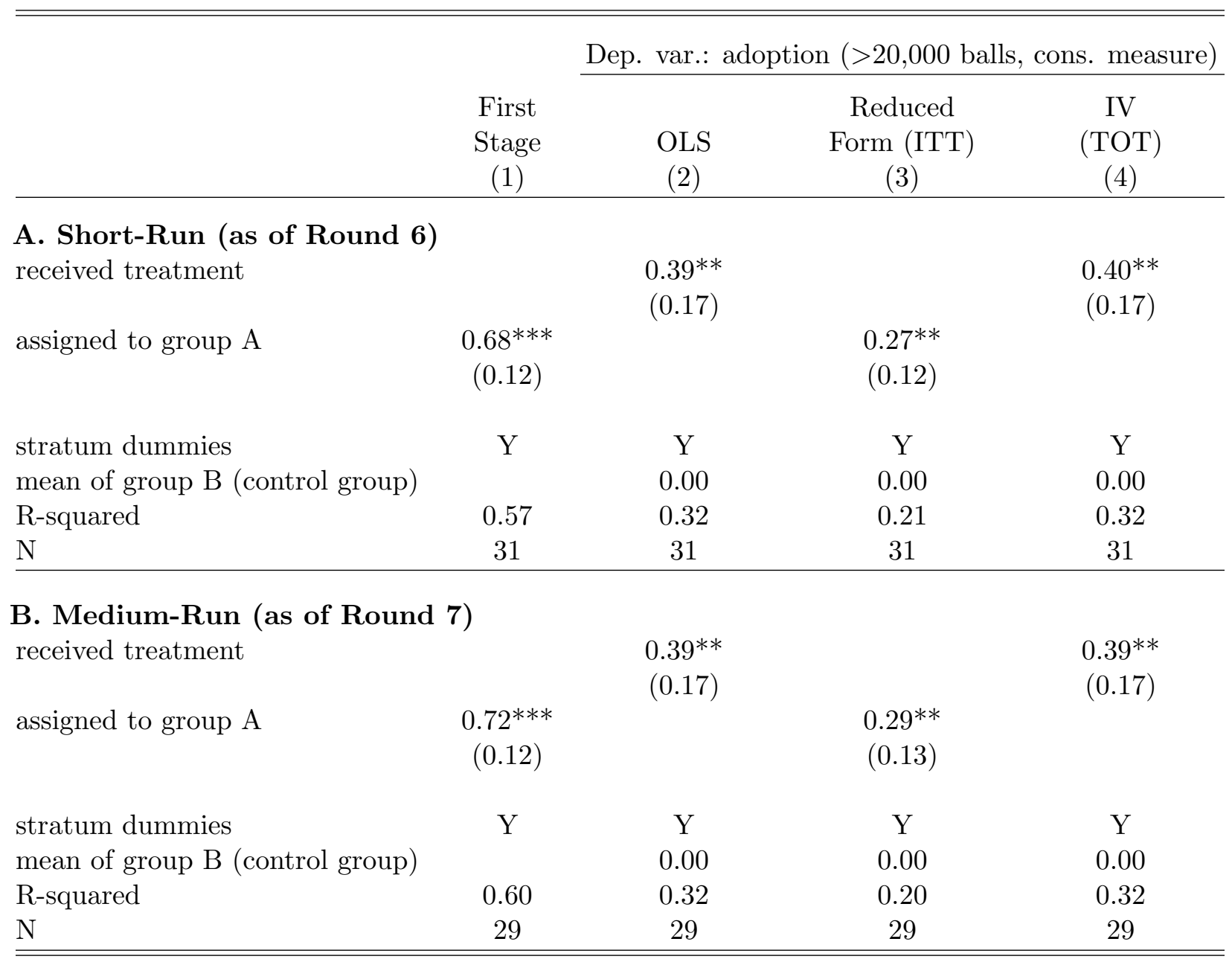

Notes: Table similar to Table 9 in main text but using 20,000-ball cutoff in conservative definition of adoption. Panel A reports short-run results as of Round 6 (Jan.-March 2014). Panel B reports medium-run results as of Round 7 (Oct.-Dec. 2014). The dependent variable in Column 1 is an indicator variable for whether the firm received treatment. Two firms exited between Rounds 6 and 7. All regressions include stratum dummies, and report robust standard errors. Significance: * $0.10, * * 0.05 ; * * * 0.01$. 
Table A.10: Wage Changes from August 2013 to September 2014

\begin{tabular}{lccc}
\hline \hline & $\begin{array}{c}\text { No Change } \\
(1)\end{array}$ & $\begin{array}{c}\text { Change } \\
(2)\end{array}$ & $\begin{array}{c}\text { Total Firms } \\
(3)\end{array}$ \\
\cline { 2 - 4 } A. Owner Responses & 10 & 14 & 24 \\
Head Cutter & 2 & 6 & 8 \\
Other Cutters & 13 & 11 & 24 \\
Head Printer & 10 & 6 & 16 \\
Other Printers & & & 15 \\
B. Employee Responses & 13 & 2 & 17 \\
Head Cutters (Self-Reported) & 13 & 4 & \\
Head Printers (Self-Reported) & & & \\
\hline \hline
\end{tabular}

Notes: Table reports the number of firms that made changes to wages between August 2013 and September 2014. All changes are increases. Panel A reports responses by the firm owner. Panel B reports self-reported responses by the head cutters and head printers. These data were collected in Round 7 of our survey. 
Table A.11: Reasons for Changing Payments

\begin{tabular}{lcccc}
\hline \hline & $\begin{array}{c}\text { Head Cutter } \\
(1)\end{array}$ & $\begin{array}{c}\text { Other } \\
\text { Cutters } \\
(2)\end{array}$ & $\begin{array}{c}\text { Head Printer } \\
(3)\end{array}$ & $\begin{array}{c}\text { Other } \\
\text { Printers } \\
(4)\end{array}$ \\
\cline { 2 - 5 } Because of Offset Die & 1 & 1 & 0 & 0 \\
New Hire & 1 & 0 & 0 & 0 \\
Worker Shortage & 0 & 0 & 0 & 0 \\
Prices were increasing & 3 & 0 & 1 & 1 \\
End of year change & 4 & 2 & 1 & 1 \\
Other & 1 & 1 & 2 & 3 \\
\hline Total & 10 & 4 & 4 & \\
\hline \hline
\end{tabular}

Notes: Table reports the owners' reasons for changing wages of employees between August 2013 and September 2014. These data were collected in Round 7 of our survey.

Table A.12: Why Owners Do Not Suggest Changes to Incentives

\begin{tabular}{lc}
\hline \hline & Total \\
\cline { 2 - 2 } I did not think about offering an incentive & 3 \\
Offering incentives to workers beyond their current piece rate is not common & 2 \\
I thought about offering an incentive, but the benefits of adoption were not high enough & 1 \\
If I offered an incentive to some workers, other workers would perceive this to be unfair & 3 \\
If I offered an incentive, workers would expect additional incentives for other tasks & 6 \\
Even if I had offered an incentive, the workers would not have adopted the offset die & 0 \\
Other & 3 \\
Total & 18 \\
\hline \hline
\end{tabular}

Notes: Table reports owners' self-reports about why they do not offer incentives to use the offset die. The owners were asked to choose from the list of reasons reported in the table. These data were collected in Round 7 of our survey. 


\section{Table A.13: Why Head Cutters Do Not Suggest Changes to Incentives}

\begin{tabular}{lc}
\hline \hline & Total \\
\cline { 2 - 2 } I did not think any changes in payment scheme were needed. & 0 \\
It is not my place to make suggestions about the payment scheme. & 11 \\
Management unlikely to listen to a suggestion from me about the payment scheme. & 0 \\
Suggesting would make firm more likely to adopt and my income would decline. & 1 \\
Other & 2 \\
Total & 14 \\
\hline \hline
\end{tabular}

Notes: Table reports the head cutters' self-reports about why they did not suggest making changes to the payment scheme to adopt the offset die. The cutters were asked to choose from the list of reasons reported in the table. These data were collected in Round 7 of our survey. 
Table A.14: Conversations about Changes to Payments

\section{A. Owners' reports of conversations about changing payment schemes}

\begin{tabular}{lccc} 
& $\begin{array}{c}\text { Head } \\
\text { Cutter } \\
\text { Yes }\end{array}$ & $\begin{array}{c}\text { Other } \\
\text { Cutters } \\
(2)\end{array}$ & $\begin{array}{c}\text { Head } \\
\text { Printer } \\
(3)\end{array}$ \\
\cline { 2 - 4 } No & 1 & 1 & 1 \\
Not Applicable & 21 & 7 & 21 \\
Total & 0 & 14 & 0 \\
\hline
\end{tabular}

B. Head cutters' reports of conversations about changing payment schemes

$\begin{array}{ccc} & \text { Head } & \text { Other } \\ \text { Owner } & \text { Printer } & \text { Cutters }\end{array}$

Yes

0

0

0

No

14

14

7

Not Applicable

0

0

7

Total

14

14

14

Notes: Table reports the answers to the question: "Did you discuss with any of the following people that the firm's payment scheme should be changed if the new offset die is adopted?" Panel A reports responses by the owner with the person indicated at the top of each column. Panel B reports responses by the head cutter. "Not applicable" means that the firm did not have an employee in the indicated category. These data were collected in Round 7 of our survey. 


\section{Table A.15: Owners' Reports of Conversations about the Offset Die}

\begin{tabular}{lccc}
\hline \hline & $\begin{array}{c}\text { Head Cutter } \\
(1)\end{array}$ & $\begin{array}{c}\text { Other Cutters } \\
(2)\end{array}$ & $\begin{array}{c}\text { Head Printer } \\
(3)\end{array}$ \\
\cline { 2 - 4 } Yes & 10 & 6 & 6 \\
No & 12 & 2 & 16 \\
Not Applicable & 0 & 14 & 0 \\
Total & & & 22 \\
\hline \hline
\end{tabular}

Notes: Table reports owners' answers to the question: "Did you have a conversation with this employee about whether you should adopt the offset die?" "Not applicable" means that the firm did not have an employee in the indicated category. These data were collected in Round 7 of our survey.

Table A.16: Cutters' Die Recommendation and Adoption

\begin{tabular}{lcc}
\hline \hline & \multicolumn{2}{c}{ Owner's decision } \\
\cline { 2 - 3 } Cutter's recommendation & $\begin{array}{c}\text { Did } \\
\text { Not Adopt } \\
(1)\end{array}$ & $\begin{array}{c}\text { Adopted } \\
(2)\end{array}$ \\
\hline Offset die is beneficial \& should be adopted & 0 & 3 \\
Offset die is not beneficial \& should not be adopted & 4 & 2 \\
Not sure whether the die is beneficial or not & 0 & 1 \\
Total & & 6 \\
\hline \hline
\end{tabular}

Notes: Table shows owners' reports of recommendations by head cutters about the offset die. "Did Not Adopt" indicates that the firm did not adopt the offset die according to the liberal definition, and "Adopt" indicates that the firm adopted. The total number of responses match the number of "yes" responses reported in Column 2 of Table A.15. These data were collected in Round 7 of our survey. 


\section{Table A.17: Adoption Speed of "Back-to-Back" Die}

\begin{tabular}{lc}
\hline \hline & $\begin{array}{c}\text { Number of } \\
\text { responses }\end{array}$ \\
\cline { 2 - 2 } Adopted when firm was born & 1 \\
Within a Month & 3 \\
1 Month & 7 \\
3 Months & 2 \\
6 Months & 1 \\
$>6$ Months & 0 \\
Total & 14 \\
\hline \hline
\end{tabular}

Notes: Table shows owners reports how quickly their firm adopted the two-panel non-offset "back-to-back" pentagon die after they first heard about the die. These data were collected in Round 7 of our survey.

Table A.18: Resistance to "Back-to-Back" Die

\begin{tabular}{|c|c|c|}
\hline & \multicolumn{2}{|c|}{ Resistance encountered from } \\
\hline & $\begin{array}{c}\text { Cutters } \\
(1)\end{array}$ & $\begin{array}{c}\text { Printers } \\
(2)\end{array}$ \\
\hline Yes & 1 & 1 \\
\hline No & 23 & 23 \\
\hline Total & 24 & 24 \\
\hline
\end{tabular}

Notes: Table shows owners' reports about whether firms encountered resistance from cutters and printers to adopting the two-panel non-offset "back-to-back" pentagon die. These data were collected in Round 7 of our survey. 
Table A.19: Payment Changes after Adoption of "Back-to-Back" Die

Number of

responses

Piece rate increased

1

Piece rate decreased 1

$\begin{array}{ll}\text { No change } & 19\end{array}$

Other type of change $\quad 3$

$\begin{array}{lr}\text { Total } & 24\end{array}$

Notes: Table reports the types of changes (if any) that firms made to payments when adopting the "back-to-back" pentagon die. These data were collected in Round 7 of our survey. 


\title{
Organizational Barriers to Technology Adoption: Evidence from Soccer-Ball Producers in Pakistan
}

\author{
David Atkin, Azam Chaudhry, Shamyla Chaudry
}

Amit K. Khandelwal and Eric Verhoogen

July 2016

Appendix B: Theory Appendix 


\section{B A Model of Organizational Barriers to Technology Adoption}

This appendix develops a model of strategic communication in a principal-agent setting that captures the intra-firm dynamics we have observed and motivates our second experiment, as discussed in Section 5 of the main text. After describing the theoretical setting in Section B.1, we consider the case in which the principal is not able to offer conditional contracts in Section B.2, and the case in which she is able to offer such contracts in Section B.3. In Section B.4, we present an additional result that a one-time lump-sum transfer can generate an equilibrium similar to the conditional-contracts case.

\section{B.1 Theoretical setting}

\section{B.1.1 Basic set-up}

Consider a one-period game. There is a principal (she) and an agent (he). The principal can sell any output $q$ at an exogenously given price $p$. The principal incurs two costs: a constant marginal cost of materials, $c$, and a wage $w(q)$ that she pays to the agent. Her payoff is $\pi=p q-w(q)-c q$. The agent produces output $q=s e$ where $s$ is the speed of the technology (e.g. the cuts per minute), and $e$ is effort, which is not contractible and is costly to the agent to provide. The agent has utility $U=w(q)-\frac{e^{2}}{2}$ and an outside option of zero. We assume that contracts must be of the linear form $w(q)=\alpha+\beta q$, where $\beta \geq 0 .{ }^{1}$ We further assume that the agent has limited liability, $\alpha \geq 0-\mathrm{a}$ reasonable assumption given that no worker in our setting pays an owner to work in the factory. Below we will consider cases which differ in the ability of the principal to condition the piece rate, $\beta$, on marginal cost, $c$, a characteristic of the technology that will in general only be revealed ex post.

Technologies are characterized by speed, $s$, and materials cost, $c$. There is an existing technology, $\theta_{0}$, with $\left(s_{0}, c_{0}\right)$. There is also a new technology, which can be one of three types:

- Type $\theta_{1}$, with $c_{1}=c_{0}$ and $s_{1}<s_{0}$. This technology is material-neutral (neither raises nor lowers material costs) and labor-using (is slower). It is dominated by the existing technology; we refer to it as the "bad" technology.

- Type $\theta_{2}$, with $c_{2}<c_{0}$ and $s_{2}<s_{0}$. This technology is material-saving but labor-using: it lowers material costs but is slower than the existing technology. It is analogous to our offset die.

- Type $\theta_{3}$, with $c_{3}=c_{0}$ and $s_{3}>s_{0}$. This technology is material-neutral and labor-saving. It dominates the existing technology because it has the same material costs but is faster. It is analogous to the original two-panel non-offset "back-to-back" die (faster than the one-piece die that preceded it) discussed in Section 7.

We assume that both players are aware ex ante of the existence of the technology, but differ in their knowledge about the technology type. The principal has prior $\rho_{i}$ that the technology is type $\theta_{i}$, with $\sum_{1}^{3} \rho_{i}=1$, and Nature does not reveal the type to her. In contrast, Nature reveals the type with certainty to the agent. While this is clearly an extreme assumption, it captures in an analytically

\footnotetext{
${ }^{1}$ We restrict attention to a single contract rather than a menu of contracts since there was no evidence such menus were on offer in Sialkot. Also, we rule out by assumption the possibility that the contract can be conditioned on messages sent by the agent, implicitly assuming that the costs of implementing such contracts are prohibitively high. There is an active theoretical literature on optimal contracts in settings similar to ours, in which agents need to be induced to experiment; see e.g. Halac, Kartik, and Liu (2016).
} 
tractable way the observation from our qualitative work that the cutters are better informed about the cutting dies (which they work with all day every day) than are owners. Adopting the new technology requires a fixed cost, $F$.

In Stage 1 of the game, the principal chooses a wage contract. In Stage 2, Nature reveals the technology type to the agent. In Stage 3, the agent can send a costless message regarding the type of the new technology. In Stage 4, the principal decides whether to adopt the new technology, given the agent's message. In Stage 5, the agent chooses his level of effort. In Stage 6, output is observed, the technology is revealed to the principal, and payoffs are realized. The key feature of the timing is that the wage contract must be chosen before the agent sends his message..$^{2,3}$

Given this setup, the optimal effort choice for the agent, for a given $\beta$, is:

$$
e=\underset{e}{\arg \max }\left(\alpha+\beta s_{i} e-\frac{e^{2}}{2}\right)=\beta s_{i}
$$

In all the cases we consider below, the limited-liability constraint binds and the principal will set $\alpha=0$. Conditional on technology $\theta_{i}$ being used, the agent's utility is then:

$$
U\left(\beta, \theta_{i}\right)=\frac{\beta^{2} s_{i}^{2}}{2}
$$

which makes it clear that conditional on $\beta$ the agent prefers faster technologies. Given the agent's optimal effort choice, the principal's profit from adopting technology type $\theta_{i}$ can be written as a function of the piece rate, $\beta$. Writing $\pi\left(\beta, \theta_{i}\right)$ as $\pi_{i}(\beta)$ to reduce clutter, we have:

$$
\pi_{i}(\beta)=s_{i}^{2} \beta\left(p-\beta-c_{i}\right)-F \cdot \mathbb{1}(i=1,2,3)
$$

where $\beta$ need not be the optimal choice for technology $\theta_{i}$.

\section{B.1.2 Benchmarks}

As a preliminary step, it is useful to consider the optimal contract under two counterfactual benchmark cases. In the first, suppose that the principal is fully informed about the technology. In this case, in the absence of the limited-liability constraint the principal would make the agent the residual claimant: she would set $\beta=p-c_{i}$ and bring the agent down to his reservation utility through a negative value of $\alpha$. With the limited-liability constraint this is not possible. Since the

\footnotetext{
${ }^{2}$ Since Nature does not reveal the technology type to the principal, it is not crucial for the analysis whether Nature's move, which we can think of as the initial technology drop by our survey team, happens before or after the wage contract is set. (That is, the order of Stages 1 and 2 can be reversed.) Thus the model can also accommodate a scenario in which the principal's priors are set when our survey team does the technology drop and the technology type is revealed to the agent.

${ }^{3}$ One concern with static cheap-talk models is that the principal has no chance to respond to lying, and hence no way to encourage truth telling, if she later discovers that the technology is a good one (for example, from another firm that adopts). A more general version of our model would partially address this concern. Consider the following modification to Stage 2 of the game: if the technology is type $\theta_{3}$, Nature signals $\theta_{3}$ to the agent; if the technology is type $\theta_{2}$, Nature signals $\theta_{2}$ to the agent with probability $\varphi>\frac{1}{2}$ and $\theta_{1}$ with probability $1-\varphi$; and if the technology is type $\theta_{1}$, Nature signals $\theta_{1}$ to the agent with probability $\varphi>\frac{1}{2}$ and $\theta_{2}$ with probability $1-\varphi$. (Recall that conditional on $\beta$ the agent's utility is only a function of $s_{i}$ and not $c_{i}$ so it is reasonable that he can more easily distinguish slow from fast technologies than low cost from high cost.) This leaves our model essentially unchanged since the agent still wishes to block adoption if he receives either signal $\theta_{1}$ or $\theta_{2}$ and to encourage adoption if he receives signal $\theta_{3}$. Hence, he continues to pool types $\theta_{1}$ and $\theta_{2}$, and the principal anticipates this pooling. The key difference is that in this variant of the model, if the principal later discovers the technology is of type $\theta_{2}$, she cannot be sure that the agent received the signal $\theta_{2}$.
} 
agent's effort ( $e=\beta s_{i}$, as above) is independent of $\alpha$ (refer to (B1)), the principal sets $\alpha=0$. The optimal contract for a known technology type $\theta_{i}$ is then:

$$
\alpha_{i}=0, \beta_{i}=\frac{p-c_{i}}{2}
$$

Note that the optimal piece rate depends on marginal cost. ${ }^{4}$ The optimal piece rate for technology $\theta_{2}, \beta_{2}$, is higher than the optimal piece rate for the existing technology, $\beta_{0}$, since $c_{2}<c_{0} .{ }^{5}$ In this case, the principal would like to incentivize more effort from the agent because profits per cut are higher.

In the second benchmark, suppose that the principal is imperfectly informed but receives no message from the agent. Define expected profit in this case as:

$$
\widetilde{\pi}(\beta) \equiv \rho_{1} \pi_{1}(\beta)+\rho_{2} \pi_{2}(\beta)+\rho_{3} \pi_{3}(\beta)
$$

In this case, it can be shown that the principal, if she were to adopt the technology, would choose the wage contract: $\alpha=0, \widetilde{\beta}=\sum_{i=1}^{3} \lambda_{i} \beta_{i}$, where $\beta_{i}$ is as in (B4) and $\lambda_{i}=\frac{\rho_{i} s_{i}^{2}}{\sum_{1}^{3} \rho_{i} s_{i}^{2}}$. The optimal piece rate would thus be a weighted average of the optimal piece rates in the full-information case. Given this contract, the expected profit from adoption would be:

$$
\widetilde{\pi}(\widetilde{\beta})=\left(\sum_{i=1}^{3} \rho_{i} s_{i}^{2}\right)(\widetilde{\beta})^{2}-F
$$

\section{B.1.3 Parameter restrictions}

As noted above, the aim of the model is to capture the intra-organizational dynamics we have observed, in particular that workers may seek to discourage owners from adopting a technology like ours, and that modifying wage contracts may lead to successful adoption. These features are not present under all possible parameter values. To focus on what we consider to be the interesting case in the model, we impose three parameter restrictions. Using the definitions of $\pi_{i}(\cdot)$ from $(\mathrm{B} 3)$, of $\beta_{i}$ from (B4), and of $\widetilde{\pi}(\widetilde{\beta})$ from (B6), they are:

$$
\begin{gathered}
\pi_{2}\left(\beta_{0}\right)>\pi_{0}\left(\beta_{0}\right) \\
\pi_{3}\left(\beta_{2}\right)>\pi_{0}\left(\beta_{2}\right) \\
\pi_{0}\left(\beta_{0}\right)>\widetilde{\pi}(\widetilde{\beta})
\end{gathered}
$$

To understand the intuition for these conditions, consider the schematic representation of the

\footnotetext{
${ }^{4}$ There are alternative scenarios where the piece rate would depend not only on the price and marginal cost, but also on speed. As a first example, if speed and effort were substitutes, $q=s+e$, then agents will optimally provide effort $e=\beta$ and the optimal piece rate for a given technology is $\beta=\frac{p-c_{i}-s_{i}}{2}$. However, the agent's utility conditional on $\beta, U\left(\beta, \theta_{i}\right)=\frac{\beta^{2}}{2}+\beta s_{i}$, is still increasing in speed. Since wages are set in Stage 1, the agent will still try to avoid slower technologies like type $\theta_{2}$ in Stage 3 . Whether the principal will want to adopt types $\theta_{2}$ and $\theta_{3}$ will again depend on parameters and there will still be a potential misalignment in incentives. Second, suppose that the principal has to produce a target $\bar{q}$ with no time limit and, as in our model, $q=$ se and optimal effort for technology type $\theta_{i}$ is $\beta s_{i}$. Since $q=\beta s_{i}^{2}$, the optimal piece rate is to pay $\beta=\frac{\bar{q}}{s_{i}^{2}}$ to hit the target (assuming this is less than $\beta=\frac{p-c}{2}$, otherwise she would just hire several cutters at this optimal piece rate). But since the agent's utility conditional on $\beta$ is still increasing in $s, U\left(\beta, \theta_{i}\right)=\frac{\beta^{2} s_{i}^{2}}{2}$, the agent will still try to avoid slower technologies like type $\theta_{2}$ creating a potential misalignment in incentives.

${ }^{5}$ Since $c_{3}=c_{1}=c_{0}$, the optimal piece rate for types $\theta_{1}$ and $\theta_{3}$ is the same as for the existing technology.
} 
profit functions $\pi_{i}(\beta)$ in Figure B.1. Each of the profit functions $\pi_{i}(\beta)$ defines a concave parabola with vertex at $\beta_{i}$. ${ }^{6}$ Since $c_{0}=c_{1}=c_{3}$, the functions $\pi_{0}(\beta), \pi_{1}(\beta)$, and $\pi_{3}(\beta)$ have maxima at the same value of the piece rate, $\beta_{0} ; \pi_{2}(\beta)$ has a maximum at $\beta_{2}>\beta_{0}$.

Condition (B7a) requires that $\pi_{2}(\beta)$ lie above $\pi_{0}(\beta)$ even at the optimal piece rate for the existing technology $\beta_{0}$, as in the figure. We believe that this condition is realistic in our empirical setting, where our technology is profitable to adopt for almost all firms even at existing wage rates. The condition in turn implies $\pi_{2}\left(\beta_{2}\right)>\pi_{0}\left(\beta_{0}\right)$; that is, a fully informed principal would adopt type $\theta_{2}$.

Condition $(\mathrm{B} 7 \mathrm{~b})$ requires that technology $\theta_{3}$ dominates the existing technology $\theta_{0}$ even at $\beta_{2}$, the optimal piece rate for type $\theta_{2}$. This condition guarantees that $\theta_{3}$ dominates $\theta_{0}$ at all values of the piece rate between $\beta_{0}$ and $\beta_{2}$, which will be the region of primary interest. ${ }^{7}$

Condition (B7c) implies that a principal with no information beyond her priors would choose not to adopt. Intuitively, it requires that the payoff to the bad technology $\theta_{1}$ is sufficiently low and that the principal's prior on $\theta_{1}$ is sufficiently high that the $\widetilde{\pi}(\widetilde{\beta})$ curve defined in (B6) (a weighted average of $\pi_{1}(\beta), \pi_{2}(\beta)$, and $\pi_{3}(\beta)$, and itself a concave parabola) lies everywhere below $\pi_{0}\left(\beta_{0}\right)$.

Remarks 1-3 in Appendix B.5 show formally that conditions (B7a)-(B7c) imply that the relative locations of the $\pi_{i}(\beta)$ curves are as illustrated in Figure B.1. ${ }^{8}$ Remark 4 shows formally that conditions (B7a)-(B7c) are compatible with each other.

\section{B.1.4 Cheap-talk Subgames Conditional on $\beta$}

For a given choice of the piece rate $\beta$ by the principal, the agent and principal engage in a cheaptalk subgame similar to the interaction considered by Crawford and Sobel (1982) (hereafter CS) but with a discrete set of possible states of the world. Let $\Theta$ be the set of possible new technologies (i.e. $\left.\left\{\theta_{1}, \theta_{2}, \theta_{3}\right\}\right)$. We refer to an agent who observes $\theta_{i}$ as being "type $\theta_{i}$." Let $m$ be a message and $M$ the set of possible messages. Let $q(m \mid \theta)$ be the agent's probability of sending message $m$ if he is type $\theta$, where $\sum_{m \in M} q(m \mid \theta)=1$ for each $\theta$. Let $\rho(\theta)$ be the principal's prior distribution; that is, $\rho\left(\theta_{1}\right)=\rho_{1}, \rho\left(\theta_{2}\right)=\rho_{2}, \rho\left(\theta_{3}\right)=\rho_{3}$. Let $a(m) \in[0,1]$ be the probability of adoption by the principal in response to the message $m$. Let $\widehat{U}\left(a(m), \beta, \theta_{i}\right)$ be the expected utility of the agent of type $\theta_{i}$, prior to the adoption decision of the principal:

$$
\widehat{U}\left(a(m), \beta, \theta_{i}\right)=a(m) U\left(\beta, \theta_{i}\right)+(1-a(m)) U\left(\beta, \theta_{0}\right)
$$

where $U(\cdot, \cdot)$ is as defined in (B2). Define $\widehat{\pi}\left(a, \beta, \theta_{i}\right)$ as the expected profit of the principal prior to the adoption decision, conditional on $\theta_{i}$ :

$$
\widehat{\pi}\left(a, \beta, \theta_{i}\right)=a \pi_{i}(\beta)+(1-a) \pi_{0}(\beta)
$$

${ }^{6}$ To see this, note that (B3) can be rewritten:

$$
\pi_{i}(\beta)=-s_{i}^{2}\left(\beta-\beta_{i}\right)^{2}+s_{i}^{2} \beta_{i}^{2}-F \cdot \mathbb{1}(i=1,2,3)
$$

where $\beta_{i}$ is as defined in (B4). The width of each parabola is declining in speed, $s_{i}$.

${ }^{7}$ As piece rates rise towards $p-c_{0}$, operating profits fall to zero for both the existing technology and type $\theta_{3}$ and so the existing technology, which requires no fixed cost $F$ of adoption, becomes preferred to $\theta_{3}$. Thus, the condition will be satisfied by some combination of large speed gains from $\theta_{3}$, low fixed costs of adoption for $\theta_{3}$, and small marginal cost gains from $\theta_{2}$.

${ }^{8}$ Note that the conditions do not carry an implication for the relative magnitudes of $\pi_{3}\left(\beta_{0}\right)$ and $\pi_{2}\left(\beta_{2}\right)$; it may be that $\pi_{3}\left(\beta_{0}\right)>\pi_{2}\left(\beta_{2}\right)$ as in the figure, or that $\pi_{3}\left(\beta_{0}\right)<\pi_{2}\left(\beta_{2}\right)$. 
where $\pi_{i}(\cdot)$ is as defined in in (B3). The principal's posterior beliefs after receiving message $m$, by Bayes' rule, are:

$$
p(\theta \mid m)=\frac{q(m \mid \theta) \rho(\theta)}{\sum_{\theta^{\prime} \in \Theta} q\left(m \mid \theta^{\prime}\right) \rho\left(\theta^{\prime}\right)}
$$

An equilibrium in the cheap-talk subgame is a family of reporting rules $q(m \mid \theta)$ for the agent (sender) and an action rule $a(m)$ for the principal (receiver) such that the following conditions hold:

1. If $q\left(m^{*} \mid \theta\right)>0$ then

$$
m^{*}=\underset{m \in M}{\arg \max } \widehat{U}(a(m), \beta, \theta)
$$

2. For each $m$,

$$
a(m)=\underset{a \in[0,1]}{\arg \max } \sum_{\theta \in \Theta} \widehat{\pi}(a, \beta, \theta) p(\theta \mid m)
$$

That is, the rule of each player must be a best response to the rule of the other player.

Following CS, we describe two messages $m$ and $m^{\prime}$ as equivalent in a given subgame equilibrium if they induce the same action, that is $a(m)=a\left(m^{\prime}\right)$. Let $M_{a}=\{m: a(m)=a\}$ be the set of equivalent messages that lead the principal to choose action $a$. Following CS, we say that an action $a$ is induced by an agent of type $\theta$ if $\sum_{m \in M_{a}} q(m \mid \theta)>0$. For a given subgame equilibrium, let $m_{\text {min }} \equiv \arg \min _{m \in M} a(m)$ and $m_{\max } \equiv \arg \max _{m \in M} a(m)$ be messages that induce the lowest and highest probabilities of adoption, respectively. Let $a_{\min } \equiv a\left(m_{\min }\right)$ and $a_{\max } \equiv a\left(m_{\max }\right)$ be the corresponding lowest and highest induced probabilities of adoption, and $M_{a_{\min }}$ and $M_{a_{\max }}$ be the sets of equivalent messages that induce them.

To streamline the exposition, we treat each set $M_{a}$ as a single message and treat subgame equilibria that differ only in which messages from a set $M_{a}$ are chosen as the same subgame equilibrium. We refer to $M_{1}$ as "technology is good" and $M_{0}$ as "technology is bad." 9

\section{B.2 No conditional contracts}

We first consider the case in which the (imperfectly informed) principal is not able to condition the wage payment on marginal cost, which is only revealed ex post. In this case, there exists an equilibrium in which, if the technology is type $\theta_{2}$, the agent seeks to discourage the principal from adopting and the principal does not adopt. If we restrict attention to the most informative equilibria in all cheap-talk interactions, then this equilibrium is unique. For conciseness, the following proposition only states the on-equilibrium-path strategies; in the proof below we consider the entire strategy space.

Proposition 1. Under $(B \% a)-(B \% c)$, if contracts conditioned on marginal cost are not available, then the following strategies are part of a perfect Bayesian equilibrium.

1. In Stage 1, the principal offers wage contract $\left(\alpha^{*}=0, \beta^{*}=\beta_{0}=\frac{p-c_{0}}{2}\right)$.

2. In Stage 3, the agent:

(a) says "technology is bad" if the technology is type $\theta_{1}$ or $\theta_{2}$,

\footnotetext{
${ }^{9}$ If we were to limit the set of messages to have just three elements, $m_{1}=$ "the techology is type $\theta_{1}$ ", $m_{2}=$ "the techology is type $\theta_{2}$ ", and $m_{3}=$ "the techology is type $\theta_{3}$ ", then a natural subgame equilibrium would have $m_{1} \in M_{0}$ and $m_{2}, m_{3} \in M_{1}$; that is, to discourage adoption the agent would say that the technology is type $\theta_{1}$ and to encourage it he would say type $\theta_{2}$ or $\theta_{3}$. Since formally there is no need to limit the set of messages in this way, we consider the richer set of potential messages in the proofs below.
} 
(b) says "technology is good" if the technology is type $\theta_{3}$.

3. In Stage 4, the principal:

(a) adopts if the agent says "technology is good",

(b) does not adopt if the agent says "technology is bad".

Intuitively, given that the principal has committed in Stage 1 to a piece rate (not conditioned on cost), the agent strictly prefers the existing technology to type $\theta_{2}$. So if the technology is type $\theta_{2}$, the agent discourages adoption, and the principal does not adopt. ${ }^{10}$ Why does the principal pay any attention to the agent's message, given that she knows that the agent does not want to adopt type $\theta_{2}$ ? The intuition is that the players' interests are aligned if the technology is of type $\theta_{1}$ or $\theta_{3}$, and the agent's advice is valuable enough in these states of the world that it is worthwhile for the principal to allow herself to be influenced by the agent - and possibly discouraged from using type $\theta_{2}$ - rather than to ignore the agent's advice altogether.

\section{B.2.1 Proof of Proposition 1}

To prove this proposition, we first consider the cheap-talk interaction in the subgame defined by any choice of piece rate, $\beta$ (Subsection B.2.1.1). We then consider the particular subgame defined by a particular choice of $\beta$, namely $\beta=\beta_{0}$ (Subsection B.2.1.2). We then show that this choice is optimal for the principal (Subsection B.2.1.3).

\section{B.2.1.1 Subgames conditional on $\beta$}

For the subgame corresponding to any choice of $\beta$, we have the following.

Lemma 1. In any equilibrium with $a_{\min }<a_{\max }$ the following statements are true:

$$
\begin{aligned}
& \sum_{m \in M_{a_{m i n}}} q\left(m \mid \theta_{1}\right)=1 \quad \sum_{m \in M_{a_{m a x}}} q\left(m \mid \theta_{1}\right)=0 \quad \sum_{m \notin\left\{M_{a_{m i n}} \cup M_{a_{m a x}}\right\}} q\left(m \mid \theta_{1}\right)=0 \\
& \sum_{m \in M_{a_{m i n}}} q\left(m \mid \theta_{2}\right)=1 \quad \sum_{m \in M_{a_{m a x}}} q\left(m \mid \theta_{2}\right)=0 \quad \sum_{m \notin\left\{M_{a_{m i n}} \cup M_{a_{m a x}}\right\}} q\left(m \mid \theta_{2}\right)=0 \\
& \sum_{m \in M_{a_{m i n}}} q\left(m \mid \theta_{3}\right)=0 \quad \sum_{m \in M_{a_{m a x}}} q\left(m \mid \theta_{3}\right)=1 \quad \sum_{m \notin\left\{M_{a_{m i n}} \cup M_{a_{m a x}}\right\}} q\left(m \mid \theta_{3}\right)=0
\end{aligned}
$$

For $m \in M_{a_{\text {min }}}, p\left(\theta_{1} \mid m\right)=\frac{\rho_{1}}{\rho_{1}+\rho_{2}}, p\left(\theta_{2} \mid m\right)=\frac{\rho_{2}}{\rho_{1}+\rho_{2}}$, and $p\left(\theta_{3} \mid m\right)=0$. For $m \in M_{a_{\text {max }}}, p\left(\theta_{1} \mid m\right)=$ $0, p\left(\theta_{2} \mid m\right)=0$, and $p\left(\theta_{3} \mid m\right)=1$.

In any equilibrium with $a_{\min }=a_{\max }$ (letting $a^{*} \equiv a_{\min }=a_{\max }$ ), the following statements are true:

$$
\sum_{m \in M_{a^{*}}} q\left(m \mid \theta_{i}\right)=1 \forall \theta_{i}, \quad \sum_{m \notin M_{a^{*}}} q\left(m \mid \theta_{i}\right)=0 \forall \theta_{i}
$$

For $m \in M_{a^{*}}, p\left(\theta_{1} \mid m\right)=\rho_{1}, p\left(\theta_{2} \mid m\right)=\rho_{2}$, and $p\left(\theta_{3} \mid m\right)=\rho_{3}$.

Proof. Note from (B9) that $\frac{\partial}{\partial a} \widehat{U}\left(a, \beta, \theta_{1}\right)<0, \frac{\partial}{\partial a} \widehat{U}\left(a, \beta, \theta_{2}\right)<0$ and $\frac{\partial}{\partial a} \widehat{U}\left(a, \beta, \theta_{3}\right)>0$ for all $a \in[0,1]$, since $s_{1}<s_{0}, s_{2}<s_{0}$ and $s_{3}>s_{0}$. Hence if $a_{\min } \neq a_{\max }$ then in order for (B12) to

\footnotetext{
${ }^{10}$ In the language of Aghion and Tirole (1997), the principal in this equilibrium retains formal authority over the adoption decision but cedes real authority to the agent.
} 
be satisfied it must be the case that an agent of type $\theta_{1}$ or $\theta_{2}$ chooses a message that induces the lowest possible probability of adoption, $a_{\text {min }}$. Similarly, an agent who observes $\theta_{3}$ must choose a message that induces the highest possible probability of adoption, $a_{\max }$. Given these reporting rules, the updating of the principal's beliefs follow by Bayes' rule.

If $a_{\min }=a_{\max }$ then trivially all messages sent by the agent induce the same action and hence are equivalent $\left(M_{a_{\min }}=M_{a_{\max }}=M_{a^{*}}\right)$. Given this, the principal does not update.

Intuitively, conditional on a piece rate, $\beta$, types $\theta_{1}$ and $\theta_{2}$ strictly prefer non-adoption, and type $\theta_{3}$ strictly prefers adoption; the types report accordingly, with the most discouraging and most encouraging messages. So in any equilibrium in which the principal's action is influenced by the agent's message (i.e. $a_{\min }<a_{\max }$ ), if an agent sends a discouraging message the principal infers that he is type $\theta_{1}$ or $\theta_{2}$; if the message is encouraging, she infers that he is type $\theta_{3}$. She then updates by Bayes' rule.

The Lemma holds that for a given $\beta$, only two subgame equilibria (modulo treating messages that induce the same action as equivalent) may exist: one in which $a_{\min } \neq a_{\max }$ and agent types $\theta_{1}$ and $\theta_{2}$ are indistinguishable and another in which the principal ignores the message from the agent (a "babbling" equilibrium). In the terminology of Sobel (2013), an equilibrium is "informative" if the message from the sender shifts the receiver's beliefs and is "influential" if different messages induce the receiver to take different actions. By these definitions, the equilibrium with $a_{\min } \neq a_{\max }$ is both informative and influential.

It will be convenient below to define the principal's ex-ante expected profit in a given subgame equilibrium. Given Lemma 1, we have:

$$
\begin{aligned}
\pi^{*}(\beta)= & \rho_{1} \widehat{\pi}\left[a\left(m \mid m \in M_{a_{\min }}\right), \beta, \theta_{1}\right]+\rho_{2} \widehat{\pi}\left[a\left(m \mid m \in M_{a_{\min }}\right), \beta, \theta_{2}\right] \\
& +\rho_{3} \widehat{\pi}\left[a\left(m \mid m \in M_{a_{\max }}\right), \beta, \theta_{3}\right]
\end{aligned}
$$

where $a(m)$ represents the principal's best response, given by (B13), and we may or may not have $a_{\min }=a_{\max }$.

\section{B.2.1.2 Subgame with $\beta=\beta_{0}$}

Now consider the particular subgame with $\beta=\beta_{0}$.

Lemma 2. If $\beta=\beta_{0}$, there exists a perfect Bayesian subgame equilibrium with the strategies outlined in Proposition 1.

Proof. As outlined by Proposition 1, the agent's reporting rules are given by (B14a)-(B14c), where $a_{\min }=0$ and $a_{\max }=1$, and the principal's action rule is $a(m)=0 \forall m \in M_{0}, a(m)=1 \forall m \in$ $M_{1} \cdot{ }^{11}$ To prove that this is a subgame equilibrium, it suffices to show that neither agent nor principal wants to deviate. That the agent does not want to deviate follows from the fact (from (B9)) that $\frac{\partial}{\partial a} \widehat{U}\left(a, \beta, \theta_{1}\right)<0, \frac{\partial}{\partial a} \widehat{U}\left(a, \beta, \theta_{2}\right)<0$ and $\frac{\partial}{\partial a} \widehat{U}\left(a, \beta, \theta_{3}\right)>0$ for all $a \in[0,1]$. Now consider the principal's decision. Given Lemma 1 , if the agent says "technology is bad" (i.e. any $m \in M_{0}$ ) then the condition for the principal not to deviate is:

$$
\pi_{0}(\beta) \geq \frac{\rho_{1}}{\rho_{1}+\rho_{2}} \pi_{1}(\beta)+\frac{\rho_{2}}{\rho_{1}+\rho_{2}} \pi_{2}(\beta)
$$

\footnotetext{
${ }^{11}$ Note that in the Proposition-1 equilibrium all messages fall into one of the following sets: $M_{0}, M_{1}$, or the complement to $M_{0} \cup M_{1}$, no element of which is used in equilibrium.
} 
where the left-hand side is the profit from the existing technology and the right-hand side the expected profit to adoption. That this condition holds for $\beta=\beta_{0}$ is demonstrated by Remark 5 below.

If the agent says "technology is good" (i.e. any $m \in M_{1}$ ), then the condition for the principal not to deviate is:

$$
\pi_{0}(\beta) \leq \pi_{3}(\beta)
$$

which by Remark 2 is satisfied for $\beta=\beta_{0}$.

In this subgame, the principal's ex-ante expected profit (refer to (B16)) is:

$$
\begin{aligned}
\pi^{*}\left(\beta_{0}\right) & =\rho_{1} \widehat{\pi}\left(0, \beta_{0}, \theta_{1}\right)+\rho_{2} \widehat{\pi}\left(0, \beta_{0}, \theta_{2}\right)+\rho_{3} \widehat{\pi}\left(1, \beta_{0}, \theta_{3}\right) \\
& =\left(\rho_{1}+\rho_{2}\right) \pi_{0}\left(\beta_{0}\right)+\rho_{3} \pi_{3}\left(\beta_{0}\right)
\end{aligned}
$$

\section{B.2.1.3 Principal's choice of $\beta$}

We now turn to the supergame where the principal selects the optimal $\beta$ given the subgame payoffs. We can show that the principal has no incentive to deviate from $\beta_{0}$ in the supergame.

Lemma 3. The principal's expected payoff in the informative equilibrium of the subgame with piece rate $\beta_{0}$ is strictly greater than the payoffs in the subgames for all other possible values of $\beta$.

Proof. From Lemma 1, types $\theta_{1}$ and $\theta_{2}$ can never be distinguished. Therefore, the highest possible payoff to the principal from any subgame equals the payoff that the principal would obtain if she observed whether $\theta=\theta_{3}$ or $\theta \in\left\{\theta_{1}, \theta_{2}\right\} .{ }^{12}$ Consider the maximal payoffs in all subgames under the assumption that the principal is able to extract this information. There are only four cases to consider, which may exist for different values of $\beta .{ }^{13}$

1. It is profitable for the principal to adopt if $\theta=\theta_{3}$ but not if $\theta \in\left\{\theta_{1}, \theta_{2}\right\}$ :

$$
\begin{aligned}
\widehat{\pi}\left(1, \beta, \theta_{3}\right) & \geq \widehat{\pi}\left(0, \beta, \theta_{3}\right) \\
\frac{\rho_{1}}{\rho_{1}+\rho_{2}} \widehat{\pi}\left(1, \beta, \theta_{1}\right)+\frac{\rho_{2}}{\rho_{1}+\rho_{2}} \widehat{\pi}\left(1, \beta, \theta_{2}\right) & <\frac{\rho_{1}}{\rho_{1}+\rho_{2}} \widehat{\pi}\left(0, \beta, \theta_{1}\right)+\frac{\rho_{2}}{\rho_{1}+\rho_{2}} \widehat{\pi}\left(0, \beta, \theta_{2}\right)
\end{aligned}
$$

In this case, the principal's maximal ex-ante expected profit (refer to (B16)) is:

$$
\begin{aligned}
\pi^{*}(\beta) & =\rho_{1} \widehat{\pi}\left(0, \beta, \theta_{1}\right)+\rho_{2} \widehat{\pi}\left(0, \beta, \theta_{2}\right)+\rho_{3} \widehat{\pi}\left(1, \beta, \theta_{3}\right) \\
& =\left(\rho_{1}+\rho_{2}\right) \pi_{0}(\beta)+\rho_{3} \pi_{3}(\beta)
\end{aligned}
$$

By Remarks 1-3, this case holds when $\beta=\beta_{0}$. From the definition of $\pi_{i}(\cdot)$ in (B3) it follows immediately that the expected payoff is maximized at $\beta^{*}=\beta_{0} \equiv \frac{p-c_{0}}{2}$. That is, $\pi^{*}\left(\beta_{0}\right)>\pi^{*}(\beta) \forall \beta \neq \beta_{0}$. Hence for all subgames that fall into this case, the principal prefers $\beta_{0}$ to any other $\beta$.

\footnotetext{
${ }^{12}$ This is a simple application of Blackwell's ordering, i.e. that the decision maker's payoff must be weakly higher with more information (see Blackwell (1953).)

${ }^{13}$ The sets of values of $\beta$ for which the different cases hold depend on the values of the parameters $\hat{\hat{\beta}}_{3}$ and $\bar{\beta}$ defined in Remarks 2 and 5. There are two possibilities: (1) $\bar{\beta} \geq \hat{\hat{\beta}}_{3}$. Here the region $\left(0, \hat{\beta}_{3}\right)$ corresponds to Case 2 below, $\left[\hat{\beta}_{3}, \hat{\hat{\beta}}_{3}\right]$ to Case $1,\left[\hat{\hat{\beta}}_{3}, \bar{\beta}\right]$ to Case 2 , and $(\bar{\beta}, \infty)$ to Case 4 . (2) $\bar{\beta}<\hat{\hat{\beta}}_{3}$. Here the region $\left(0, \hat{\beta}_{3}\right)$ corresponds to Case 2 below, $\left[\hat{\beta}_{3}, \bar{\beta}\right)$ to Case $1,\left[\bar{\beta}, \hat{\hat{\beta}}_{3}\right]$ to Case 3 , and $\left(\hat{\hat{\beta}}_{3}, \infty\right)$ to Case 4 .
} 
2. It is profitable for the principal to adopt neither if $\theta=\theta_{3}$ nor if $\theta \in\left\{\theta_{1}, \theta_{2}\right\}$ :

$$
\begin{aligned}
\widehat{\pi}\left(1, \beta, \theta_{3}\right) & <\widehat{\pi}\left(0, \beta, \theta_{3}\right) \\
\frac{\rho_{1}}{\rho_{1}+\rho_{2}} \widehat{\pi}\left(1, \beta, \theta_{1}\right)+\frac{\rho_{2}}{\rho_{1}+\rho_{2}} \widehat{\pi}\left(1, \beta, \theta_{2}\right) & <\frac{\rho_{1}}{\rho_{1}+\rho_{2}} \widehat{\pi}\left(0, \beta, \theta_{1}\right)+\frac{\rho_{2}}{\rho_{1}+\rho_{2}} \widehat{\pi}\left(0, \beta, \theta_{2}\right)
\end{aligned}
$$

In this case, the principal's ex-ante expected profit (refer to (B16)) is:

$$
\pi^{*}(\beta)=\rho_{1} \widehat{\pi}\left(0, \beta, \theta_{1}\right)+\rho_{2} \widehat{\pi}\left(0, \beta, \theta_{2}\right)+\rho_{3} \widehat{\pi}\left(0, \beta, \theta_{3}\right)=\pi_{0}(\beta)
$$

From (B3), $\beta_{0}$ maximizes $\pi_{0}(\beta)$, i.e. $\pi_{0}\left(\beta_{0}\right) \geq \pi_{0}(\beta) \forall \beta$. But by Remark $2, \pi_{3}\left(\beta_{0}\right)>\pi_{0}\left(\beta_{0}\right)$ and hence $\pi^{*}\left(\beta_{0}\right)>\pi_{0}\left(\beta_{0}\right) \geq \pi_{0}(\beta)$ (where $\pi^{*}\left(\beta_{0}\right)$ is from (B17)). Hence the principal prefers the subgame with $\beta_{0}$ to any subgame that falls under this case.

3. It is profitable for the principal to adopt either if $\theta=\theta_{3}$ and or if $\theta \in\left\{\theta_{1}, \theta_{2}\right\}$ :

$$
\begin{aligned}
\widehat{\pi}\left(1, \beta, \theta_{3}\right) & \geq \widehat{\pi}\left(0, \beta, \theta_{3}\right) \\
\frac{\rho_{1}}{\rho_{1}+\rho_{2}} \widehat{\pi}\left(1, \beta, \theta_{1}\right)+\frac{\rho_{2}}{\rho_{1}+\rho_{2}} \widehat{\pi}\left(1, \beta, \theta_{2}\right) & \geq \frac{\rho_{1}}{\rho_{1}+\rho_{2}} \widehat{\pi}\left(0, \beta, \theta_{1}\right)+\frac{\rho_{2}}{\rho_{1}+\rho_{2}} \widehat{\pi}\left(0, \beta, \theta_{2}\right)
\end{aligned}
$$

In this case, the principal's ex-ante expected profit is:

$$
\pi^{*}(\beta)=\rho_{1} \pi_{1}(\beta)+\rho_{2} \pi_{2}(\beta)+\rho_{3} \pi_{3}(\beta)=\tilde{\pi}(\beta)
$$

where $\widetilde{\pi}(\cdot)$ is defined in (B5). Since $\widetilde{\beta}$ is the optimal choice if the principal bases her decision only on her priors and adopts (refer to $(\mathrm{B} 6))$, it must be the case that $\widetilde{\pi}(\widetilde{\beta}) \geq \widetilde{\pi}(\beta)$ for all $\beta$. But by condition $(\mathrm{B} 7 \mathrm{c}), \pi_{0}\left(\beta_{0}\right)>\widetilde{\pi}(\widetilde{\beta})$. As above, Remark 2 implies $\pi^{*}\left(\beta_{0}\right)>\pi_{0}\left(\beta_{0}\right)$ (where $\pi^{*}\left(\beta_{0}\right)$ is from $\left.(\mathrm{B} 17)\right)$. Hence $\pi^{*}\left(\beta_{0}\right)>\pi_{0}\left(\beta_{0}\right)>\widetilde{\pi}(\widetilde{\beta}) \geq \widetilde{\pi}(\beta) \forall \beta$; the principal prefers the subgame with $\beta_{0}$ to any subgame that falls under this case.

4. It is not profitable for the principal to adopt if $\theta=\theta_{3}$ but it is profitable if $\theta \in\left\{\theta_{1}, \theta_{2}\right\}:^{14}$

$$
\begin{aligned}
\widehat{\pi}\left(1, \beta, \theta_{3}\right) & <\widehat{\pi}\left(0, \beta, \theta_{3}\right) \\
\frac{\rho_{1}}{\rho_{1}+\rho_{2}} \widehat{\pi}\left(1, \beta, \theta_{1}\right)+\frac{\rho_{2}}{\rho_{1}+\rho_{2}} \widehat{\pi}\left(1, \beta, \theta_{2}\right) & \geq \frac{\rho_{1}}{\rho_{1}+\rho_{2}} \widehat{\pi}\left(0, \beta, \theta_{1}\right)+\frac{\rho_{2}}{\rho_{1}+\rho_{2}} \widehat{\pi}\left(0, \beta, \theta_{2}\right)
\end{aligned}
$$

In this case, the principal's ex-ante expected profit (refer to (B16)) is:

$$
\pi^{*}(\beta)=\rho_{1} \pi_{1}(\beta)+\rho_{2} \pi_{2}(\beta)+\rho_{3} \pi_{0}(\beta)
$$

Consider the function $\widetilde{\widetilde{\pi}}(\beta)=\rho_{1} \pi_{1}(\beta)+\rho_{2} \pi_{2}(\beta)+\rho_{3} \pi_{0}(\beta)$ defined over all possible values of $\beta$. (That is, $\widetilde{\widetilde{\pi}}(\cdot)$ and $\pi^{*}(\cdot)$ coincide for values of $\beta$ that yield Case 4 , but for values of $\beta$ that yield the other cases $\pi^{*}(\cdot) \geq \widetilde{\widetilde{\pi}}(\cdot)$ since $\pi^{*}(\cdot)$ reflects optimal adoption decisions.) Maximizing $\widetilde{\widetilde{\pi}}(\beta)$ over $\beta$ yields:

$$
\widetilde{\widetilde{\beta}}=\left(\frac{\rho_{1} s_{1}^{2}+\rho_{3} s_{0}^{2}}{\rho_{1} s_{1}^{2}+\rho_{2} s_{2}^{2}+\rho_{3} s_{0}^{2}}\right) \beta_{0}+\left(\frac{\rho_{2} s_{2}^{2}}{\rho_{1} s_{1}^{2}+\rho_{2} s_{2}^{2}+\rho_{3} s_{0}^{2}}\right) \beta_{2}
$$

Because $\widetilde{\widetilde{\beta}}$ maximizes $\widetilde{\widetilde{\pi}}(\beta)$ and $\widetilde{\widetilde{\pi}}(\cdot)$ is strictly convex, $\widetilde{\widetilde{\pi}}(\widetilde{\widetilde{\beta}})>\widetilde{\widetilde{\pi}}(\beta) \forall \beta \neq \widetilde{\widetilde{\beta}}$. Because $\widetilde{\widetilde{\beta}}$ is

\footnotetext{
${ }^{14}$ We include this case for completeness, although no informative equilibria will exist in this case as interests are completely misaligned.
} 
a weighted average of $\beta_{0}$ and $\beta_{2}$, we know $\widetilde{\widetilde{\beta}} \in\left(\beta_{0}, \beta_{2}\right)$. By $\operatorname{Remark} 2, \pi_{3}(\beta)>\pi_{0}(\beta)$ for all $\beta \in\left(\beta_{0}, \beta_{2}\right)$ and hence $\pi^{*}(\widetilde{\widetilde{\beta}})>\widetilde{\widetilde{\pi}}(\widetilde{\widetilde{\beta}})$ in this region. Since it is profitable to adopt $\theta_{3}$ for $\beta=\widetilde{\widetilde{\beta}}$, Case 1 or 3 must hold. By the argument in one or the other case, $\pi^{*}\left(\beta_{0}\right)>\widetilde{\widetilde{\pi}}(\widetilde{\widetilde{\beta}})$ (where $\pi^{*}\left(\beta_{0}\right)$ is from $(\mathrm{B} 17)$ ). Hence $\pi^{*}\left(\beta_{0}\right)>\widetilde{\widetilde{\pi}}(\widetilde{\widetilde{\beta}})>\widetilde{\widetilde{\pi}}(\beta)$ for values of $\beta$ for which this case holds. Once again, the principal prefers the subgame with $\beta_{0}$ to any subgame that falls under this case.

Considering the four cases together, we can conclude the maximum possible payoff to the principal for $\beta \neq \beta_{0}$ is less than $\pi^{*}\left(\beta_{0}\right)$ from (B17).

Intuitively, from Lemma 1 , types $\theta_{1}$ and $\theta_{2}$ can never be distinguished. Therefore, the highest possible payoff to the principal from any subgame equals the payoff that the principal would obtain if she observed whether $\theta=\theta_{3}$ or $\theta \in\left\{\theta_{1}, \theta_{2}\right\} .{ }^{15}$ It is possible to show that the payoff when $\beta=\beta_{0}$ is greater than the maximal payoffs in all other subgames.

Given Lemma 3, the principal does not have an incentive to deviate from $\beta^{*}=\beta_{0}$, her chosen wage in Proposition 1. By Lemma 2, the strategies outlined in Proposition 1 form a perfect Bayesian equilibrium of the subgame with $\beta^{*}=\beta_{0}$. Hence neither player has an incentive to deviate at any stage. This completes the proof of the existence of the equilibrium described in Proposition 1.

\section{B.2.2 Discussion}

Crawford and Sobel (1982) and others have argued that it is reasonable to assume that players coordinate on the most informative equilibrium in cheap-talk interactions. If one is willing to assume this, then the equilibrium described by Proposition 1 is unique. Recall that Lemma 1 implies that there are at most two possible equilibria in each subgame for each $\beta$. The restriction that we focus on the most informative equilibrium in each subgame implies that there is at most one equilibrium in each subgame. Lemma 3 implies that the principal prefers the subgame with $\beta=\beta_{0}$ to all other subgames. Hence the only equilibrium of the supergame is the one characterized by the Proposition-1 strategies on the equilibrium path.

One other result is worth highlighting. Lemma 1 implies that there does not exist an equilibrium in which the agent always truthfully reveals the technology type. That is, information about the technology is necessarily lost in some states of the world. Intuitively, if the agent were to reveal the technology type truthfully, then the principal would want to adopt type $\theta_{2}$ and not type $\theta_{1}$. But given this strategy of the principal, and the fact that the wage contract is fixed ex ante, the agent would be better off misreporting type $\theta_{2}$ to be type $\theta_{1}$.

\section{B.3 Conditional contracts}

Now suppose that in Stage 0 the principal can pay a transaction cost $G$ and gain access to a larger set of wage contracts - in particular to contracts that condition the piece rate on marginal cost, $c$. This larger set of possible contracts includes contracts that offer a per-sheet incentive to reduce waste of laminated rexine, as these can be interpreted as an increase in the piece rate conditional on using the lower-marginal-cost technology. ${ }^{16}$ The fixed cost $G$ can be interpreted as the cost of a commitment device to pay a piece rate above the one that would be paid for the existing technology

\footnotetext{
${ }^{15}$ This is a simple application of Blackwell's ordering, i.e. that the decision maker's payoff must be weakly higher with more information (see Blackwell (1953).)

${ }^{16}$ In our framework, the only way to reduce waste is to use the low-marginal-cost technology; exerting additional effort would raise output but not reduce waste per sheet.
} 
$\left(\beta_{0}\right)$, which otherwise the principal would not be able to credibly commit to. (We discuss other possible interpretations in Section 5.2.3 of the main text.)

The optimal contracts under the existing technology and types $\theta_{1}$ and $\theta_{3}$ are identical (since $c_{3}=c_{1}=c_{0}$ and hence $\beta_{3}=\beta_{1}=\beta_{0}$ ). The ability to condition on marginal cost matters only if the technology is type $\theta_{2}$. Allowing for conditioning, the principal can offer contracts of the form:

$$
\begin{array}{cc}
w(q)=\alpha+\left(\beta+\gamma_{2}\right) q & \text { if } c=c_{2} \\
w(q)=\alpha+\beta q & \text { if } c \neq c_{2}
\end{array}
$$

If $G$ is sufficiently small, then there exists an equilibrium in which the agent reports truthfully, in the sense that he encourages adoption of profitable technologies and discourages adoption of unprofitable ones. Again, for conciseness, the proposition only states the on-equilibrium-path strategies, but the proof below covers the entire strategy space.

Proposition 2. Under ( $\left.\mathrm{B}^{\mathrm{y}} \mathrm{a}\right)-(\mathrm{B} / \mathrm{c})$, if contracts conditioned on marginal cost are available at fixed cost $G$, then the following strategies are part of a perfect Bayesian equilibrium.

1. In Stages 0 and 1,

(a) For

$$
\rho_{2}>\frac{G}{\pi_{2}\left(\beta_{2}\right)-\pi_{0}\left(\beta_{0}\right)},
$$

the principal pays $G$ and offers wage contract $\left(\alpha^{* *}=0, \beta^{* *}=\frac{p-c_{0}}{2}, \gamma_{2}^{* *}=\frac{c_{0}-c_{2}}{2}\right)$.

(b) For $\rho_{2} \leq \frac{G}{\pi_{2}\left(\beta_{2}\right)-\pi_{0}\left(\beta_{0}\right)}$ the principal does not pay $G$ and offers wage contract $\left(\alpha^{* *}=0, \beta^{* *}=\frac{p-c_{0}}{2}\right)$.

2. In Stage 3,

(a) given $\left(\alpha^{* *}=0, \beta^{* *}=\frac{p-c_{0}}{2}, \gamma_{2}^{* *}=\frac{c_{0}-c_{2}}{2}\right)$, the agent:

$i$. says "technology is bad" if the technology is type $\theta_{1}$,

ii. says "technology is good" if the technology is type $\theta_{2}$ or $\theta_{3}$.

(b) given $\left(\alpha^{* *}=0, \beta^{* *}=\frac{p-c_{0}}{2}\right)$, the agent:

$i$. says "technology is bad" if the technology is type $\theta_{1}$ or $\theta_{2}$,

ii. says "technology is good" if the technology is type $\theta_{3}$.

3. In Stage 4, the principal:

(a) adopts if the agent says "technology is good",

(b) does not adopt if the agent says "technology is bad".

Intuitively, if the principal offers the conditional contract, the higher piece rate if $c=c_{2}$ is enough to induce the agent to prefer adoption if the technology is of type $\theta_{2} \cdot{ }^{17}$ Paying the transaction cost, $G$, will be in the interest of the principal if (B23) is satisfied, which is to say that the expected additional profit from adopting type $\theta_{2}$ (with the optimal piece rate for type $\theta_{2}$ ) is greater than the fixed cost of offering the new contract. In this case, the availability of the conditional contract solves the misinformation problem, in that type $\theta_{2}$ will be adopted in equilibrium. At the same time, if (B23) is not satisfied, for instance because the principal has a low prior, $\rho_{2}$, then there again exists the equilibrium of Proposition 1, in which type $\theta_{2}$ is not adopted.

\footnotetext{
${ }^{17}$ Note that, using the notation of $(\mathrm{B} 4), \beta^{* *}=\beta_{0}$ and $\beta^{* *}+\gamma_{2}^{* *}=\beta_{2}$, the optimal piece rate for type $\theta_{2}$ in the full-information case.
} 


\section{B.3.1 Proof of Proposition 2}

To prove this proposition, in Subsection B.3.1.1 we consider the subgame conditional on paying $G$ and offering $\beta^{* *}$ and $\gamma_{2}^{* *}$ and show first that the strategies in 2(a) and 3(a) in the proposition are part of a perfect Bayesian subgame equilibrium and second that the equilibrium replicates the payoff to the principal if the technology type were fully revealed to the principal at the beginning of Stage 1 (before setting the contract). This implies that, conditional on paying $G$, the principal cannot do better by choosing a subgame with a different $\beta$ and $\gamma_{2}$. In Subsection B.3.1.2, we consider the principal's decision about whether to pay $G$ and show that the strategies outlined in the proposition form an equilibrium of the full game.

\section{B.3.1.1 Subgame with $G$ paid, $\beta=\beta^{* *}, \gamma_{2}=\gamma_{2}^{* *}$}

As before, for types $\theta_{1}$ and $\theta_{3}, \frac{\partial}{\partial a} \widehat{U}\left(a, \beta, \theta_{1}\right)<0$ and $\frac{\partial}{\partial a} \widehat{U}\left(a, \beta, \theta_{3}\right)>0$ for all $a \in[0,1]$, since $s_{1}<s_{0}$ and $s_{3}>s_{0}$. (Refer to (B9).) For type $\theta_{2}$, expected utility is now given by:

$$
\widehat{U}\left(a, \beta, \gamma_{2}, \theta_{2}\right)=a\left(\frac{\left(\beta+\gamma_{2}\right)^{2} s_{2}^{2}}{2}\right)+(1-a)\left(\frac{\beta^{2} s_{0}^{2}}{2}\right)
$$

Here condition (B7a) implies that $\frac{\partial}{\partial a} \widehat{U}\left(a, \beta, \gamma_{2}, \theta_{2}\right)>0$ for $\beta=\beta^{* *}, \gamma_{2}=\gamma_{2}^{* *}$ and hence that agent type $\theta_{2}$ wants to encourage adoption. ${ }^{18}$ In this subgame, a result analogous to Lemma 1 holds. ${ }^{19}$

Lemma 4. In any equilibrium with $a_{\min }<a_{\max }$ the following statements are true:

$$
\begin{aligned}
& \sum_{m \in M_{a_{m i n}}} q\left(m \mid \theta_{1}\right)=1 \quad \sum_{m \in M_{a_{\max }}} q\left(m \mid \theta_{1}\right)=0 \quad \sum_{m \notin\left\{M_{a_{\min }} \cup M_{a_{\max }}\right\}} q\left(m \mid \theta_{1}\right)=0 \\
& \sum_{m \in M_{a_{m i n}}} q\left(m \mid \theta_{2}\right)=0 \quad \sum_{m \in M_{a_{\max }}} q\left(m \mid \theta_{2}\right)=1 \quad \sum_{m \notin\left\{M_{a_{m i n}} \cup M_{a_{m a x}}\right\}} q\left(m \mid \theta_{2}\right)=0 \\
& \sum_{m \in M_{a_{\min }}} q\left(m \mid \theta_{3}\right)=0 \quad \sum_{m \in M_{a_{\max }}} q\left(m \mid \theta_{3}\right)=1 \quad \sum_{m \notin\left\{M_{a_{\min }} \cup M_{a_{m a x}}\right\}} q\left(m \mid \theta_{3}\right)=0
\end{aligned}
$$

For $m \in M_{a_{\text {min }}}, p\left(\theta_{1} \mid m\right)=1, p\left(\theta_{2} \mid m\right)=0$, and $p\left(\theta_{3} \mid m\right)=0$. For $m \in M_{a_{\text {max }}}, p\left(\theta_{1} \mid m\right)=0$, $p\left(\theta_{2} \mid m\right)=\frac{\rho_{2}}{\rho_{2}+\rho_{3}}$, and $p\left(\theta_{3} \mid m\right)=\frac{\rho_{3}}{\rho_{2}+\rho_{3}}$.

In any equilibrium with $a_{\min }=a_{\max }$ (letting $a^{*} \equiv a_{\min }=a_{\max }$ ), the following statements are true:

$$
\sum_{m \in M_{a^{*}}} q\left(m \mid \theta_{i}\right)=1 \forall \theta_{i}, \quad \sum_{m \notin M_{a^{*}}} q\left(m \mid \theta_{i}\right)=0 \forall \theta_{i}
$$

For $m \in M_{a^{*}}, p\left(\theta_{1} \mid m\right)=\rho_{1}, p\left(\theta_{2} \mid m\right)=\rho_{2}$, and $p\left(\theta_{3} \mid m\right)=\rho_{3}$.

Proof. Given that $\frac{\partial}{\partial a} \widehat{U}\left(a, \beta, \theta_{1}\right)<0, \frac{\partial}{\partial a} \widehat{U}\left(a, \beta, \theta_{2}\right)>0$ and $\frac{\partial}{\partial a} \widehat{U}\left(a, \beta, \theta_{3}\right)>0$ for all $a \in[0,1]$, if $a_{\min } \neq a_{\max }$ then in order for (B12) to be satisfied it must be the case that an agent of type $\theta_{1}$ chooses messages that induce the lowest possible probability of adoption, $a_{m i n}$, and agents of type $\theta_{2}$ or $\theta_{3}$ choose a message that induces the highest possible probability of adoption, $a_{\max }$. Given these reporting rules, the updating of the principal's beliefs follow by Bayes' rule. If $a_{\min }=a_{\max }$

\footnotetext{
${ }^{18}$ To see this, note that condition (B7a) implies $\pi_{2}\left(\beta_{2}\right)>\pi_{0}\left(\beta_{0}\right)$, since $\pi_{2}(\beta)$ is increasing for $\beta \in\left[\beta_{0}, \beta_{2}\right)$. Using (B3) and (B4), we have that $\frac{\left(\left(\beta^{* *}+\gamma_{2}^{* *}\right) s_{2}\right)^{2}}{2}>\frac{\left(\beta^{* *} s_{0}\right)^{2}}{2}$, which in turn implies $\frac{\partial}{\partial a} \widehat{U}\left(a, \beta, \gamma_{2}, \theta_{2}\right)>0$.

${ }^{19}$ This lemma holds in any subgame in which $\gamma_{2}>\beta\left(\frac{s_{0}-s_{2}}{s_{2}}\right)$ and hence $\frac{\partial}{\partial a} \widehat{U}\left(a, \beta, \gamma_{2}, \theta_{2}\right)>0$.
} 
then trivially all messages sent by the agent induce the same action and hence are essentially equivalent $\left(M_{a_{\min }}=M_{a_{\max }}=M_{a^{*}}\right)$. Given this, the principal does not update.

As in Lemma 1, we have shown that there exist at most two equilibria in this subgame. We can also show existence of the informative subgame equilibrium.

Lemma 5. If $G$ is paid, $\beta^{* *}=\frac{p-c_{0}}{2}$ and $\gamma_{2}^{* *}=\frac{c_{0}-c_{2}}{2}$, there exists a perfect Bayesian subgame equilibrium with the strategies outlined in Proposition 2.

Proof. In the subgame equilibrium outlined by Proposition 2 (after paying $G$ ), the agent's reporting rules are given by $(\mathrm{B} 25 \mathrm{a})-(\mathrm{B} 25 \mathrm{c})$, where $a_{\min }=0, a_{\max }=1$, and the principal's action rule is $a(m)=0 \forall m \in M_{0}, a(m)=1 \forall m \in M_{1}$.

To prove that this is a subgame equilibrium, it suffices to show that neither agent nor principal wants to deviate. Since $\frac{\partial}{\partial a} \widehat{U}\left(a, \beta, \theta_{1}\right)<0, \frac{\partial}{\partial a} \widehat{U}\left(a, \beta, \theta_{2}\right)>0$ and $\frac{\partial}{\partial a} \widehat{U}\left(a, \beta, \theta_{3}\right)>0$ for all $a \in[0,1]$, the agent has no incentive to deviate. Now consider the principal's decision. Given Lemma 4, if the agent says $m \in M_{0}$ ("technology is bad"), the condition for the principal not to deviate is:

$$
\pi_{0}\left(\beta^{* *}\right)>\pi_{1}\left(\beta^{* *}\right)
$$

which is satisfied for $\beta=\beta^{* *}=\beta_{0}$ by Remark 3. If the agent says $m \in M_{1}$ ("technology is good"), the condition for the principal not to deviate is:

$$
\pi_{0}\left(\beta_{0}\right) \leq\left(\frac{\rho_{2}}{\rho_{2}+\rho_{3}}\right) \pi_{2}\left(\beta_{2}\right)+\left(\frac{\rho_{3}}{\rho_{2}+\rho_{3}}\right) \pi_{3}\left(\beta_{0}\right)
$$

since $\beta^{* *}=\beta_{0}$ and $\beta^{* *}+\gamma_{2}^{* *}=\beta_{2}$. Since $\pi_{2}(\beta)$ is increasing for $\beta \in\left[\beta_{0}, \beta_{2}\right)$, condition (B7a) implies $\pi_{0}\left(\beta_{0}\right)<\pi_{2}\left(\beta_{2}\right)$ as noted in Remark 1. Similarly $\pi_{0}\left(\beta_{0}\right)<\pi_{3}\left(\beta_{0}\right)$ follows from condition (B7b) as noted in Remark 2. Hence the right-hand-side of (B27) is a weighted average of two quantities greater than $\pi_{0}\left(\beta_{0}\right)$ and $(\mathrm{B} 27)$ is satisfied.

In this subgame, the principal's ex-ante expected profit (refer to (B16)) is:

$$
\begin{aligned}
\pi^{* *}\left(\beta^{* *}, \gamma_{2}^{* *}\right) & =\rho_{1} \widehat{\pi}\left(0, \beta_{0}, \theta_{1}\right)+\rho_{2} \widehat{\pi}\left(1, \beta_{2}, \theta_{2}\right)+\rho_{3} \widehat{\pi}\left(1, \beta_{0}, \theta_{3}\right)-G \\
& =\rho_{1} \pi_{0}\left(\beta_{0}\right)+\rho_{2} \pi_{2}\left(\beta_{2}\right)+\rho_{3} \pi_{3}\left(\beta_{0}\right)-G
\end{aligned}
$$

Compare this payoff to what would obtain in the conditional contracts case if the technology were fully revealed to the principal at the beginning of Stage 1. Recalling (B4), the principal would offer $\beta_{0}$ under the existing technology and types $\theta_{1}$ and $\theta_{3}$ and $\beta_{2}$ under type $\theta_{2}$. She would adopt types $\theta_{2}$ and $\theta_{3}$, and not adopt type $\theta_{1} \cdot{ }^{20}$ Her ex-ante expected profit would be equal to (B28). That is, when $G$ is paid, the conditional contract with $\beta^{* *}$ and $\gamma_{2}^{* *}$ exactly replicates the payoff to the principal if she observed the technology type herself at the beginning of Stage 1 . The insight of Blackwell (1953), mentioned above, is that the principal cannot do better than she would do with full information. Hence conditional on paying $G$, no subgame can offer the principal a better payoff than the one she receives from offering $\left(\beta^{* *}, \gamma_{2}^{* *}\right)$. Conditional on paying $G$, the principal has no incentive to deviate to offer a different $\beta$ or $\gamma_{2}$.

\footnotetext{
${ }^{20}$ To see this, note that $\pi_{0}\left(\beta_{0}\right)>\pi_{1}\left(\beta_{1}\right)$ and $\pi_{0}\left(\beta_{0}\right)<\pi_{3}\left(\beta_{0}\right)$ from Remarks 2-3, and $\pi_{0}\left(\beta_{0}\right)<\pi_{2}\left(\beta_{2}\right)$ from Remark 1 (and the fact that $\pi_{2}\left(\beta_{2}\right) \geq \pi_{2}\left(\beta_{0}\right)$ since $\beta_{2}$ is the optimal wage under type $\theta_{2}$ ).
} 


\section{B.3.1.2 Principal's choice whether to pay $G$}

We now consider whether the principal has an incentive to deviate from the strategies outlined in Proposition 2 at Stage 0, when choosing whether to pay $G$. Suppose $\rho_{2}>\frac{G}{\pi_{2}\left(\beta_{2}\right)-\pi_{0}\left(\beta_{0}\right)}$ (i.e. that (B23) is satisfied). If the principal pays $G$, her payoff is given by $\pi^{* *}\left(\beta^{* *}, \gamma_{2}^{* *}\right)$ in (B28) above. If she were to deviate and not pay $G$, the resulting subgame would identical to the interaction analyzed in Proposition 1. In this case, the maximal payoff she could obtain would be $\pi^{*}\left(\beta_{0}\right)$ from (B17). If (B23) holds, then $\pi^{* *}\left(\beta^{* *}, \gamma_{2}^{* *}\right)>\pi^{*}\left(\beta_{0}\right)$ and she does not have an incentive to deviate to receive the maximal payoff from not paying $G$. If this is true for the maximal payoff from deviating, it is also true for all other payoffs from deviating.

Similarly, suppose $\rho_{2} \leq \frac{G}{\pi_{2}\left(\beta_{2}\right)-\pi_{0}\left(\beta_{0}\right)}$ (i.e. that (B23) is not satisfied). ${ }^{21}$ If the principal does not pay $G$ and offers $\beta=\beta_{0}=\frac{p-c_{0}}{2}$, her payoff is given by $\pi^{*}\left(\beta_{0}\right)$ from (B17). If she were to deviate and pay $G$, the maximal payoff she could obtain is $\pi^{* *}\left(\beta^{* *}, \gamma_{2}^{* *}\right)$ in (B28). If (B23) holds, then $\pi^{* *}\left(\beta^{* *}, \gamma_{2}^{* *}\right)<\pi^{*}\left(\beta_{0}\right)$ and she does not have an incentive to deviate to receive the maximal payoff from paying $G$. If this is true for the maximal payoff from deviating, it is also true for all other payoffs from deviating.

We have already shown that neither player has an incentive to deviate in the resulting subgames. Hence the strategies described in Proposition 2 form a perfect Bayesian equilibrium.

\section{B.3.2 Uniqueness of the equilibrium in Proposition 2}

As with Proposition 1, if we are willing to assume that players coordinate on the most informative equilibrium in cheap-talk interactions, the equilibrium described by Proposition 2 is unique. Recall that in the case where $\rho_{2} \leq \frac{G}{\pi_{2}\left(\beta_{2}\right)-\pi_{0}\left(\beta_{0}\right)}$, the equilibrium is identical to that in Proposition 1 which was unique if players coordinated on the most informative equilibrium of each subgame. Thus, to prove that the equilibrium in Proposition 2 is unique, we only need to show that the equilibrium is unique in the case where $\rho_{2}>\frac{G}{\pi_{2}\left(\beta_{2}\right)-\pi_{0}\left(\beta_{0}\right)}$. We do this in two steps. First, Lemma 6 shows that in any subgame conditional on a wage contract, there are at most two equilibria and these can be strictly ordered in terms of which is most informative. Second, Lemma 7 shows that the principal's ex-ante expected profits under the contract $\left(\beta^{* *}, \gamma_{2}^{* *}\right)$ are strictly greater than under any other possible wage contract, and so the principal prefers that subgame to any other. Hence, the only equilibrium of the supergame conditional on paying $G$ is the one characterized by the Proposition- 2 strategies on the equilibrium path.

Lemma 6. In any subgame conditional on a wage contract, there are at most two equilibria, one of which is strictly more informative that the other.

Proof. Under conditional contracts, the principal can offer contracts of the form:

$$
\begin{array}{cc}
w(q)=\alpha+\left(\beta+\gamma_{1}\right) q & \text { if } c=c_{1} \\
w(q)=\alpha+\left(\beta+\gamma_{2}\right) q & \text { if } c=c_{2} \\
w(q)=\alpha+\left(\beta+\gamma_{3}\right) q & \text { if } c=c_{3} \\
w(q)=\alpha+\beta q & \text { if } c=c_{0}
\end{array}
$$

As in Proposition 1, $\alpha>0$ is costly and does not induce effort and so the principal will always set $\alpha=0$. We denote a set of piece-rate contracts by $\left(\beta, \gamma_{1}, \gamma_{2}, \gamma_{3}\right)$. (Note that the contract in

\footnotetext{
${ }^{21}$ We implicitly assume that the principal prefers the simpler option of not paying $G$ if $G=\rho_{2}\left(\pi_{2}\left(\beta_{2}\right)-\pi_{0}\left(\beta_{0}\right)\right)$.
} 
Proposition 2 corresponds to $\left(\frac{p-c_{0}}{2}, 0, \frac{c_{0}-c_{2}}{2}, 0\right)$ in this notation.) Define the expected utility of the agent of type $\theta$, prior to the adoption decision of the principal:

$$
\widehat{\widehat{U}}\left(a, \beta, \gamma_{i}, \theta_{i}\right)=a U\left(\beta+\gamma_{i}, \theta_{i}\right)+(1-a) U\left(\beta, \theta_{0}\right)
$$

where $U(\cdot, \cdot)$ is as defined in (B2) and where $\frac{\partial}{\partial a} \widehat{\widehat{U}}\left(a, \beta, \gamma_{i}, \theta_{i}\right)=U\left(\beta+\gamma_{i}, \theta_{i}\right)-U\left(\beta, \theta_{0}\right)$ is independent of $a$. We assume a tie breaking rule where the principal and agent prefer the status quo technology if $U\left(\beta+\gamma_{i}, \theta_{i}\right)=U\left(\beta, \theta_{0}\right)$.

Define $\widehat{\widehat{\pi}}\left(a, \beta, \gamma_{i}, \theta_{i}\right)$ as the expected profit of the principal prior to the adoption decision, conditional on $\theta_{i}$ :

$$
\widehat{\widehat{\pi}}\left(a, \beta, \gamma_{i}, \theta_{i}\right)=a \pi_{i}\left(\beta+\gamma_{i}\right)+(1-a) \pi_{0}(\beta)
$$

where $\pi_{i}(\cdot)$ is as defined in in (B3). As before, the principal's posterior beliefs after receiving message $m$, by Bayes' rule, are given by (B11). An equilibrium in the cheap-talk subgame is a family of reporting rules $q(m \mid \theta)$ for the agent (sender) and an action rule $a(m)$ for the principal (receiver) such that the following conditions hold:

1. If $q\left(m^{*} \mid \theta\right)>0$ then

$$
m^{*}=\underset{m \in M}{\arg \max } \widehat{\widehat{U}}\left(a(m), \beta, \gamma_{i}, \theta\right)
$$

2. For each $m$,

$$
a(m)=\underset{a \in[0,1]}{\arg \max } \sum_{\theta \in \Theta} \widehat{\widehat{\pi}}\left(a, \beta, \gamma_{i}, \theta\right) p(\theta \mid m)
$$

That is, the rule of each player must be a best response to the rule of the other player.

For there to be more than one equilibrium, it must be the case that $a_{\min }<a_{\max }$. In any equilibrium with $a_{\min }<a_{\max }$, the following statements are true:

$$
\begin{aligned}
& \sum_{m \in M_{a_{m i n}}} q\left(m \mid \theta_{1}\right)=1 \sum_{m \in M_{a_{m a x}}} q\left(m \mid \theta_{1}\right)=0 \quad \sum_{m \notin\left\{M_{a_{m i n}} \cup M_{a_{m a x}}\right\}} q\left(m \mid \theta_{1}\right)=0 \text { if } \frac{\partial}{\partial a} \widehat{\widehat{U}}\left(a, \beta, \gamma_{1}, \theta_{1}\right) \leq 0 \\
& \sum_{m \in M_{a_{m i n}}} q\left(m \mid \theta_{1}\right)=0 \sum_{m \in M_{a_{m a x}}} q\left(m \mid \theta_{1}\right)=1 \sum_{m \notin\left\{M_{a_{m i n}} \cup M_{a_{m a x}}\right\}} q\left(m \mid \theta_{1}\right)=0 \text { if } \frac{\partial}{\partial a} \widehat{\widehat{U}}\left(a, \beta, \gamma_{1}, \theta_{1}\right)>0 \\
& \sum_{m \in M_{a_{m i n}}} q\left(m \mid \theta_{2}\right)=1 \sum_{m \in M_{a_{m a x}}} q\left(m \mid \theta_{2}\right)=0 \quad \sum_{m \notin\left\{M_{a_{m i n}} \cup M_{a_{m a x}}\right\}} q\left(m \mid \theta_{2}\right)=0 \text { if } \frac{\partial}{\partial a} \widehat{\widehat{U}}\left(a, \beta, \gamma_{2}, \theta_{2}\right) \leq 0 \\
& \sum_{m \in M_{a_{m i n}}} q\left(m \mid \theta_{2}\right)=0 \sum_{m \in M_{a_{m a x}}} q\left(m \mid \theta_{2}\right)=1 \sum_{m \notin\left\{M_{a_{m i n}} \cup M_{a_{m a x}}\right\}} q\left(m \mid \theta_{2}\right)=0 \text { if } \frac{\partial}{\partial a} \widehat{\widehat{U}}\left(a, \beta, \gamma_{2}, \theta_{2}\right)>0
\end{aligned}
$$




$$
\begin{aligned}
\sum_{m \in M_{a_{m i n}}} q\left(m \mid \theta_{3}\right) & =1 \sum_{m \in M_{a_{m a x}}} q\left(m \mid \theta_{3}\right)=0 & \sum_{m \notin\left\{M_{a_{m i n}} \cup M_{a_{m a x}}\right\}} q\left(m \mid \theta_{3}\right)=0 \text { if } \frac{\partial}{\partial a} \widehat{\widehat{U}}\left(a, \beta, \gamma_{3}, \theta_{3}\right) \leq 0 \\
\sum_{m \in M_{a_{\text {min }}}} q\left(m \mid \theta_{3}\right)=0 & \sum_{m \in M_{a_{\text {max }}}} q\left(m \mid \theta_{3}\right)=1 & \sum_{m \notin\left\{M_{a_{\text {min }}} \cup M_{a_{\text {max }}}\right\}} q\left(m \mid \theta_{3}\right)=0 \text { if } \frac{\partial}{\partial a} \widehat{\widehat{U}}\left(a, \beta, \gamma_{3}, \theta_{3}\right)>0
\end{aligned}
$$

Given the signs of $\frac{\partial}{\partial a} \widehat{\widehat{U}}\left(a, \beta, \gamma_{1}, \theta_{1}, \frac{\partial}{\partial a} \widehat{\widehat{U}}\left(a, \beta, \gamma_{2}, \theta_{2}\right)\right.$ and $\frac{\partial}{\partial a} \widehat{\widehat{U}}\left(a, \beta, \gamma_{3}, \theta_{3}\right)$ for all $a \in[0,1]$, if $a_{\min } \neq a_{\max }$ then in order for (B32) to be satisfied it must be the case that an agent of type $\theta_{i}$ chooses messages that induce the lowest possible probability of adoption, $a_{\text {min }}$, if $\frac{\partial}{\partial a} \widehat{\widehat{U}}\left(a, \beta, \gamma_{i}, \theta_{i}\right) \leq$ 0 , and chooses messages that induce the highest possible probability of adoption, $a_{\text {max }}$, if $\frac{\partial}{\partial a} \widehat{\widehat{U}}\left(a, \beta, \gamma_{i}, \theta_{i}\right)>$ 0 . For $a_{\min }<a_{\max }$, both messages must be used. Given these reporting rules, the updating of the principal's beliefs follow by Bayes' rule.

There are eight possible cases to consider:

1. If $\frac{\partial}{\partial a} \widehat{\widehat{U}}\left(a, \beta, \gamma_{1}, \theta_{1}\right) \leq 0, \frac{\partial}{\partial a} \widehat{\widehat{U}}\left(a, \beta, \gamma_{2}, \theta_{2}\right) \leq 0, \frac{\partial}{\partial a} \widehat{\widehat{U}}\left(a, \beta, \gamma_{3}, \theta_{3}\right) \leq 0$ : For $m \in M_{a_{m i n}}, p\left(\theta_{1} \mid m\right)=$ $\rho_{1}, p\left(\theta_{2} \mid m\right)=\rho_{2}$, and $p\left(\theta_{3} \mid m\right)=\rho_{3}$. For $m \in M_{a_{\max }}, p\left(\theta_{1} \mid m\right)=0, p\left(\theta_{2} \mid m\right)=0$, and $p\left(\theta_{3} \mid m\right)=0$. As only $a_{\min }$ is used there is no equilibrium with $a_{\min }<a_{\max }$. There is a single equilibrium with $a_{\min }=a_{\max }$.

2. If $\frac{\partial}{\partial a} \widehat{\widehat{U}}\left(a, \beta, \gamma_{1}, \theta_{1}\right) \leq 0, \frac{\partial}{\partial a} \widehat{\widehat{U}}\left(a, \beta, \gamma_{2}, \theta_{2}\right) \leq 0, \frac{\partial}{\partial a} \widehat{\widehat{U}}\left(a, \beta, \gamma_{3}, \theta_{3}\right)>0$ : For $m \in M_{a_{\text {min }}}, p\left(\theta_{1} \mid m\right)=$ $\frac{\rho_{1}}{\rho_{1}+\rho_{2}}, p\left(\theta_{2} \mid m\right)=\frac{\rho_{2}}{\rho_{1}+\rho_{2}}$, and $p\left(\theta_{3} \mid m\right)=0$. For $m \in M_{a_{\max }}, p\left(\theta_{1} \mid m\right)=0, p\left(\theta_{2} \mid m\right)=0$, and $p\left(\theta_{3} \mid m\right)=1$. As both $a_{\min }$ and $a_{\max }$ are used, there are at most two equilibria, an informative one with $a_{\min }<a_{\max }$ and potentially a babbling one with $a_{\min }=a_{\max }$.

3. If $\frac{\partial}{\partial a} \widehat{\widehat{U}}\left(a, \beta, \gamma_{1}, \theta_{1}\right) \leq 0, \frac{\partial}{\partial a} \widehat{\widehat{U}}\left(a, \beta, \gamma_{2}, \theta_{2}\right)>0, \frac{\partial}{\partial a} \widehat{\widehat{U}}\left(a, \beta, \gamma_{3}, \theta_{3}\right) \leq 0$ : For $m \in M_{a_{m i n}}, p\left(\theta_{1} \mid m\right)=$ $\frac{\rho_{1}}{\rho_{1}+\rho_{3}}, p\left(\theta_{2} \mid m\right)=0$, and $p\left(\theta_{3} \mid m\right)=\frac{\rho_{3}}{\rho_{1}+\rho_{3}}$. For $m \in M_{a_{\max }}, p\left(\theta_{1} \mid m\right)=0, p\left(\theta_{2} \mid m\right)=1$, and $p\left(\theta_{3} \mid m\right)=0$. As both $a_{\min }$ and $a_{\max }$ are used, there are at most two equilibria, an informative one with $a_{\min }<a_{\max }$ and potentially a babbling one with $a_{\min }=a_{\max }$.

4. If $\frac{\partial}{\partial a} \widehat{\widehat{U}}\left(a, \beta, \gamma_{1}, \theta_{1}\right) \leq 0, \frac{\partial}{\partial a} \widehat{\widehat{U}}\left(a, \beta, \gamma_{2}, \theta_{2}\right)>0, \frac{\partial}{\partial a} \widehat{\widehat{U}}\left(a, \beta, \gamma_{3}, \theta_{3}\right)>0$ : For $m \in M_{a_{m i n}}, p\left(\theta_{1} \mid m\right)=$ $1, p\left(\theta_{2} \mid m\right)=0$, and $p\left(\theta_{3} \mid m\right)=0$. For $m \in M_{a_{\text {max }}}, p\left(\theta_{1} \mid m\right)=0, p\left(\theta_{2} \mid m\right)=\frac{\rho_{2}}{\rho_{2}+\rho_{3}}$, and $p\left(\theta_{3} \mid m\right)=\frac{\rho_{3}}{\rho_{2}+\rho_{3}}$. As both $a_{\min }$ and $a_{\max }$ are used, there are at most two equilibria, an informative one with $a_{\min }<a_{\max }$ and potentially a babbling one with $a_{\min }=a_{\max }$.

5. If $\frac{\partial}{\partial a} \widehat{\widehat{U}}\left(a, \beta, \gamma_{1}, \theta_{1}\right)>, \frac{\partial}{\partial a} \widehat{\widehat{U}}\left(a, \beta, \gamma_{2}, \theta_{2}\right)>0, \frac{\partial}{\partial a} \widehat{\widehat{U}}\left(a, \beta, \gamma_{3}, \theta_{3}\right)>0$ : For $m \in M_{a_{m i n}}, p\left(\theta_{1} \mid m\right)=$ $0, p\left(\theta_{2} \mid m\right)=0$, and $p\left(\theta_{3} \mid m\right)=0$. For $m \in M_{a_{\text {max }}}, p\left(\theta_{1} \mid m\right)=\rho_{1}, p\left(\theta_{2} \mid m\right)=\rho_{2}$, and $p\left(\theta_{3} \mid m\right)=\rho_{3}$. As only $a_{\min }$ is used there is no equilibrium with $a_{\min }<a_{\max }$. There is a single equilibrium with $a_{\min }=a_{\max }$.

6. If $\frac{\partial}{\partial a} \widehat{\widehat{U}}\left(a, \beta, \gamma_{1}, \theta_{1}\right)>0, \frac{\partial}{\partial a} \widehat{\widehat{U}}\left(a, \beta, \gamma_{2}, \theta_{2}\right) \leq 0, \frac{\partial}{\partial a} \widehat{\widehat{U}}\left(a, \beta, \gamma_{3}, \theta_{3}\right) \leq 0$ : For $m \in M_{a_{\text {min }}}, p\left(\theta_{1} \mid m\right)=$ $0, p\left(\theta_{2} \mid m\right)=\frac{\rho_{2}}{\rho_{2}+\rho_{3}}$, and $p\left(\theta_{3} \mid m\right)=\frac{\rho_{3}}{\rho_{2}+\rho_{3}}$. For $m \in M_{a_{\max }}, p\left(\theta_{1} \mid m\right)=1, p\left(\theta_{2} \mid m\right)=0$, and $p\left(\theta_{3} \mid m\right)=0$. As both $a_{\min }$ and $a_{\max }$ are used, there are at most two equilibria, an informative one with $a_{\min }<a_{\max }$ and potentially a babbling one with $a_{\min }=a_{\max }$. 
7. If $\frac{\partial}{\partial a} \widehat{\widehat{U}}\left(a, \beta, \gamma_{1}, \theta_{1}\right)>0, \frac{\partial}{\partial a} \widehat{\widehat{U}}\left(a, \beta, \gamma_{2}, \theta_{2}\right) \leq 0, \frac{\partial}{\partial a} \widehat{\widehat{U}}\left(a, \beta, \gamma_{3}, \theta_{3}\right)>0$ : For $m \in M_{a_{m i n}}, p\left(\theta_{1} \mid m\right)=$ $0, p\left(\theta_{2} \mid m\right)=1$, and $p\left(\theta_{3} \mid m\right)=0$. For $m \in M_{a_{\text {max }}}, p\left(\theta_{1} \mid m\right)=\frac{\rho_{1}}{\rho_{1}+\rho_{3}}, p\left(\theta_{2} \mid m\right)=0$, and $p\left(\theta_{3} \mid m\right)=\frac{\rho_{3}}{\rho_{1}+\rho_{3}}$. As both $a_{\min }$ and $a_{\max }$ are used, there are at most two equilibria, an informative one with $a_{\min }<a_{\max }$ and potentially a babbling one with $a_{\min }=a_{\max }$.

8. If $\frac{\partial}{\partial a} \widehat{\widehat{U}}\left(a, \beta, \gamma_{1}, \theta_{1}\right)>0, \frac{\partial}{\partial a} \widehat{\widehat{U}}\left(a, \beta, \gamma_{2}, \theta_{2}\right)>0, \frac{\partial}{\partial a} \widehat{\widehat{U}}\left(a, \beta, \gamma_{3}, \theta_{3}\right) \leq 0$ : For $m \in M_{a_{m i n}}, p\left(\theta_{1} \mid m\right)=$ $0, p\left(\theta_{2} \mid m\right)=0$, and $p\left(\theta_{3} \mid m\right)=1$. For $m \in M_{a_{\text {max }}}, p\left(\theta_{1} \mid m\right)=\frac{\rho_{1}}{\rho_{1}+\rho_{2}}, p\left(\theta_{2} \mid m\right)=\frac{\rho_{2}}{\rho_{1}+\rho_{2}}$, and $p\left(\theta_{3} \mid m\right)=0$. As both $a_{\min }$ and $a_{\max }$ are used, there are at most two equilibria, an informative one with $a_{\min }<a_{\max }$ and potentially a babbling one with $a_{\min }=a_{\max }$.

Given $\left(\beta, \gamma_{1}, \gamma_{2}, \gamma_{3}\right)$, in cases 2-4 and 6-8 there can exist at most two equilibria in each subgame: one more-informative equilibrium in which $a_{\min } \neq a_{\max }$ and two agent types are indistinguishable from each other but are distinguishable from the third type; and one less-informative equilibrium in which $a_{\min }=a_{\max }$ and the principal ignores the message from the agent (a "babbling" type). In cases 1 and 5 , only a single equilibrium exists in which $a_{\min }=a_{\max }$ and the principal ignores the message from the agent.

Lemma 7. The principal's ex-ante expected profits under the contract $\left(\beta^{* *}, \gamma_{2}^{* *}\right)-\left(\beta^{* *}, 0, \gamma_{2}^{* *}, 0\right)$ using the notation of Lemma 6 - are strictly greater than under any other possible wage contract.

Proof. From (B4), the maximal ex-ante expected profits are equal to:

$$
\begin{aligned}
\pi^{\max } & =\rho_{1} \max \left(\pi_{0}\left(\beta_{0}\right), \pi_{1}\left(\beta_{1}\right)\right)+\rho_{2} \max \left(\pi_{0}\left(\beta_{0}\right), \pi_{2}\left(\beta_{2}\right)\right)+\rho_{3} \max \left(\pi_{0}\left(\beta_{0}\right), \pi_{3}\left(\beta_{3}\right)\right)-G \\
& =\rho_{1} \pi_{0}\left(\beta_{0}\right)+\rho_{2} \pi_{2}\left(\beta_{2}\right)+\rho_{3} \pi_{3}\left(\beta_{3}\right)-G
\end{aligned}
$$

where the second line follows from the fact $\pi_{0}\left(\beta_{0}\right)>\pi_{1}\left(\beta_{1}\right)$ and $\pi_{0}\left(\beta_{0}\right)<\pi_{3}\left(\beta_{0}\right)$ from Remarks 2-3 (and the fact that $\beta_{0}=\beta_{3}$ since $c_{0}=c_{3}$ ), and $\pi_{0}\left(\beta_{0}\right)<\pi_{2}\left(\beta_{2}\right)$ from Remark 1 (and the fact that $\pi_{2}\left(\beta_{2}\right) \geq \pi_{2}\left(\beta_{0}\right)$ since $\beta_{2}$ is the optimal wage under type $\left.\theta_{2}\right)$. This is the expected profit both under full information and under the contract $\left(\beta^{* *}, 0, \gamma_{2}^{* *}, 0\right)$. From Blackwell (1953), we know that the principal cannot do better than this.

It remains to show that other wage contracts and/or adoption patterns cannot provide equally large ex-ante expected profits. First, if the principal pays optimal piece rates, $\beta_{i}$ for type $\theta_{i}$, profits will be strictly smaller under any adoption patterns other than adopt $\theta_{2}$ and $\theta_{3}$, do not adopt $\theta_{1}: \pi_{0}\left(\beta_{0}\right)>\pi_{1}\left(\beta_{1}\right), \pi_{0}\left(\beta_{0}\right)<\pi_{2}\left(\beta_{2}\right)$, and $\pi_{0}\left(\beta_{0}\right)<\pi_{3}\left(\beta_{3}\right)$ are all strict inequalities. Second, profits at optimal piece rates, $\pi_{i}\left(\beta_{i}\right)$ for type $\theta_{i}$, are also strictly higher under piece rate $\beta_{i}$ than under any other piece rate: $\pi_{i}(\beta)$ is twice continuously differentiable and strictly concave as $\frac{\partial^{2} \pi_{i}(\beta)}{\partial \beta^{2}}=-2 s_{i}^{2}<0$; hence, the stationary point $\beta_{i}$ (the value of $\beta$ for which $\frac{\partial \pi_{i}(\beta)}{\partial \beta}=0$ ) is a global maximizer and any deviations from these optimal piece-rates will strictly reduce profits.

\section{B.4 Theoretical prediction for incentive intervention}

Here we prove that a lump-sum payment offered by a third-party experimenter conditional on the technology being revealed to be type $\theta_{2}$, if sufficiently large, can induce the agent to reveal truthfully and lead to adoption of the type $\theta_{2}$ technology. Suppose that the players have coordinated on the equilibrium described in Proposition 1 (or, equivalently, on the equilibrium in Proposition 2 where $G$ is large relative to the expected benefits of adopting type $\theta_{2}$ and hence (B23) is not 
satisfied and the conditional contracts are not offered.) In Stage 1, the principal offers wage contract $\left(\alpha^{*}=0, \beta^{*}=\frac{p-c_{0}}{2}\right)$. Now suppose that in Stage 2 a third-party experimenter, without forewarning, offers a conditional lump-sum payment, $L$, conditional on the marginal cost being $c_{2}$. Suppose that players place zero prior on this event in Stages 0 and 1. For the subgame that follows this intervention, we have the following.

Proposition 3. In the subgame described above, if

$$
L>\frac{\left(p-c_{0}\right)^{2}\left(s_{0}^{2}-s_{2}^{2}\right)}{8}
$$

then the following strategies are part of a perfect Bayesian subgame equilibrium.

1. In Stage 3, the agent:

(a) says "technology is bad" if the technology is type $\theta_{1}$,

(b) says "technology is good" if the technology is type $\theta_{2}$ or $\theta_{3}$.

2. In Stage 4, the principal:

(a) adopts if the agent says "technology is good",

(b) does not adopt if the agent says "technology is bad".

Proof. It again suffices to show that there is no profitable deviation for either principal or agent. In the subgame equilibrium outlined by Proposition 3, the agent's reporting rules are given by (B25a)-(B25c), where $a_{\min }=0, a_{\max }=1$, and the principal's action rule is $a(m)=0 \forall m \in M_{0}$, $a(m)=1 \forall m \in M_{1}$. That the agent does not want to deviate if he is of type $\theta_{1}$ or $\theta_{3}$ follows from the fact (from (B9)) that $\frac{\partial}{\partial a} \widehat{U}\left(a, \beta, \theta_{1}\right)<0$ and $\frac{\partial}{\partial a} \widehat{U}\left(a, \beta, \theta_{3}\right)>0$ for all $a \in[0,1]$. That the agent does not want to deviate if he is of type $\theta_{2}$ follows from the fact that

$$
\widehat{U}\left(a, \beta^{*}, L, \theta_{2}\right)=a\left(\frac{\left(\beta^{*}\right)^{2} s_{2}^{2}}{2}+L\right)+(1-a)\left(\frac{\left(\beta^{*}\right)^{2} s_{0}^{2}}{2}\right)
$$

and (B41) implies $\frac{\partial}{\partial a} \widehat{U}\left(a, \beta^{*}, L, \theta_{2}\right)>0$ for all $a \in[0,1]$.

Now consider the principal's decision. If $m \in M_{0}$ ("technology is bad"), then the condition for the principal not to deviate can be written:

$$
\pi_{0}\left(\beta_{0}\right) \geq \pi_{1}\left(\beta_{0}\right)
$$

which is true by Remark 3. If $m \in M_{1}$ ("technology is good"), then the condition for the principal not to deviate can be written:

$$
\left(\frac{\rho_{2}}{\rho_{2}+\rho_{3}}\right) \pi_{2}\left(\beta_{0}\right)+\left(\frac{\rho_{3}}{\rho_{2}+\rho_{3}}\right) \pi_{3}\left(\beta_{0}\right) \geq \pi_{0}\left(\beta_{0}\right)
$$

where there is no $L$ on the left hand side since we, rather than the principal, pay the lump-sum bonus. Since $\pi_{2}\left(\beta_{0}\right)>\pi_{0}\left(\beta_{0}\right)$ by Remark 1 and $\pi_{3}\left(\beta_{0}\right)>\pi_{0}\left(\beta_{0}\right)$ is true by Remark 2 , the left-hand side is a weighted average of two quantities greater than $\pi_{0}\left(\beta_{0}\right)$ and (B43) holds. Hence neither the agent nor the principal has an incentive to deviate. 


\section{B.5 Miscellaneous Proofs}

The following remarks establish several properties of the profit functions defined in (B3).

Remark 1. Given the definition of $\pi_{i}(\cdot)$ in (B3), of $\beta_{i}$ in (B4), and condition (B7a), we have:

$$
\pi_{2}(\beta)-\pi_{0}(\beta) \begin{cases}<0 & \text { if } 0<\beta<\hat{\beta}_{2} \\ =0 & \text { if } \beta=\hat{\beta}_{2} \\ >0 & \text { if } \beta>\hat{\beta}_{2}\end{cases}
$$

where $0<\hat{\beta}_{2}<\beta_{0}$.

Proof. From (B3), we can write:

$$
\pi_{2}(\beta)-\pi_{0}(\beta)=\left(s_{0}^{2}-s_{2}^{2}\right)(\beta-\Omega)^{2}-\Omega^{2}\left(s_{0}^{2}-s_{2}^{2}\right)-F
$$

where $\Omega=\frac{s_{0}^{2} \beta_{0}-s_{2}^{2} \beta_{2}}{s_{0}^{2}-s_{2}^{2}}$. This defines a convex parabola with vertex at $\left(\Omega,-\Omega^{2}\left(s_{0}^{2}-s_{2}^{2}\right)-F\right)$. Setting $\pi_{2}(\beta)-\pi_{0}(\beta)=0$ gives two critical values of $\beta$. Since we are requiring $\beta>0$, we ignore the negative root. The positive root defines the value of $\hat{\beta}_{2}: \hat{\beta}_{2}=\Omega+\sqrt{\Omega^{2}+\frac{F}{s_{0}{ }^{2}-s_{2}{ }^{2}}}$. Condition (B7a) requires that $\pi_{2}\left(\beta_{0}\right)-\pi_{0}\left(\beta_{0}\right)>0$ and hence that $\beta_{0}>\hat{\beta}_{2}$.

Remark 2. Given the definition of $\pi_{i}(\cdot)$ in (B3), of $\beta_{i}$ in (B4), and condition (B7b), we have:

$$
\pi_{3}(\beta)-\pi_{0}(\beta) \begin{cases}<0 & \text { if } \beta<\hat{\beta}_{3} \\ =0 & \text { if } \beta=\hat{\beta}_{3} \\ >0 & \text { if } \hat{\beta}_{3}<\beta<\hat{\hat{\beta}}_{3} \\ =0 & \text { if } \beta=\hat{\hat{\beta}}_{3} \\ <0 & \text { if } \beta>\hat{\hat{\beta}}_{3}\end{cases}
$$

where $0<\hat{\beta}_{3}<\beta_{0}<\beta_{2}<\hat{\hat{\beta}}_{3}<2 \beta_{0}$.

Proof. From (B3), we can write:

$$
\pi_{3}(\beta)-\pi_{0}(\beta)=-\left(s_{3}^{2}-s_{0}^{2}\right)\left(\beta-\beta_{0}\right)^{2}+\beta_{0}^{2}\left(s_{3}^{2}-s_{0}^{2}\right)-F
$$

This defines a concave parabola with vertex at $\left(\beta_{0}, \beta_{0}^{2}\left(s_{3}^{2}-s_{0}^{2}\right)-F\right)$. Condition (B7b) implies that $\pi_{3}\left(\beta_{0}\right)>\pi_{0}\left(\beta_{0}\right)$, since $\pi_{3}(\beta)-\pi_{0}(\beta)$ is decreasing over $\left(\beta_{0}, \beta_{2}\right]$, and this in turn implies $\beta_{0}^{2}\left(s_{3}{ }^{2}-s_{0}{ }^{2}\right)-F>0$. Setting $\pi_{3}(\beta)-\pi_{0}(\beta)=0$ defines the values of the roots: $\hat{\beta}_{3}=\beta_{0}-$ $\sqrt{\beta_{0}^{2}-\frac{F}{s_{3}^{2}-s_{0}^{2}}}, \hat{\hat{\beta}}_{3}=\beta_{0}+\sqrt{\beta_{0}^{2}-\frac{F}{s_{3}{ }^{2}-s_{0}^{2}}}$. The facts that $0<\hat{\beta}_{3}<\beta_{0}<\hat{\hat{\beta}}_{3}<2 \beta_{0}$ follow directly from the expressions for $\hat{\beta}_{3}$ and $\hat{\hat{\beta}}_{3}$. Condition $(\mathrm{B} 7 \mathrm{~b})$ requires that $\pi_{3}\left(\beta_{2}\right)-\pi_{0}\left(\beta_{2}\right)>0$ and hence that $\beta_{2}<\hat{\hat{\beta}}_{3}$.

Remark 3. Given the definition of $\pi_{i}(\cdot)$ in (B3) and of $\beta_{i}$ in (B4), we have:

$$
\pi_{1}(\beta)-\pi_{0}(\beta) \begin{cases}<0 & \text { if } 0<\beta<\hat{\hat{\beta}}_{1} \\ =0 & \text { if } \beta=\hat{\hat{\beta}}_{1} \\ >0 & \text { if } \beta>\hat{\hat{\beta}}_{1}\end{cases}
$$


where $\hat{\hat{\beta}}_{1}>2 \beta_{0}>\hat{\hat{\beta}}_{3}$.

Proof. From (B3), we can write

$$
\pi_{1}(\beta)-\pi_{0}(\beta)=\left(s_{0}^{2}-s_{1}^{2}\right)\left(\beta-\beta_{0}\right)^{2}-\left(s_{0}^{2}-s_{1}^{2}\right) \beta_{0}^{2}-F
$$

which is a convex parabola with roots $\hat{\beta}_{1}=\beta_{0}-\sqrt{\beta_{0}^{2}+\frac{F}{s_{0}^{2}-s_{1}^{2}}}<0$ and $\hat{\beta}_{1}=\beta_{0}+\sqrt{\beta_{0}^{2}+\frac{F}{s_{0}^{2}-s_{1}^{2}}}$. Note that $\hat{\beta}_{1}<0$. The fact that $\hat{\hat{\beta}}_{1}>2 \beta_{0}$ follows immediately from the expression for $\hat{\hat{\beta}}_{1}$. The fact that $2 \beta_{0}>\hat{\beta}_{3}$ is from Remark 2 .

Remark 4. Conditions ( $\left.B^{r} / a\right),\left(B^{7} b\right)$ and $\left(B^{7} c\right)$ are compatible with each other.

Intuitively, it is straightforward to see that condition (B7a) can be satisfied as long as $F$ is not too large and the slower speed $s_{2}$ is sufficiently compensated by the lower costs $c_{2}$ (compared to $s_{0}$ and $c_{0}$ ). Condition (B7b) is satisfied as long as $F$ is not too large and $s_{3}$ is sufficiently faster than $s_{0}$. Condition (B7c) is satisfied as long as the bad technology is sufficiently likely, i.e. $\rho_{1}$ is large, and the bad technology is sufficiently bad relative to the existing technology, i.e. $s_{1}$ is sufficiently low.

Proof. There are three conditions:

Condition (B7a)

$$
\pi_{2}\left(\beta_{0}\right)>\pi_{0}\left(\beta_{0}\right)
$$

which can be rewritten in terms of primitives as:

$$
s_{2}^{2}\left(\frac{p-c_{0}}{2}\right)\left(p-\left(\frac{p-c_{0}}{2}\right)-c_{2}\right)-F>s_{0}^{2}\left(\frac{p-c_{0}}{2}\right)\left(p-\left(\frac{p-c_{0}}{2}\right)-c_{0}\right)
$$

Condition (B7b)

$$
\pi_{3}\left(\beta_{2}\right)>\pi_{0}\left(\beta_{2}\right)
$$

which can be rewritten in terms of primitives as:

$$
s_{3}^{2}\left(\frac{p-c_{2}}{2}\right)\left(p-\left(\frac{p-c_{2}}{2}\right)-c_{0}\right)-F>s_{0}^{2}\left(\frac{p-c_{2}}{2}\right)\left(p-\left(\frac{p-c_{2}}{2}\right)-c_{0}\right)
$$

Condition (B7c)

$$
\pi_{0}\left(\beta_{0}\right)>\widetilde{\pi}(\widetilde{\beta})
$$

which can be rewritten in terms of primitives as:

$$
s_{0}^{2}\left(\frac{p-c_{0}}{2}\right)\left(p-\left(\frac{p-c_{0}}{2}\right)-c_{0}\right)>\left(\sum_{i=1}^{3} \rho_{i} s_{i}^{2}\right)\left(\sum_{i=1}^{3} \frac{\rho_{i} s_{i}^{2}}{\sum_{1}^{3} \rho_{i} s_{i}^{2}}\left(\frac{p-c_{i}}{2}\right)\right)^{2}-F
$$

To see that the three can be satisfied simultaneously, consider the following chain:

First, pick a $s_{0}, c_{0}$ and $p$ such that $p-\left(\frac{p-c_{0}}{2}\right)-c_{0}$ is positive (i.e. so that the original technology is profitable at $\beta_{0}$ ).

Second, manipulate $s_{2}, c_{2}, s_{3}$ and $F$ to ensure both (B7a) and (B7b) are simultaneously satisfied (i.e. lower $s_{2}$ below $s_{0}$, lower $c_{2}$ below $c_{0}$, and raise $s_{3}$ above $s_{0}$ ). This is always possible as in the limit when $F \rightarrow 0$ and $s_{2} \rightarrow s_{0}$ (B7a) will be strictly satisfied, and in the limit when $F \rightarrow 0$ (B7b) will be strictly satisfied. 
Third, still holding fixed $s_{0}, c_{0}$ and $p$ at the values from step 1, and holding fixed $s_{2}, c_{2}, s_{3}$ and $F$ at a combination that satisfies (B7a) and (B7b) from step 2, we can raise $\rho_{1}$ (and correspondingly lower $\rho_{2}$ and $\rho_{3}$ since $\left.\sum_{i=1}^{3} \rho_{i}=1\right)$ and lower $s_{1}$ below $s_{0}$ to ensure that (B7c) holds. This will always be possible since in the limit when $\rho_{1} \rightarrow 1$ and $s_{1} \rightarrow 0$ (B7c) will be strictly satisfied.

Remark 5. Given the definition of $\pi_{i}(\cdot)$ in (B3) and conditions (B $\left.{ }^{r} b\right)$ and $\left(B^{r}\right.$ c), we have:

$$
Z(\beta) \equiv\left[\frac{\rho_{1}}{\rho_{1}+\rho_{2}} \pi_{1}(\beta)+\frac{\rho_{2}}{\rho_{1}+\rho_{2}} \pi_{2}(\beta)\right]-\pi_{0}(\beta) \begin{cases}<0 & \text { if } \hat{\beta}_{2} \leq \beta<\bar{\beta} \\ =0 & \text { if } \beta=\bar{\beta} \\ >0 & \text { if } \beta>\bar{\beta}\end{cases}
$$

for some $\bar{\beta}>\beta_{0}$.

Proof. We first consider $\beta=\beta_{0}$. By condition $(\mathrm{B} 7 \mathrm{c}), \pi_{0}\left(\beta_{0}\right)>\widetilde{\pi}(\widetilde{\beta})$. Since $\widetilde{\beta}$ is the optimal choice if the principal bases her decision only on her priors, it must be the case that $\widetilde{\pi}(\widetilde{\beta}) \geq \widetilde{\pi}\left(\beta_{0}\right)$. Hence $\pi_{0}\left(\beta_{0}\right)>\widetilde{\pi}\left(\beta_{0}\right)$. This in turn implies:

$$
\begin{aligned}
\pi_{0}\left(\beta_{0}\right) & >\rho_{1} \pi_{1}\left(\beta_{0}\right)+\rho_{2} \pi_{2}\left(\beta_{0}\right)+\rho_{3} \pi_{3}\left(\beta_{0}\right) \\
\pi_{0}\left(\beta_{0}\right)-\rho_{3} \pi_{3}\left(\beta_{0}\right) & >\rho_{1} \pi_{1}\left(\beta_{0}\right)+\rho_{2} \pi_{2}\left(\beta_{0}\right) \\
\pi_{0}\left(\beta_{0}\right)-\rho_{3} \pi_{0}\left(\beta_{0}\right) & >\rho_{1} \pi_{1}\left(\beta_{0}\right)+\rho_{2} \pi_{2}\left(\beta_{0}\right) \\
\left(\rho_{1}+\rho_{2}\right) \pi_{0}\left(\beta_{0}\right) & >\rho_{1} \pi_{1}\left(\beta_{0}\right)+\rho_{2} \pi_{2}\left(\beta_{0}\right) \\
0 & >\left[\frac{\rho_{1}}{\rho_{1}+\rho_{2}} \pi_{1}\left(\beta_{0}\right)+\frac{\rho_{2}}{\rho_{1}+\rho_{2}} \pi_{2}\left(\beta_{0}\right)\right]-\pi_{0}\left(\beta_{0}\right)=Z\left(\beta_{0}\right)
\end{aligned}
$$

where the third inequality follows from the fact that $\pi_{3}\left(\beta_{0}\right)>\pi_{0}\left(\beta_{0}\right)$ (from condition (B7b)).

Now consider $\beta \in\left[\hat{\beta}_{2}, \beta_{0}\right)$. Note that:

$$
Z(\beta)=\frac{\rho_{1}\left[\pi_{1}(\beta)-\pi_{0}(\beta)\right]+\rho_{2}\left[\pi_{2}(\beta)-\pi_{0}(\beta)\right]}{\rho_{1}+\rho_{2}}
$$

By Remark 3, $\pi_{1}(\beta)-\pi_{0}(\beta)<0$ in this region. From (B44), $\pi_{2}(\beta)-\pi_{0}(\beta)$ is strictly increasing over this region. Hence if $Z\left(\beta_{0}\right)<0$ then must be the case that $Z(\beta)<0$ for all $\beta \in\left[\hat{\beta}_{2}, \beta_{0}\right)$.

Now consider $\beta>\beta_{0}$. Using (B44), (B46) and (B48), we have:

$$
\frac{\partial Z(\beta)}{\partial \beta}=\frac{1}{\rho_{1}+\rho_{2}}\left[2 \rho_{1}\left(s_{0}^{2}-s_{2}^{2}\right)(\beta-\Omega)+2 \rho_{2}\left(s_{0}^{2}-s_{1}^{2}\right)\left(\beta-\beta_{0}\right)\right]
$$

which is strictly positive and increasing in $\beta$ for $\beta>\beta_{0}$ (noting that $\Omega<\hat{\beta}_{2}<\beta_{0}$ ). Since $Z(\beta)$ is negative at $\beta_{0}$ and has strictly positive and increasing slope for $\beta>\beta_{0}$, it takes the value zero at a single point, call it $\bar{\beta}$, and is negative for $\beta \in\left(\beta_{0}, \bar{\beta}\right)$ and positive for $\beta \in(\bar{\beta}, \infty){ }^{22}$

\footnotetext{
${ }^{22}$ To see this, note that $Z(\beta)=\int_{c}^{\beta} Z^{\prime}\left(\beta^{\prime}\right) d \beta^{\prime}+C$ for some finite constants $c$ and $C$. Hence, $\lim _{\beta \rightarrow \infty} Z(\beta)=$ $\int_{c}^{\infty} Z^{\prime}\left(\beta^{\prime}\right) d \beta^{\prime}+C$. Since $Z^{\prime}\left(\beta^{\prime}\right)$ does not go to zero as $\beta^{\prime}$ goes to infinity, $Z(\beta) \longrightarrow \infty$ and so must cross zero.
} 


\section{References}

Aghion, P., And J. Tirole (1997): "Formal and Real Authority in Organizations," Journal of Political Economy, 105(1), 1-29.

Blackwell, D. (1953): "Equivalent Comparisons of Experiments," The Annals of Mathematical Statistics, 24(2), 265-272.

Crawford, V. P., And J. Sobel (1982): "Strategic Information Transmission," Econometrica, 50(6), $1431-1451$.

Halac, M., N. Kartik, And Q. Liu (2016): "Optimal Contracts for Experimentation," Review of Economic Studies, 83, 1040-1091.

Sobel, J. (2013): "Giving and Receiving Advice," in Advances in Economics and Econometrics, ed. by D. Acemoglu, M. Arellano, and E. Dekel. Cambridge University Press. 
Figure B.1: Profit functions for different technology types

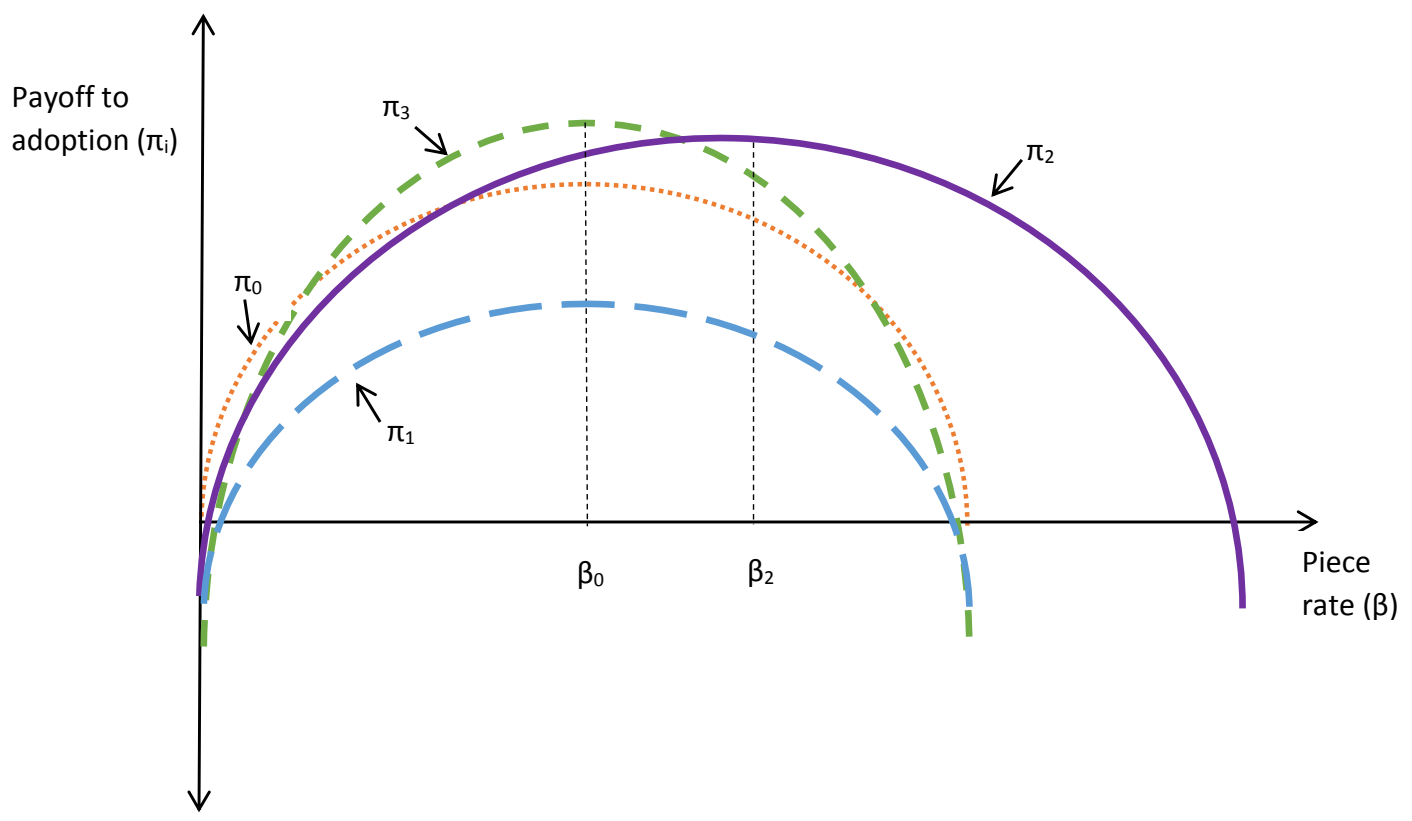

Notes: Figure illustrates the relative positions of the $\pi_{i}(\beta)$ functions (defined by (B3) in Subsection B.1.1) implied by the parameter restrictions (B7a)-(B7c). 